\title{
Annual Report
}

\section{Development of Advanced Electrochemical Emission Spectroscopy for Monitoring Corrosion in Simulated DOE Liquid Waste}

\author{
by \\ Digby D. Macdonald, Brian M. Marx, Sejin Ahn, Julio de Ruiz, Balaji Soundararajan, \\ and Morgan Smith \\ Center for Electrochemical Science and Technology \\ Pennsylvania State University \\ University Park, PA 16802 \\ digby@essc.psu.edu
}

Submitted to

Department of Energy Environmental Science Program

Grant No. DE-FG07-97ER62515 (Project 60219)

June $15^{\text {th }}, 2004$ 


\section{Table of Contents}

Executive Summary ...............................................................................

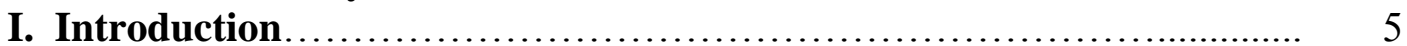

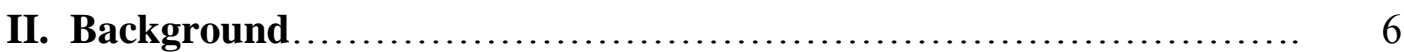

II-1. Development of Corrosion Mechanisms...................... 6

II-2. Theoretical Study of Passivity Breakdown................................. 10

II-3. Crack Propagation Studies................................. 18

II-4. Electrochemical Emission Spectroscopy...................... 23

II-4.1. Generation of EC Noise from General and Localized Corrosion........................................ 23

II-4.2. Computer Simulation of Anodic/Cathodic Activity...... 23

II-4.3. Wavelet Analysis.................................. 25

II-5. Unification of the Deterministic and Statistical Approaches for

Predicting Corrosion Damage............................... 28

III. Research Progress............................................... 29

III-1. Continued Development of the Theory of Passivity ............. 29

III-1.1a,b. Deriving Impedance Equations from PDM......... $\quad 30$

III-1.1c. OPS Work on Iron Oxide Film..................... 47

III-1.2. Obtaining Kinetic Information for Passive Iron via Curve-Fitting................................... 49

III-1.3. Experimental Set-up to Obtain Data for DFA......... 52

III-2. The Role of Chloride Ion in Passivity Breakdown.............. 54

III-2.1. Experimental Set-up................................. 54

III-2.2. Applying Mott-Schottky Analysis and EIS to Study Passivity Breakdown........................ $\quad 55$

III-3. Crack Propagation Studies............................... 66

III-3.1. Experimental Set-up............................ 66

III-3.2. Identification of the Ideal Parameters for Describing Crack Propagation............................... 71

III-3.3. Frequency Domain Analysis for High Solution Concentrations................................. 72

III-3.4. Time Domain Analysis for Low Solution Concentrations................................... 78

III-4. Identifying Type of Corrosion via Electrochemical Emission Spectroscopy ........................................... 88

III-5. Combining the Deterministic and Statistical Approaches For Predicting Localized Corrosion Damage.................... 89

III-5.1. Developing a Theoretical Basis for DFA............. 89

III-5.2. Generalization of PDM to Describe Passivity Breakdown......................................... 98

III-5.3. Developing a Relationship Between the Deterministic and Statistical Methods for Predicting the Progression of Corrosion Damage............... 104

IV. Publications in Peer Reviewed Journals/Conference Proceedings... 121

V. Presentations.................................................... 123

VI. Current Student Status............................................. 123 


\section{EXECUTIVE SUMMARY}

Various forms of general and localized corrosion represent principal threats to the integrity of DOE liquid waste storage tanks. These tanks, which are of a single wall or double wall design, depending upon their age, are fabricated from welded carbon steel and contain a complex waste-form comprising $\mathrm{NaOH}$ and $\mathrm{NaNO}_{3}$, among other chemicals. Because waste leakage can have a profound environmental impact, considerable interest exists in predicting the accumulation of corrosion damage, so as to more effectively schedule maintenance and repair.

The different tasks that are being carried out under the current program are as follows: (1) Theoretical and experimental assessment of general corrosion of iron/steel in borate buffer solutions by using electrochemical impedance spectroscopy (EIS), ellipsometry and XPS techniques; (2) Development of a damage function analysis (DFA) which would help in predicting the accumulation of damage due to pitting corrosion in an environment prototypical of DOE liquid waste systems; (3) Experimental measurementof crack growth rate, acoustic emission signals and coupling currents for fracture in carbon and low alloy steels as functions of mechanical (stress intensity), chemical (conductivity), electrochemical (corrosion potential, ECP), and microstructural (grain size, precipitate size, etc) variables in a systematic manner, with particular attention being focused on the structure of the noise in the current and its correlation with the acoustic emissions; (4) Development of fracture mechanisms for carbon and low alloy steels that are consistent with the crack growth rate, coupling current data and acoustic emissions; (5) Inserting advanced crack growth rate models for SCC into existing deterministic codes for predicting the evolution of corrosion damage in DOE liquid waste storage tanks; (6) Computer simulation of the anodic and cathodic activity on the surface of the steel samples in order to exactly predict the corrosion mechanisms; (7) Wavelet analysis of EC noise data from steel samples undergoing corrosion in an environment similar to that of the high level waste storage containers, to extract data pertaining to general, pitting and stress corrosion processes, from the overall data. 
The Point Defect Model (PDM) is being directly applied as the theoretical assessment method for describing the passive film formed on iron/steels. The PDM is being used to describe general corrosion in the passive region of iron. However, previous work suggests that pit formation is due to the coalescence of cation vacancies at the metal/film interface. This previous work suggests that once the critical vacancy density is reached, the film ruptures to form a pit. Based upon the kinetic parameters derived for the general corrosion case, two parameters relating to the cation vacancy coalescence can be calculated. These two parameters can then be applied to predict the transition from general to pitting corrosion for iron/mild steels. If this view proves to be the case, this information can have a profound effect on the direction of future studies involving the onset of pitting corrosion.

The work has yielded a number of important findings, including an unequivocal demonstration of the role of chloride ion in passivity breakdown on nickel in terms of cation vacancy generation within the passive film, the first detection and characterization of individual micro fracture events in stress corrosion cracking, and the determination of kinetic parameters for the generation and annihilation of point defects in the passive film on iron. The existence of coupling between the internal crack environment and the external cathodic environment, as predicted by the coupled environment fracture model (CEFM), has also been indisputably established for the AISI 4340/ $\mathrm{NaOH}$ system. It is evident from the studies that analysis of coupling current noise is a very sensitive tool for studying the crack tip processes in relation to the chemical, mechanical, electrochemical and microstructural properties of the system. Experiments are currently being carried out to explore these crack tip processes by simultaneous measurement of the acoustic activity at the crack tip in an effort to validate the coupling current data. These latter data are now being used to deterministically predict the accumulation of general and localized corrosion damage on carbon in prototypical DOE liquid waste storage tanks. Computer simulation of the cathodic and anodic activity on the steel surfaces is also being carried out in an effort to simulate the actual corrosion process. Wavelet analysis of the coupling current data promises to be a useful tool to differentiate between the different corrosion mechanisms. Hence, wavelet analysis of the coupling current data from the DOE waste containers is also being carried out to extract data pertaining to general, pitting and stress 
corrosion processes, from the overall data which is bound to contain noise fluctuations due to any or all of the above mentioned processes

\section{INTRODUCTION}

DOE currently stores about 253 million liters of high-level nuclear waste at the Hanford Reservation in Washington. The waste consists of a concentrated solution of $\mathrm{NaOH}, \mathrm{NaNO}_{3}, \mathrm{NaNO}_{2}$, and $\mathrm{NaCl}$, with other species such as $\mathrm{NaSO}_{4}, \mathrm{NaHPO}_{4}, \mathrm{NaAlO}_{2}$, and $\mathrm{NaF}$ also being present. Additionally, various radioactive species and "sludge", comprising a mixture of precipitated salts, are also present in the system. The temperature of the waste is typically $95^{\circ} \mathrm{C}-97^{\circ} \mathrm{C}$ and the $\mathrm{pH}\left(25^{\circ} \mathrm{C}\right)$ is greater than 12 . The waste is stored in a variety of single walled and doubled wall tanks manufactured from ASTM A537-Cl. 1, ASTM A515-Gr. 60, or ASTM A516-Gr. 60 steel. The single walled tanks were not stress relieved after welding, and hence contain weld heat-affected zones (HAZs) that are partially martensitic. These HAZs are susceptible to stress corrosion cracking (SCC) and/or hydrogen-induced fracture (HIC). Some of the single walled tanks are suspected to be leaking due to corrosion-induced penetrations of the walls.

The grades of steel used for the storage of DOE waste exhibit good corrosion resistance when in contact with alkaline solutions at temperatures below $100^{\circ} \mathrm{C}$. Of particular importance is the presence of chloride and sulfate ions in the waste, because these species are known to induce pitting corrosion on carbon and low alloy steels, amongst other alloys. Accordingly, general corrosion, pitting corrosion, and stress corrosion cracking are the most important degradation mechanisms. Although the general corrosion rate of iron in caustic environments is very low $\left(<0.1 \mathrm{~mm} / \mathrm{yr}\right.$ at $\left.100^{\circ} \mathrm{C}\right)$, the long storage times $(100+$ years) makes possible the accumulation of significant general corrosion damage. Exacerbating the corrosion problem is the presence of water radiolysis products, such as $\mathrm{H}_{2} \mathrm{O}_{2}, \mathrm{O}_{2}$, and $\mathrm{H}_{2}$, which may alter the corrosion potential and lead to the onset of specific failure processes, such as caustic and nitrate cracking.

One of the major problems in ensuring tank integrity is the detection and monitoring of various forms of corrosion. In the case of general corrosion, the problem stems from the inherently high corrosion resistance of carbon steel in alkaline environments, which implies that the corrosion current density is low (typically $<1$ 
$\mu \mathrm{A} / \mathrm{cm}^{2}$ ). Because iron is highly passive, the noise in the current and potential, due to fluctuations in the local anodes and cathodes on the surface, is also very small (typically a few nano amperes and milli volts, respectively), making their accurate measurement difficult. Accordingly, any monitoring technique that is devised to monitor general corrosion remotely faces the challenge of transmitting very small signals from the sensors to the recording points without significant modification due to external interference (for example). On the other hand, localized corrosion processes, such as pitting corrosion and stress corrosion cracking, produce significant noise in both the potential and current, but because they are highly localized it is difficult, if not impossible, to predict a priori the exact locations at which they will occur and hence should be monitored. Accordingly, monitoring of these phenomena is best carried out using surveillance specimens that are configured to induce specific forms of localized corrosion.

After acquiring the potential and current noise fluctuations from the carbon steel containers, it is important to have suitable methods to extract data pertaining to general, pitting and stress corrosion processes from the overall data which contains noise fluctuations due to any or all of the above mentioned processes. Suitable methods are being developed to solve this problem.

Additionally, it is possible that at any given time the dominant corrosion mechanisms can be predicted by a suitably constructed computer simulation. Such a simulation would involve modeling the anodic and cathodic activity on the surface of the specimens and manipulating the rules governing the simulation until the EC noise resembled that of the experimental data set. Accordingly, simulation work is also currently being carried out.

\section{BACKGROUND}

\section{II-1. Development of Corrosion Mechanisms}

General Corrosion occurs by the direct dissolution of a metal in the active state or by the dissolution of the passive oxide film in the passive state. In the latter case, which is the appropriate scenario for iron in DOE liquid waste, passive film dissolution is compensated for by growth of the oxide into the metal substrate, so that the corrosion rate is ultimately determined by the rate of dissolution of the oxide film and by the 
transmission of cations through the film. Accordingly, any model that is developed to predict the accumulation of damage due to general corrosion must address both the film dissolution and film formation processes that occur at the film/solution interface and at the metal/film interface, respectively. This issue has been addressed during the current program with the derivation of a new rate law for the growth of a passive film on a metal surface. The new law is based on the Point Defect Model (PDM) [1] for the growth and breakdown of passive films. The derivation of this rate law was shown in an earlier report and is not shown here. It should just be pointed out that this rate law depends on knowing the kinetic parameters for the reactions depicted by the PDM. Therefore, the focus of the current work on general corrosion is to obtain these kinetic parameters.

The goal of this portion of the project is to provide an assessment of the general and local corrosion of iron/steel. Knowledge of the kinetic parameters will lead to a much greater understanding of the general corrosion process occurring on iron/steels and will ultimately allow for much more accurate failure predictions to be made. Damage Function Analysis (DFA) [2] will be implemented to predict damage accumulation due to pitting corrosion. Eventually, the kinetic parameters derived by the PDM will be incorporated into DFA. This combination will yield a deterministic/probabilistic model (based on Extreme Value Statistics) that can be used to predict failure in accordance with the pertinent conditions. Once developed, the PDM/DFA will be applied as a method to analyze data obtained by the monitoring of the electrochemical/environmental conditions such as current noise, $\mathrm{pH}$, aggressive ion concentration, and temperature. The end result will be the formation of an in-depth scientific method capable of monitoring the corrosion of iron/steel structures, containers, and vessels (in contact with solutions) for the purpose of predicting damage/failure.

High purity iron (Alfa Aesar-99.99\%) is being used in this portion of the work to extract the kinetic parameters for use in the PDM. To determine the kinetic parameters, the reactions involved in the corrosion process must be known (or assumed to be the ones involved). Without knowing the reactions occurring, it is impossible to account for the kinetic parameters governing the corrosion process. Extracting kinetic data without knowing/proposing which reactions are occurring would be like providing a destination time/distance without knowing the path being taken to the destination. By using pure 
iron, rather than steel, the number of possible reactions occurring simultaneously at the surface is greatly decreased (like decreasing the number of possible roads that can be taken to reach one's destination). For this reason, high purity iron is used in these experiments. However, some argument may arise as to the validity of applying the kinetic data gathered for iron to steel. This is discussed next.

The iron oxide formed on mild steel is believed to be responsible for the steel's passive characteristics (in the passive region), and not the oxides/chemical compounds formed by the other additives in the steel (C, Mg, Mn, etc.) [5]. Therefore, it is much simpler to account for the reactions occurring at the surface of the pure iron rather than the multitude of reactions possible on a steel surface. Although it is likely that the other elements in the steel could be involved in reactions occurring at the surface, their influence is considered to be very minor due to their very low concentrations. From a scientific point of view, it is beneficial to use a high purity material to account much more precisely for the kinetic parameters of the relevant reactions. After collecting the kinetic parameters for iron, some experiments will be carried out on mild steel to ensure that the kinetic parameters derived for the iron are valid for steel. Previous fundamental work on the PDM lists the reactions assumed to be happening at the iron/oxide/solution interface [3].

Despite the fact that the fundamental reactions are known, a complication arises due to the fact that iron forms a double oxide layer comprised of an inner layer of $\mathrm{Fe}_{3} \mathrm{O}_{4}$ (magnetite) and an outer precipitation layer of $\mathrm{Fe}_{2} \mathrm{O}_{3}$ (hematite). It is widely accepted that the inner magnetite layer is responsible for the passive behavior of iron. However, the outer layer of hematite obstructs the inner layer from experimental observation.

Early work on this project reveals a method for removing the outer precipitation layer formed on iron, enabling the inner layer to be studied directly $[3,4]$. It has been shown that the addition of EDTA (chelating agent) to a borate buffer solution prevents the outer precipitation layer (hematite, $\mathrm{Fe}_{2} \mathrm{O}_{3}$ ) from forming on iron in solution. By removing the precipitation layer, the inner $\mathrm{Fe}_{3} \mathrm{O}_{4}$ (magnetite) can be studied directly. The early fundamental work on the PDM reactions for iron and the work on the removal of the precipitation layer made it possible to experimental obtain kinetic parameters making it possible to build a comprehensive database of kinetic parameters as functions of 
temperature, potential, and $\mathrm{pH}$. This database will allow us to analyze electrochemical data by the PDM and make predictions concerning damage/failure of iron/steels for various conditions.

The next step is to derive the equations for the impedance and thickness of the iron oxide film $\left(\mathrm{Fe}_{3} \mathrm{O}_{4}\right)$ as described by the PDM. Once the equations are derived, experiments can be performed to measure the impedance and the film thickness. Figure II-1.1 shows the experimental setup in which a Solartron 1250 Frequency Response Analyzer (FRA) is being used for Electrochemical Impedance Spectroscopy (EIS), a Solartron 1286 potentiostat is being used for potentiostatic control, and a Sopra scanning wavelength ellipsometer is being used to measure the film thickness. DataFit software will be used to fit the impedance equation to the data. Once a reasonable fit is obtained, the kinetic parameters can be extracted from the simulation. These parameters will then be used to predict the impedance of films as functions of temperature, $\mathrm{pH}$, and potential. Further experiments will be performed to verify these predictions.

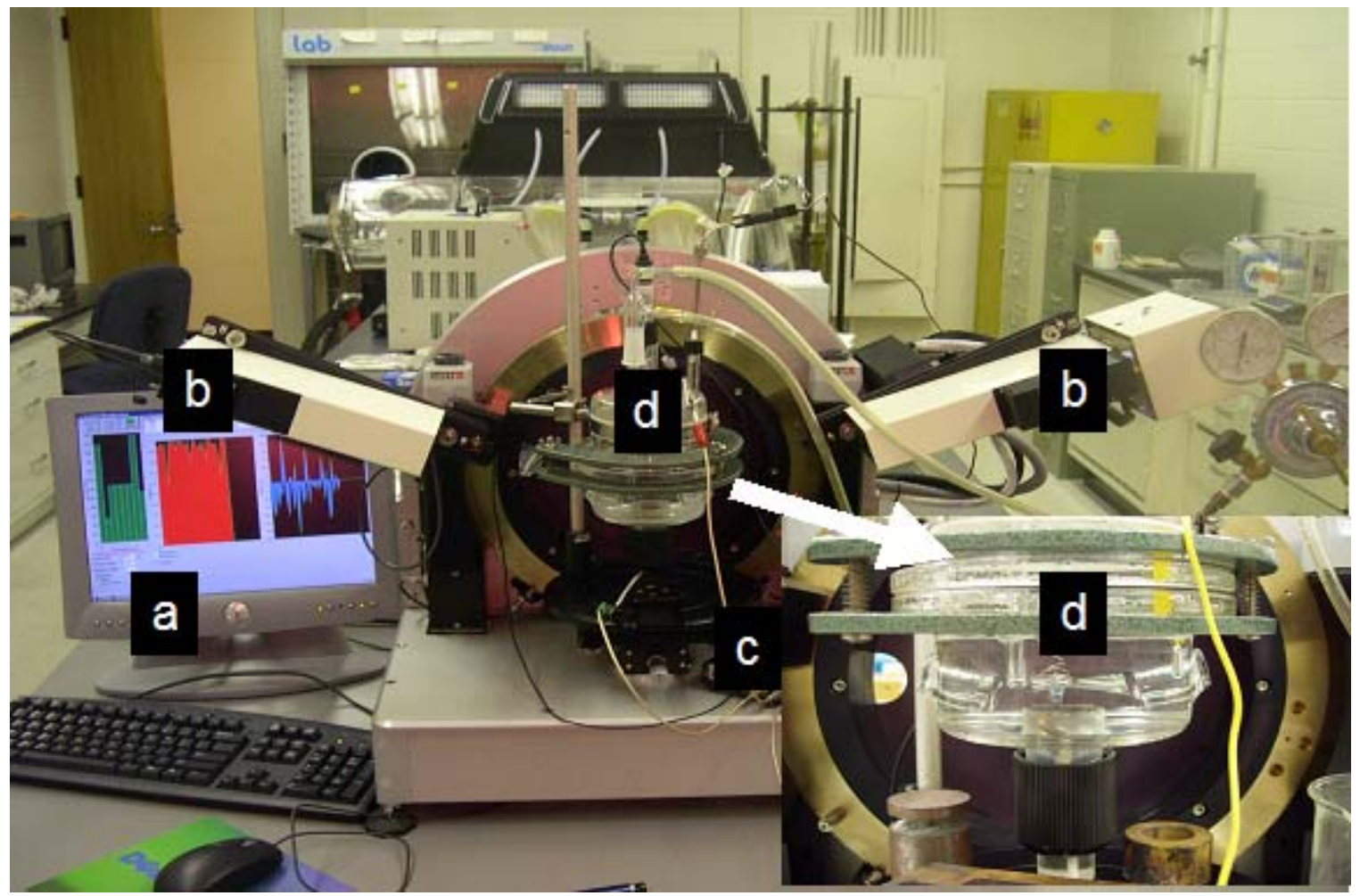

Figure II-1.1 Picture of the spectroscopic ellipsometer and associated systems. The PC used to collect the ellipsometric and impedance data (a). Two arms of ellipsometer-used to detect the changes in reflected 
light (b). Potentiostat/Frequency Response Analyzer (FRA) for controlling the potential at the working electrode and measuring AC impedance (c). The cell containing the electrochemical set-up (d).

This report breaks the work into sections under the research progress heading. Each section describes the tasks being performed, the immediate goals of each, and what work has been completed. For the general/pitting corrosion work, the first section discusses the experimental methodology used to obtain impedance and ellipsometry data that will be analyzed to extract the kinetic parameters required by the PDM for making predictions. Also in this section is a brief description of some XPS work that has been performed to quantify the relative amounts of the iron species in the film. The final section describes the experimental setup being developed to obtain pitting data for use in DFA.

\section{II-1. References}

1. D.D. Macdonald, M. Uriquidi-Macdonald, J. of Electrochemical Society, 137, 2395 (1990).

2. G. Engalhardt, D.D. Macdonald, Corrosion, 54, 469 (1998).

3. J. Liu, D.D. Macdonald, J. of Electrochemical Society, 148, B425 (2001).

4. E. Sikora, D.D. Macdonald, J. of Electrochemical Society, 147, 4087 (2000).

5. A. Valeria, C.M.A. Brett, Electrochimica Acta, 47, 2081 (2002).

\section{II-2. Theoretical Study of Passivity Breakdown}

The passive current density for most reactive transition metals and their alloys (e.g. $\mathrm{Fe}, \mathrm{Ni}, \mathrm{Cr}, \mathrm{Ti}, \mathrm{Zr}$ ) in contact with ambient aqueous environments is of the order of 1 $\mu \mathrm{A} / \mathrm{cm}^{2}$ to $0.01 \mu \mathrm{A} / \mathrm{cm}^{2}$, corresponding to metal penetration rate of about $10 \mu \mathrm{m} /$ year to $0.1 \mu \mathrm{m} /$ year. Penetration rates of this magnitude do not represent a significant threat to the integrity of industrial or infra-structural systems over the design lifetime, except for special cases (e.g. ball bearings) where dimensional stability is critical for proper operation and function. Unfortunately, passive films do not afford complete protection of the substrate metal or alloy, in that certain species (e.g., $\mathrm{Cl}^{-}$) induce passivity breakdown, resulting in various forms of localized corrosion, such as pitting attack and stress 
corrosion cracking. Localized corrosion processes can result in the rapid penetration of a cavity (pit or crack) into the metal substrate, thereby leading to premature failure. Indeed, corrosion in general, of which localized corrosion is a major component, is estimated to cost an industrialized country more than $4 \%$ of the GNP (approximately $\$ 400$ billion for the U.S. in 2002), rendering it one of the most important costs in operating industrial systems. However, the "cost of corrosion" never appears in a corporate balance sheet, so that the cost is largely hidden from the shareholder and consumer alike.

Because of its practical and scientific importance, passivity and passivity breakdown have been studied intensively since the time of Faraday (1830s) and numerous theories and models have been proposed to explain the phenomen [2,6-9]. However, because of the lack of in situ experimental techniques having the required spatial and temporal resolution, few atomic scale details of the processes that occur within the passive film have emerged from these studies. Nevertheless, it is now generally agreed that the barrier oxide layer is a highly defective structure, with the point defects being metal and oxygen vacancies and metal interstitials. The identity of the principal defect depends upon the relative energies of formation upon the system [1].

The PDM provides an analytical description of the growth and breakdown of passive films on reactive metal surfaces [1]. The fundamental basis of the general PDM is illustrated in Figure II-2.1, in which the elementary reactions that lead to the generation and annihilation of point defects at the interfaces (Reactions $1-6$ ), together with film dissolution (Reaction 7), are displayed. Of particular importance in the present discussion are Reactions 4 and 1, which represent the generation and annihilation of cation vacancies at the barrier layer/solution and metal/barrier layer interfaces, respectively, resulting in a flux of cation vacancies across the film as indicated. 


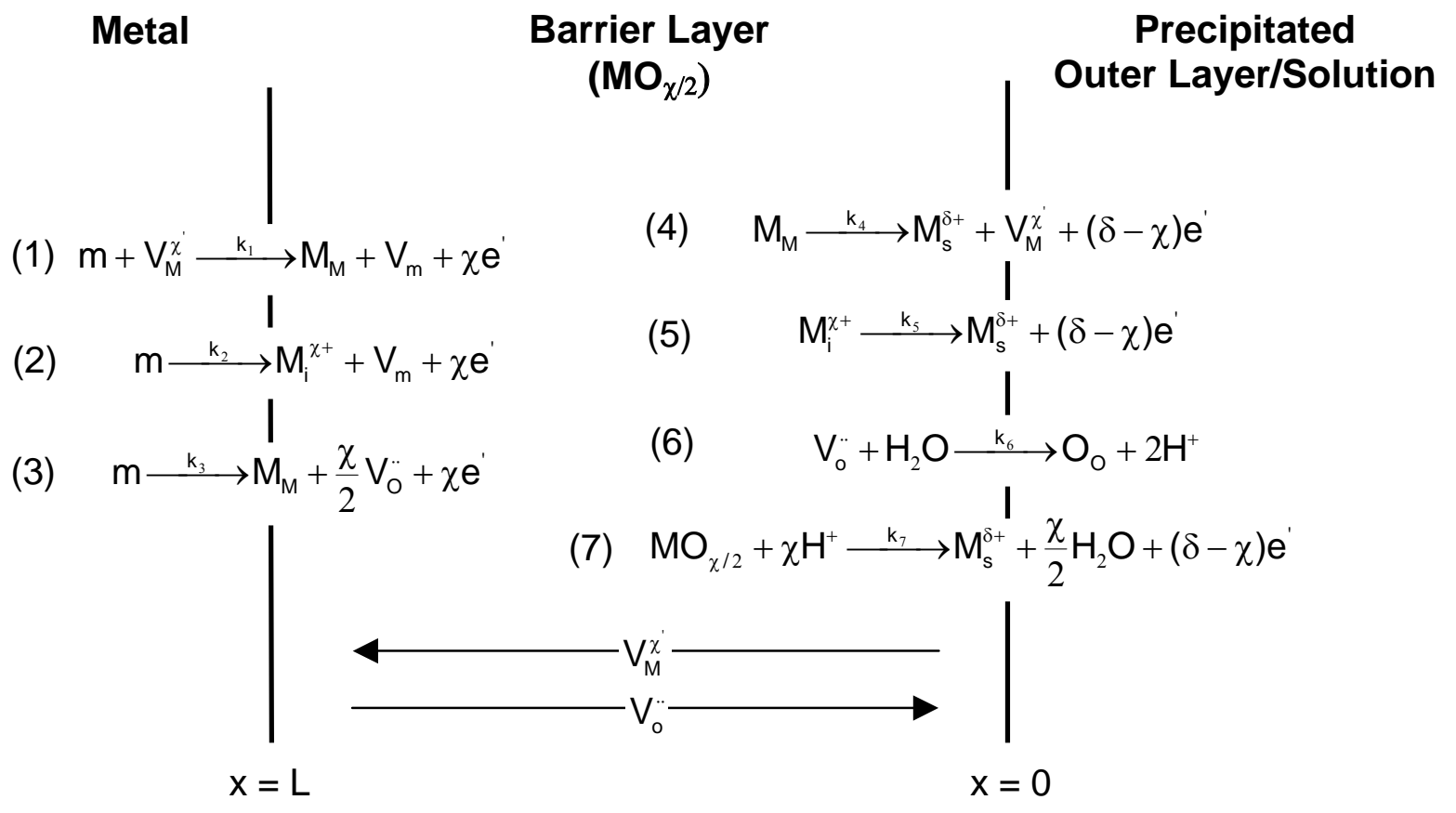

Figure II-2.1 Schematic of physico-chemical processes that occur within a passive film according to the Point Defect Model. $\mathrm{m}=$ metal atom, $V_{M}^{\chi^{\prime}}=$ cation vacancy, $\mathrm{M}_{\mathrm{M}}=$ cation in cation site, $M_{i}^{\chi+}=$ cation in interstitial site, $V_{O}^{\bullet \bullet}=$ anion vacancy, $M_{s}^{\chi+}=$ cation in outer layer/solution, $O_{O}$ = oxygen ion in anion site, $M O_{\chi / 2}=$ stoichiometric barrier layer oxide. Cation vacancies are produced at the film/solution interface, but are consumed at the metal/film interface. Likewise, anion vacancies are formed at the metal/film interface, but are consumed at the film/solution interface. Consequently, the fluxes of cation vacancies and anion vacancies are in the directions indicated [1].

The PDM postulates that, if the cation vacancies arriving at the metal/barrier layer interface cannot be annihilated by Reaction 1, then the vacancies will condense to form a "vacancy condensate" that effectively separates the film from the substrate metal (Figure II-2.2), with vacancy condensation continuing to occur at the periphery, thereby resulting in growth of the blister. Separation of the barrier layer from the metal results in cessation of growth of the barrier layer into the substrate metal or alloy via Reaction 3 , Figure II-2.1 [1]. However, the barrier layer continues to dissolve at the barrier 
layer/solution (outer layer) interface [Reaction 7, Fig. II-2.1], thereby resulting in the local thinning of the barrier layer [Fig. II-2.2(c)]. Thinning continues until the local growth stresses within the barrier layer result in the rupture of the "cap" over the vacancy condensate, finally resulting in a passivity breakdown event, as shown schematically in Figures II-2.2(d) and (e). The nucleus may undergo "prompt repassivation" in which case it represents a "meta stable pitting event, or it may stabilize to form a "stable" pit that will grow to induce macroscopic damage. Eventually, the stable pit will also die via delayed repassivation, which arises for a number of reasons, including the limitation of the resources that are available on the external surfaces and competition between neighboring pits, as outlined previously [1]. The ratio of the probability of formation of a stable pit to the probability of formation of a stable and meta stable pit is known as the survival probability. This quantity is readily measured and is found to have a value of about $10^{-4}$ for pitting on Type 304 stainless steel [10]. Stable pitting is indeed a rare (but very damaging) event. 


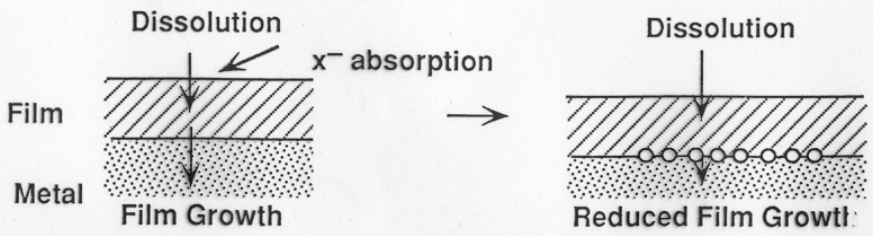

Film Growth

(a) Steady State (b) Vacancy Condensation

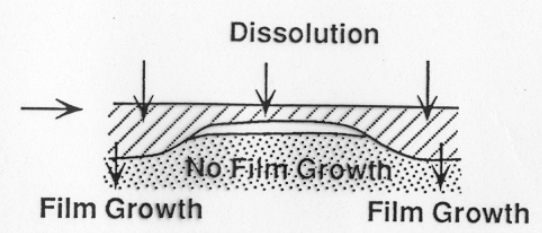

(c) Local Film Detachment

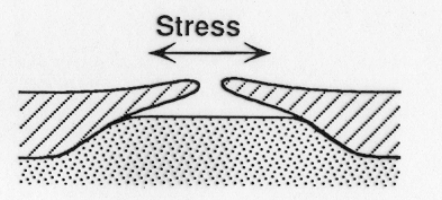

(d) Film Rupture

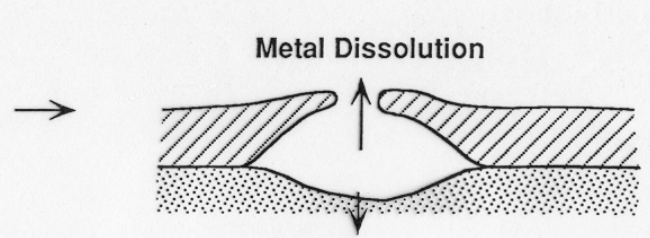

(e) Pit Growth
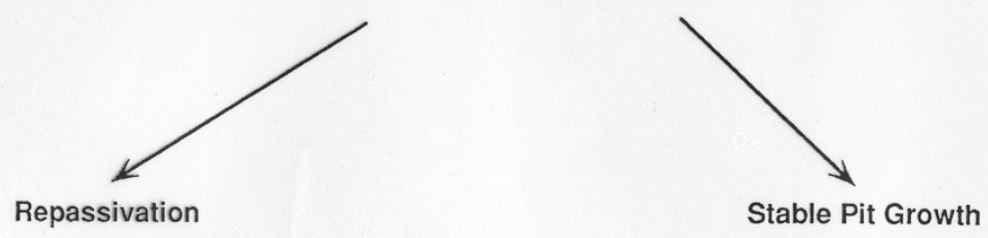

Figure II-2.2 Cartoon outlining various stages of pit nucleation according to the Point Defect Model [1].

This basic picture of the sequence of events in passivity breakdown has received strong experimental support, ranging from the direct observation of blister formation as the precursor to passivity breakdown [11,12], accounting for the photo-inhibition of passivity breakdown [13], to correctly predicting the dependencies of the critical breakdown voltage and induction time on various independent variables \{e.g., $\left[\mathrm{Cl}^{-}\right], \mathrm{pH}$, and potential ( $\mathrm{t}_{\text {ind }}$ only) $\}[1]$, to name but a few of the successful applications of the model. A particularly convincing test of the PDM is afforded by studying the voltage sweep rate dependence of the apparent breakdown voltage, because the quantity that is derived (the areal concentration of vacancies in the condensate) can be compared with the value calculated from structural considerations. Studies of this type that have been performed on nickel [14] and aluminum [15] have found excellent agreement between theory and experiment. Implicit in the model is the concept that aggressive anions, such as $\mathrm{Cl}^{-}$, are able to enhance the flux of cation vacancies through the barrier layer, such that under favorable conditions (voltage, $\mathrm{pH},\left[\mathrm{Cl}^{-}\right]$) vacancy condensation will occur at the 
metal/barrier layer interface and hence passivity breakdown will ensue. Two mechanisms have been proposed for the impact of an aggressive anion on the cation vacancy flux, both of which envision the initial absorption of the anion into a surface oxygen vacancy (Figure II-2.3): (1) Schottky pair formation [16], and (2) cation extraction [1]. Both of these possibilities are represented in Figure II-2.3 and both lead to expressions of identical functional form for the critical breakdown voltage and induction time. Accordingly, it has not been possible to choose between the two possibilities upon the basis of measured dependencies of $V_{c}$ and $t_{\text {ind }}$ on the system independent variables. It is important to note that both mechanisms lead to the regeneration of oxygen vacancies at the barrier layer/solution interface and chloride ion, which is then available to reabsorb into the regenerated oxygen vacancies, to continue the process. This process can only occur above the location of cation vacancy condensation, and hence accounts for the important observation of Bargeron and Givens [11] on passive aluminum that a chloride ring on the surface expands at the periphery of the blister as the blister grows. 


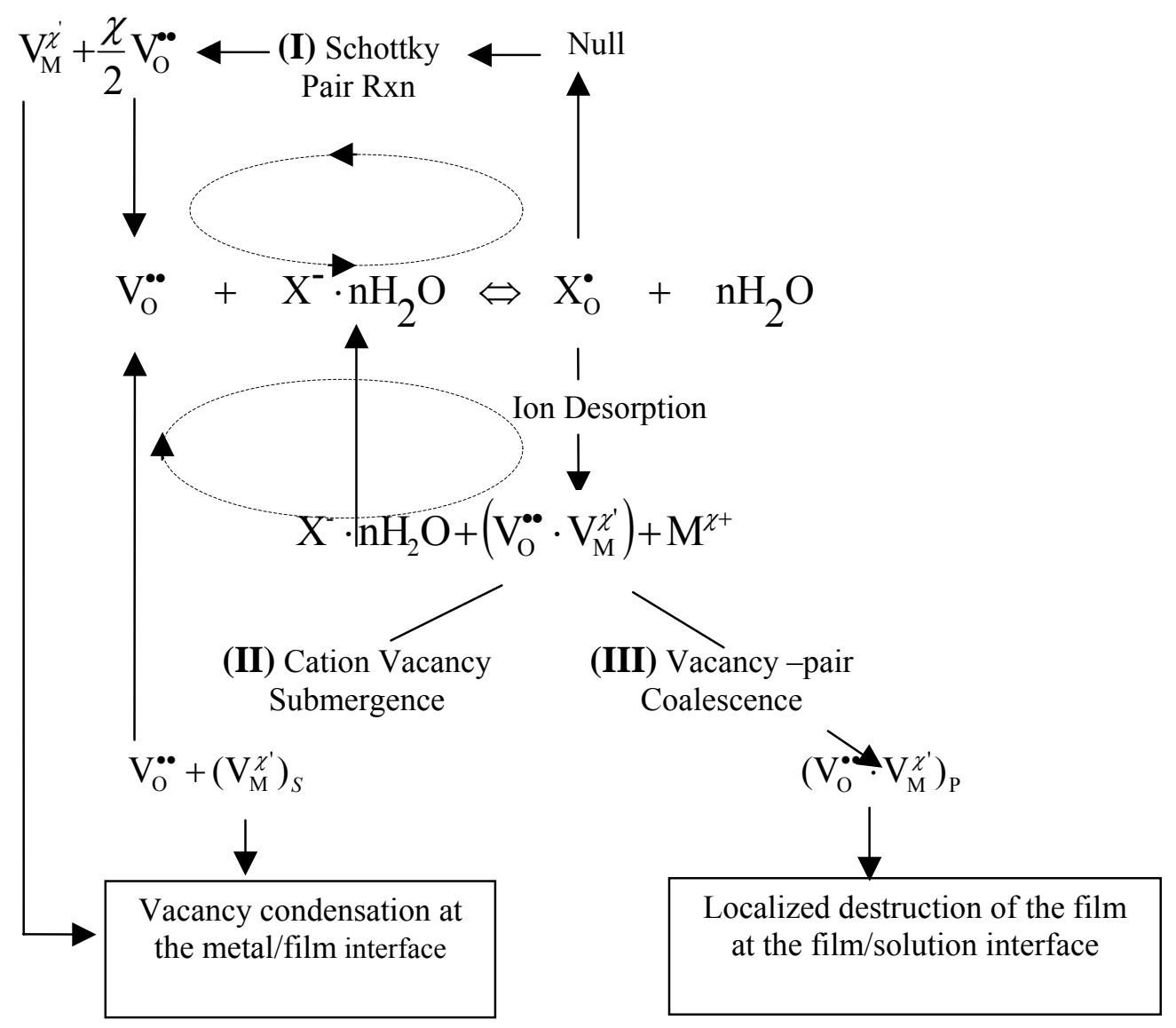

Figure II-2.3 Postulated reactions for cation vacancy generation at the barrier layer/solution interface according to the Point Defect Model [1]. The broken lines indicate the direction of the reactions that are postulated to be responsible for the generation of cation vacancies at the barrier layer/solution interface. Note that the occurrence of the reactions leads to the regeneration of oxygen vacancies and chloride ion and that if vacancy condensation occurs at the periphery of the blister then adsorption of chloride will occur on the outer surface of the barrier layer at the same location, as observed by Bargeron and Givens [10] for passivity breakdown on aluminum.

This section is concerned with experimentally characterizing changes in the vacancy structure of the barrier layer upon exposure of a passive surface to chloride ion and with ascertaining the exact nature of the cation vacancy generation reaction that occurs at the barrier layer/solution interface. Passive nickel was chosen for this study, 
because the passive film on this metal is a p-type semi conductor, indicating that the majority defect is the cation vacancy. Thus, any impact that chloride ion has on the concentration of the cation vacancy in, and the flux across, the barrier layer, increase of which are postulated in the PDM to be responsible for passivity breakdown, should be readily detected using Mott-Schottky analysis. Specifically, the work was designed to detect changes in cation vacancy concentration in the passive film upon absorption of chloride into surface oxygen vacancies and to explore the nature of any cation vacancy generation process that may occur at the barrier layer/solution interface.

In this communication, we assess the impact that chloride ion, which is known to induce passivity breakdown in many metals and alloys, has on the electronic and point defect structures of the passive film on nickel. The principal findings are as follows:

- We show by Mott-Schottky analysis that, as the concentration of $\mathrm{Cl}^{-}$in $\mathrm{pH} 8.5$ borate buffer solution increases, the concentration of metal vacancies $\left(V_{M}^{\chi^{\prime}}\right)$ in the passive film on $\mathrm{Ni}$ also increases.

- The addition of $\mathrm{Cl}^{-}$to $\mathrm{pH} 8.5$ borate buffer solution after passivity has been established in the absence of $\mathrm{Cl}^{-}$also results in an increase in the concentration of metal vacancies $\left(V_{N i}^{\chi^{\prime}}\right)$ in the passive film.

- Finally, by using electrochemical impedance spectroscopy to interrogate the point defect generation and annihilation reactions that occur at the metal/film and film/solution interfaces, we show that the observed increase in cation vacancy concentration in the passive film is due to chloride catalyzed ejection of cations from the film/solution interface.

These findings are inconsistent with chloride-catalyzed film dissolution and chloride penetration mechanisms for passivity breakdown, but they are consistent with the Point Defect Model.

\section{II-2 References}


1. D. D. Macdonald, Pure Appl. Chem., 71, 6, 951 (1999).

2. H. H. Uhlig, Passivity of Metals (R. P. Frankenthal, J. Kuger, eds), p.1. The Electrochemical Soc., Princeton, NJ (1978).

3. K. Ismail, E. Sikora, and D. D. Macdonald, J. Electrochem. Soc., 145, 3141 (1998).

4. E. Sikora and D. D. Macdonald, J. Electrochem. Soc., 147(11), 4087 (2000).

5. J. Liu and D. D. Macdonald, J. Electrochem. Soc., 148, B425 (2001).

6. R. P. Frankenthal and J. Kruger (eds.), Passivity of Metals, The Electrochemical Society, Princeton, NJ (1978).

7. M. Fromont (ed.) Passivity of Metals and Semiconductors, Elsevier Scientific, Amsterdam, (1983).

8. D. D. Macdonald, J. Electrochem. Soc., 139, 3434 (1992).

9. N. Sato, Passivity of Metals (R. P. Frankenthal, J. Kuger, eds), p.29. The Electrochemical Soc., Princeton, NJ (1978).

10. D. Williams, J Stewart and P. H. Balkwill, Corr. Sci., 36, 1213 (1994).

11. C. B. Bargeron and R. B. Givens, Corrosion, 36, 618 (1980).

12. D. J. Ellerbrock and D. D. Macdonald. Materials Science Forum, 185-188, 927 (1995).

\section{II-3. Crack Propagation Studies}

Perhaps the greatest, catastrophic failure mode that could befall DOE Liquid Waste storage tanks is stress corrosion cracking; specifically, caustic cracking (CC). This process usually occurs in high strength carbon or low alloy steels, in the heat affected zones adjacent to welds in these materials. In any event, crack propagation rates can be very high, thus leading to wall penetration soon after initiation. Despite the obvious importance of $\mathrm{CC}$ in any industry that handles concentrated hydroxide solutions (e.g., extraction of alumina from bauxite $[1,2]$ ) little is known of the mechanistic details of this crack propagation process.

Failure by caustic cracking of the carbon steel storage tank must be considered as one of the principal threats to tank integrity. An enormous literature exists on the 
environment assisted cracking (EAC) of carbon steels in caustic solutions, but the work is almost entirely phenomenological in nature. For example, it is not always clear whether crack propagation occurs via anodic dissolution or HIC, or a combination of both, but the bulk of the evidence suggests that HIC is the fracture mechanism. Thus, it has long been observed that cracks propagate via discrete events and this is often taken as being indicative of the role of hydrogen [4]. Thus, hydrogen is postulated to produce a brittle matrix at the crack tip that undergoes periodic fracture to produce intermittent crack advance [4]. HIC occurs with a fracture dimension that is determined by the time between events and the diffusion length of hydrogen in the matrix (typically a few microns). SCC may also occur by cyclical slip/dissolution/ repassivation $[5,6]$ and hence exhibits periodic advance, but the slip dimension should be of the order of some low multiple of the Burger's vector (a few nanometers) and is unlikely to give rise to the abrupt transient in current that has been found in this study. Thus, from a mechanistic viewpoint, knowledge of the dimension of the fracture event is most important, for it may provide a means of differentiating between anodic dissolution and hydrogen-induced fracture. However, quantitative knowledge of the mechanism(s) of caustic cracking of carbon steel in highly concentrated alkaline solutions is quite poor. Therefore, emphasis in this part of our work is on the fundamental aspect of caustic cracking mechanisms by using electrochemical emission spectroscopy (EES) on the coupling current that flows between the crack and the external surfaces to ascertain the dimension of the brittle micro-fracture events that occur during crack propagation. The well established acoustic emission technique (AET) and the direct current potential drop technique (DCPD) have also been used to simultaneously monitor the crack propagation.

Acoustic emission (AE) is the transient elastic radiation produced by many microscopic deformation and fracture processes in materials. When this elastic radiation contacts a surface, it produces a displacement that can be detected and measured by a suitable sensor. AE technology involves the use of ultrasonic transducers $(20 \mathrm{KHz}-1$ $\mathrm{MHz}$ ) to listen for the sounds of failure occurring in materials and structures. Acoustic emission sensors, which are usually made from piezoceramics, convert displacement to voltage which is amplified and subsequently stored or analyzed. Typical AE signals 
appear as an oscillating voltage that quickly rises to a peak value, and then slowly decays to the background noise level.

Acoustic emission has been detected in many materials during elastic and plastic deformation and fracture. Possible sources of these elastic waves include the rapid, collective motion of a large number of dislocations, inclusion and precipitate fracture or pull-out of the matrix, lüder band propagation in iron alloys, and rapid, brittle crack extension. Because many of these processes occur during sub-critical crack growth in materials, acoustic emission has a potential to be a monitor of crack initiation and propagation in engineering components [7-14]. The acoustic emission technique (AET) is a very useful tool for monitoring stress corrosion cracking, which is evident from the vast literature that is available [7, 9-14]. In the current research, AET is being employed to monitor the stress corrosion crack growth. Apart from crack growth studies, AET is an effective tool to detect crack initiation and crack depth, the details of which are given in the results section. Results from these experiments can be effectively used to corroborate the results obtained from EES.

Direct current potential drop (DCPD) technique is one of the most common and sensitive in situ crack monitoring techniques [15]. The potential drop (PD) method for crack measurement involves the measurement of potential differences on the surface of a body which is conducting an electric current. The magnitude of the potential difference is dependent upon many factors, but assuming the current source is stable, the crosssectional area of the conducting path between the two measurement points largely determines the magnitude of the potential drop between them. If a crack forms between the measurement points, the conducting path lengthens (i.e. the cross-sectional area decreases) and the potential drop increases. It is possible to calibrate this change in potential drop with the increase in crack depth. By making a series of PD measurements along the crack, the depth profile or shape of the crack front can be determined and plotted and, over the course of a test, crack growth rates can be calculated.

In order to carry out the study in a parametric manner, the simple $\mathrm{NaOH} / \mathrm{H}_{2} \mathrm{O}$ electrolyte system was used. A high-strength, low-alloy steel (AISI 4340 [UNS G43400 (1)]) was chosen for the test material and was heat-treated to represent the HAZ adjacent to welds in DOE storage tanks. Methods have been developed to measure the 
electrochemical emissions in the coupling current from growing cracks in a high-strength, low alloy steel. These techniques are expected to provide fundamental information on the mechanisms of crack propagation. The method used is to measure the coupling current that flows between the crack and the external surface, as a crack propagates through the steel (Figure II-3.1).

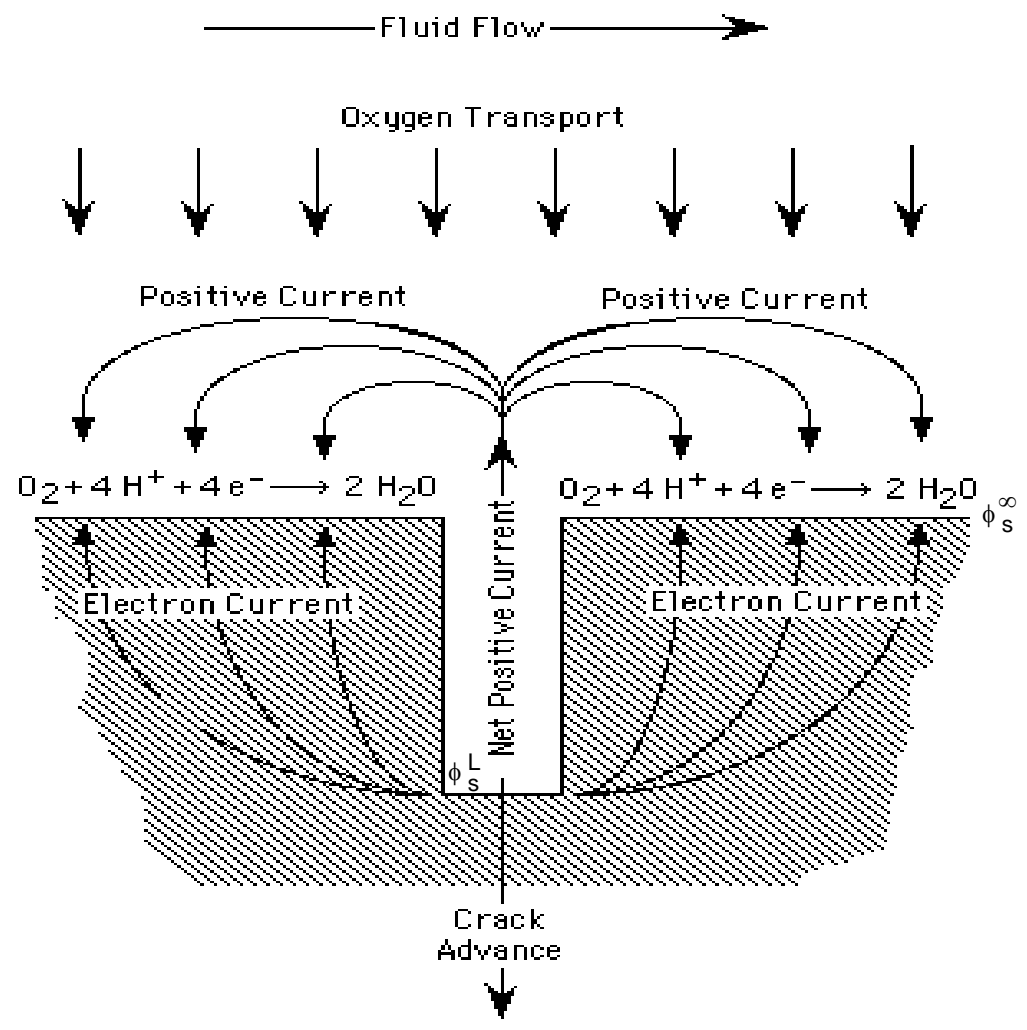

Figure II-3.1. Schematic of the origin of the coupling current in stress corrosion cracking. The coupling current is required by the differential aeration hypothesis for localized corrosion and the conservation of charge requires that the electron current flowing through the substrate from the crack to the external surface must be equal to the positive ionic current flowing through the solution from the crack to the external surface.

A coupling current of appropriate magnitude is required by the differential aeration hypothesis to satisfy charge conservation in the system [16]. Previous work on SCC in Type 304 stainless steel ([UNS 30400] SS) in high-temperature aqueous systems shows that the noise in the coupling current can be attributed to brittle micro-fracture events that have a dimension on the order of $3 \mu \mathrm{m}$ and that these fracture events occur in "packages" of 4 to 13 , corresponding to the grain size of the steel [6]. The present work seeks to 
determine whether similar events can be detected in the coupling current that is produced in the fracture of high strength, low-alloy steels in simulated DOE waste environments. Simultaneous monitoring of the acoustic emission signals would help to verify if the individual transients in the coupling current actually correspond to a microfracture event. Detection and characterization of the noise in the coupling current is expected to provide mechanistic information of unprecedented value.

\section{II-3 References:}

1. H.H. Le, E. Ghali, Corrosion Science, 35, n 1, 435 (1993).

2. R.K. Singh Raman, B.C. Muddle, Materials Science and Technology, 19, n 5, $642(2001)$

3. G. L. Edgemon, M. J. Danielson, and G. E. C. Bell, J. Nuclear Materials, 245, 201 (1997).

4. M. P. Manahan, Sr, D. D. Macdonald and A. J. Peterson, Jr., Corros. Sci, 37, 189 (1995).

5. D. A. Vermilyea and R. B. Diegle, Corrosion, 32, 26 (1976).

6. J. C. Scully, Corros. Sci., 20, 997 (1980).

7. D.J. Mills, and S. Mabbutt, Prog. Org. Coat, 39, 41 (2000)

8. R.H. Jones, M.A. Friesel, and W.W. Gerberich, Met trans A, 20A, 637, (1989)

9. A. Berkovits and D. Fang, Engg. Fra. Mech, 51, 401 (1995)

10. F. Ferrer, E. Schille, D. Verardo and J. Goudiakas, JOM, 37, 2707 (2002)

11. K.Y. Sung, I.S. Kim and Y.K. Yoon, Scripta Materialia, 37, 1255 (1997)

12. B. Knowlton, A.M Siddiqui and S. Jihan, British Corrosion Journal, 32, 249 (1997)

13. R.H. Jones, M.A. Friesel and R. Pathania, Corrosion, 47, 105 (1991)

14. N.K. Mukhopadyay, G. Sridhar, N. Parida, S. Tarafder and V.R. Ranganath, Engineering Failure Analysis, 6, 253 (1999)

15. ASM Handbook on corrosion

16. D. D. Macdonald and M. Urquidi-Macdonald, Corros. Sci., 32, 51 (1991) 


\section{II-4 Electrochemical Emission Spectroscopy}

\section{II-4.1 Generation of EC Noise from General and Localized Corrosion}

Currently an electrochemical cell is being constructed for the collection of EC noise from general and localized corrosion. The cell's purpose is to model the environment inside the waste storage containers. The cell simulates the electrochemical environment and the temperature of the containers such that electrochemical noise data relating to general and localized corrosion of the type from the actual containers is generated. This electrochemical noise is collected for later analysis along with other types of corrosion noise data.

\section{II-4.2 Computer Simulation of Anodic and Cathodic Activity}

The EC noise collected from the electrochemical cell is also being used to further the development of the model describing corrosion in the storage containers. It is the basis for computer modeling of the anodic and cathodic activity on the surface of the steel specimens. In the simulation, the surface of the sample is represented by a grid of possible sites. Initially a completely random arrangement of anodic and cathodic sites is produced, figure II-3.1.

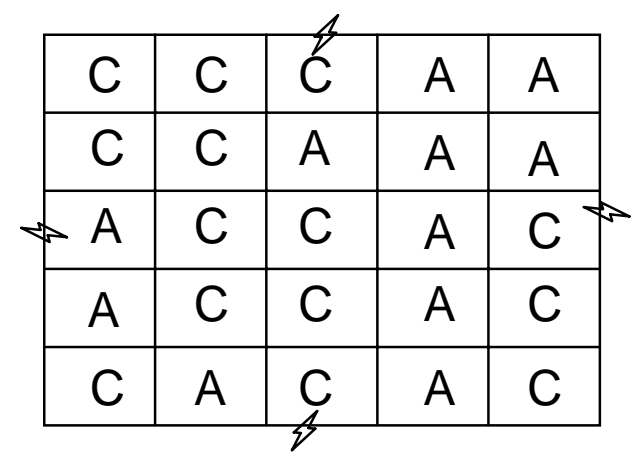

Figure II-3.1. Random anodic and cathodic sites 
Whether an anodic site remains anodic or switches to a cathodic state from moment to moment is also initially completely random. The total number of anodic and cathodic sites is regulated by conservation of charge, thus it is always necessary to maintain charge equality across the entire simulated specimen surface. The EC processes are modeled mathematically by the following equations:

Anodic process: $\mathrm{I}_{\mathrm{a}}=\mathrm{N}_{\mathrm{a}} \mathrm{i}_{\mathrm{o}, \mathrm{a}} \mathrm{e}^{\wedge}\left(\mathrm{E} / \mathrm{b}_{\mathrm{a}}\right)$

Cathodic process: $I_{c}=N_{c} i_{o, c} e^{\wedge}\left(E / b_{c}\right)$

Where $\mathrm{N}_{\mathrm{a}}, \mathrm{N}_{\mathrm{c}} \quad$ : initial number of anodic and cathodic sites, respectively $\mathrm{i}_{\mathrm{o}, \mathrm{a}}$, io,c : initial anodic and cathodic current, respectively E : potential $b_{a}$ and $b_{c}$ : anodic and cathodic Tafel slopes, respectively

$\Delta \mathrm{I}$ can be derived from these equations to be

$$
\Delta \mathrm{I}=\mathrm{i}_{\mathrm{o}, \mathrm{a}} \mathrm{e}^{\wedge}(\mathrm{E}+\Delta \mathrm{E}) / \mathrm{b}_{\mathrm{a}}-\mathrm{i}_{\mathrm{o}, \mathrm{c}} \mathrm{e}^{\wedge}-(\mathrm{E}+\Delta \mathrm{E}) / \mathrm{b}_{\mathrm{c}}
$$

But when $\Delta \mathrm{E} / \mathrm{b}_{\mathrm{a}}, \Delta \mathrm{E} / \mathrm{b}_{\mathrm{c}}<<1$

$$
\Delta \mathrm{I}=\mathrm{i}_{\mathrm{o}, \mathrm{a}} \mathrm{e}^{\wedge}\left(\mathrm{E}_{\text {corr }} / \mathrm{b}_{\mathrm{a}}\right)\left(1+\Delta \mathrm{E} / \mathrm{b}_{\mathrm{a}}\right)-\mathrm{i}_{\mathrm{o}, \mathrm{c}} \mathrm{e}^{\wedge}-\left(\mathrm{E}_{\mathrm{corr}} / \mathrm{b}_{\mathrm{c}}\right)\left(1-\Delta \mathrm{E} / \mathrm{b}_{\mathrm{c}}\right)
$$

Where $\mathrm{E}_{\text {corr }} \quad$ : potential at combined anodic and cathodic current

The current and potential from this random arrangement of sites is transformed using a mathematical method like the Fast Fourier Transform, the Maximum Entropy Method, or Wavelet Analysis (which will be discussed later) into a power spectrum density like that of any non-simulated data set. The next step in the computer simulation is to change the associations between the anodic and cathodic sites. Instead of the random arrangement used initially, the sites can be arranged more regularly, figure II-4.2, or cathodic sites can be given an improved probability of occurring adjacent to an anodic site, figure II-4.3. 


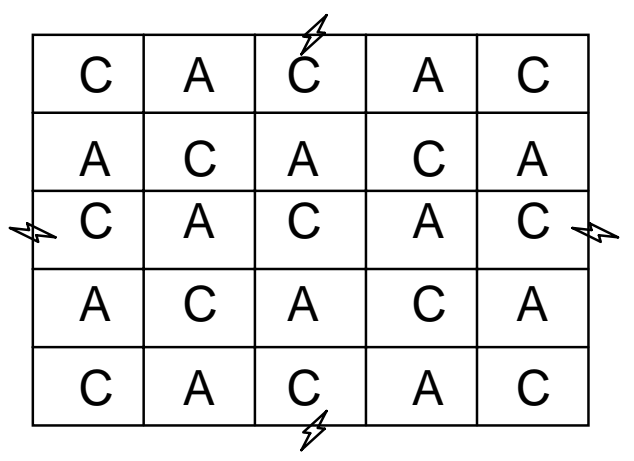

Figure II-4.2. Regular initial positions

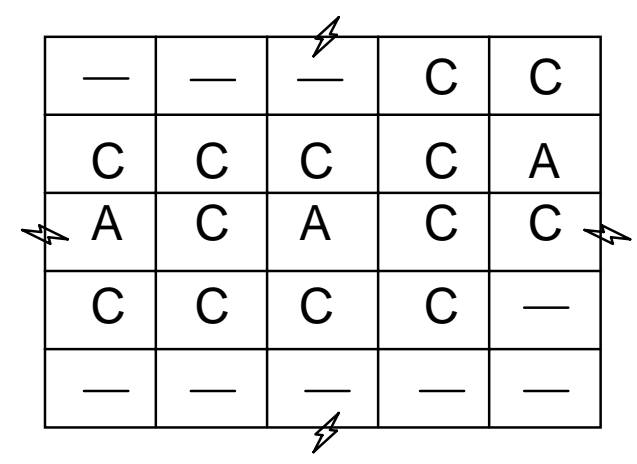

Figure II-4.3. Ordered initial positions

These both more accurately represent the possible arrangement of sites according to the PDM model [1]. In addition to changing the arrangement of sites and their probability of proximity, the likelihood of sites switching character (from anodic to cathodic, or visa versa) from moment to moment is manipulated to better represent the behavior of anodic sites transitioning from metastable to stable or inactive sites, also according to the PDM model. Figure II-4.4 illustrates a site in column 3 transitioning from anodic to a cathodic or inactive site.

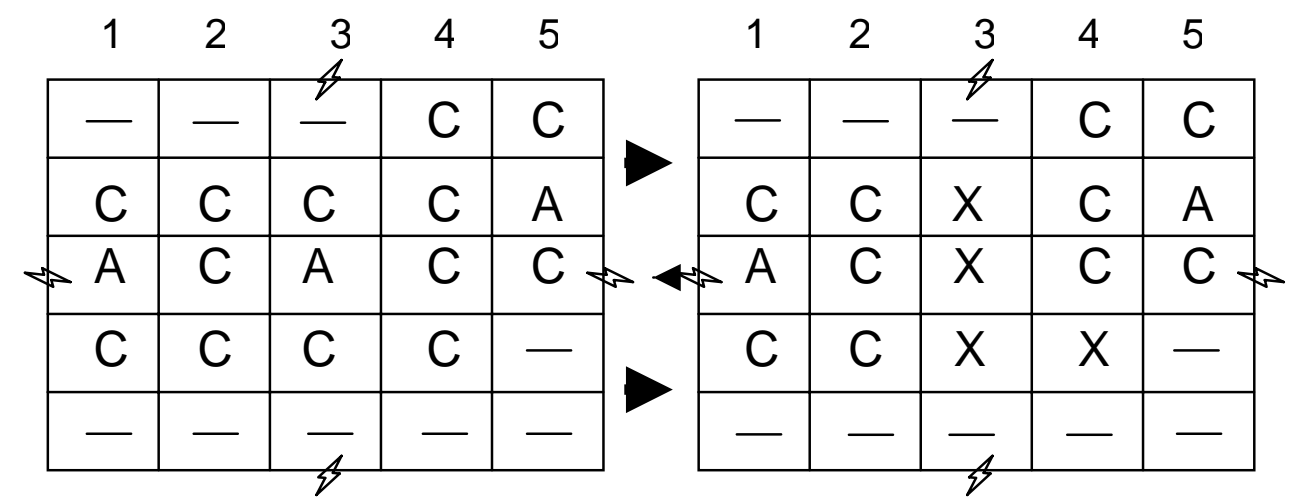

Figure II-4.4. Sites transitioning

By manipulating the rules governing the simulation, it will ultimately produce a data set and PSD plot resembling that of the natural localized corrosion processes. At this point, the rules of the simulation will provide a greater understanding of the natural process of localized corrosion, and will help refine the mathematical models of and predicting corrosion.

\section{II-4.3 Wavelet Analysis}


In the analysis of electrochemical noise data, there are a variety of methods available, some of which are mentioned above. The Fast Fourier Transformation (FFT), for example, converts data from the normal (temporal) domain into the Fourier (frequency) domain. This does two things: one, it can make it easier to compute the solution for a function that is difficult to solve in the time domain, and two, in the case of signal analysis it allows for the identification of the functions that comprise the initial signal. Fourier transformations use a combination of sine and cosine waves, superimposed on one another, to rebuild the initial signal.

The problem with FFT analysis is that it assumes a steady state. A data set which contains transients, or one in which the mean value of the data varies over time cannot be properly analyzed in FFT [2]. The signal must be normalized by subtracting out (or adding to) the changing mean to bring it to a steady state, and there is no information provided in the final analysis about the time (the point in the temporal domain) at which any of the comprising functions were contributing. Wavelet Analysis performs in a similar fashion to FFT, determining a set of "wavelets" or "basis functions" that comprise the original signal, but Wavelet Analysis is superior in that the basis function chosen for the analysis is limited in the time domain. This allows for the entire data set to be analyzed without losing information about an evolving signal (like electrochemical noise signals), and information is provided about the point in the time domain that a specific function was active.

Wavelet analysis works by dividing the data into two sets: one set is the "smooth" information (the baseline function), and the other set is the "detail" information. These two sets are given by wavelet coefficients; 2 for the smooth information and an integral power of 2 for the detail information (depending on how much detail is necessary) [3]. These coefficients are key to Wavelet Analysis's superiority over previous analysis methods in terms of monitoring and predicting corrosion.

Current research into corrosion and the electrochemical noise associated with each type focuses on the plateau height, roll-off slope, and roll-off frequency of the PSD plots. Research suggests that a roll-off slope of less than $20 \mathrm{~dB} /$ decade is indicative of pitting corrosion while a greater slope results from general corrosion or SCC [4]. Wavelet analysis can provide the same PSD plot that FFT or MEM can provide but it has one 
additional advantage. Comparing two PSD data plots like B and C, figure II-4.5, FFT and MEM cannot distinguish between the two because their features are very similar (slope and plateau height), but using Wavelet Analysis, the coefficients provided in figure II-4.6 show a clear difference between the two data sets [5].

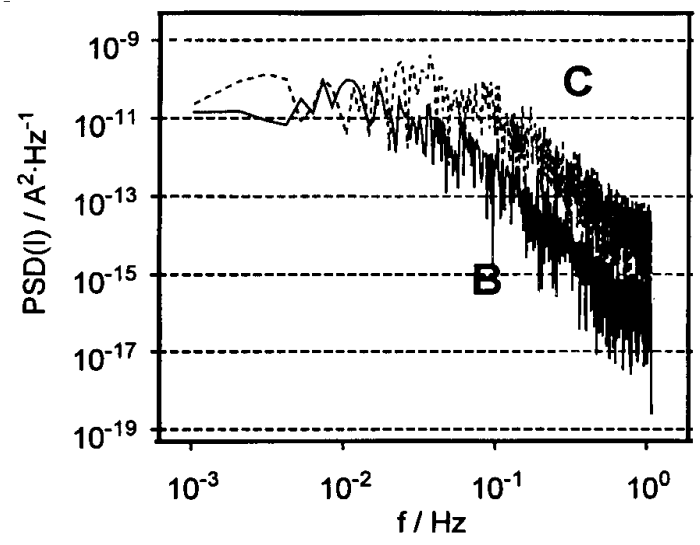

Figure II-4.5. Similar PSD plots [5]

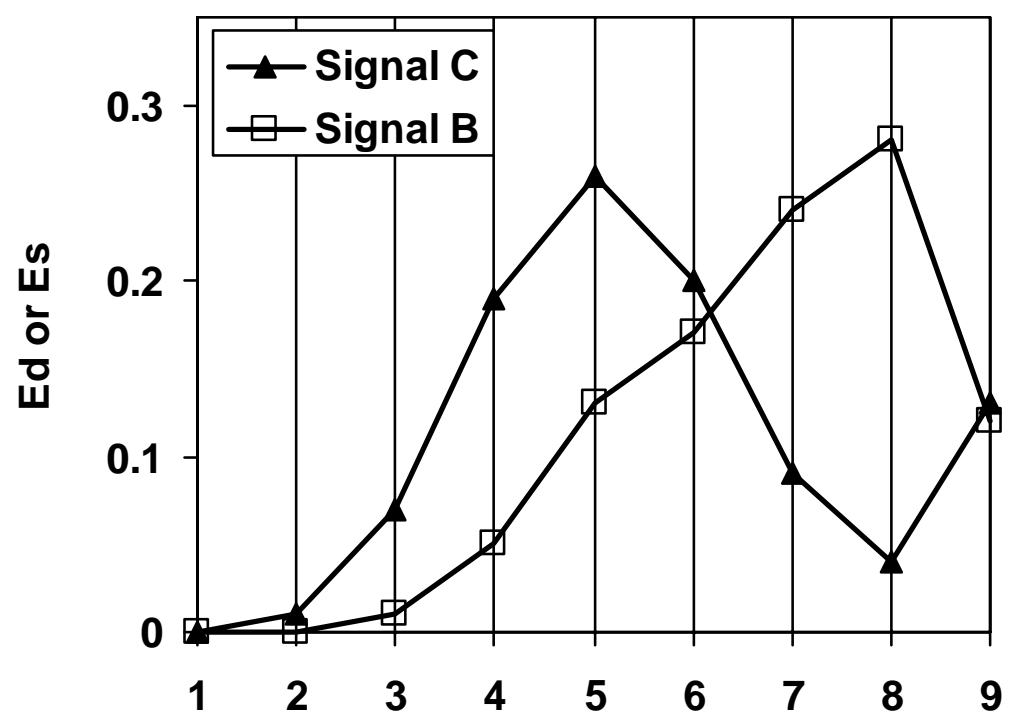

Figure II-4.6. Wavelet coefficients [5]

This quality, along with the others mentioned above, is why Wavelet Analysis will be used in the third part of this localized corrosion research. Wavelet Analysis is being performed on the general corrosion and SCC noise data from the current research, and will soon be used on the localized corrosion data as well. By comparing the PSD 
plots, their qualities, and the wavelet coefficients, it will be possible for the first time to definitively differentiate between the types of corrosion occurring in real-time.

\section{II-4 References}

1. D. D. Macdonald, Pure Appl. Chem., 71, 951 (1999).

2. R.A. Cottis, Corrosion, 57, 3 (2001).

3. W.H. Press, et al. Numerial Recipies in C++. Cambridge University Press, 2002. p. 599

4. Jun Liu and D.D. Macdonald, "Electrochemical Emission Spectroscopy of AISI 1018 Carbon Steel." Pennsylvania State University, 2002.

5. A. Aballe et al., Electrochimica Acta, 44, 4805 (1999).

\section{II-5 Unification of the Deterministic and Statistical Approaches for Predicting Localized Corrosion Damage (Damage Function Analysis-DFA)}

In this section of the project, we provide an alternative, more general theoretical basis for Damage Function analysis (DFA), by drawing an analogy between the growth of a pit and the movement of a particle. In contrast to our previous formulation of DFA, which was developed specifically for enabling the damage function for localized corrosion to be calculated from the Point Defect Model for passivity breakdown, the Coupled Environment Pitting Model for pit growth, and the theory of prompt and delayed repassivation, the new formulation readily incorporates any theories or models (deterministic or empirical) for these stages in the development of a pit. We show that the new formulation leads to the original expressions for the damage functions for active (living) and passivated (dead) pits, and hence for the differential and integral damage functions, as were obtained from the original theory. We also describe the unification of deterministic (Damage Function Analysis, DFA) and empirical, statistical (Extreme Value Statistics, EVS) methods for predicting the development of localized corrosion damage on metal surfaces. In particular, we have devised a means of estimating the central and scale parameters of EVS directly from DFA in a "first principles" manner, as well as from fitting the EVS distribution function to experimental data for short times, in order to predict the extreme value distributions at longer times. The techniques have 
been evaluated on EVS data for the pitting of manganese steel in $\mathrm{CO}_{2}$-acidified seawater and for the pitting of aluminum in tap water. Finally, we outline the generalization of pit nucleation, as described by the Point Defect Model, for external conditions that depend on time.

It will be shown from DFA that, with certain simplifying assumptions, the distribution of the deepest pit among "identical" specimens must be described by extreme value statistics (EVS). A combination of DFA and traditional statistical analysis (SA) offers significant advantages over purely statistical/empirical approaches that are not based upon deterministic principles. Thus, DFA allows us to express the fitting parameters for SA in terms of values for the physical parameters of the component deterministic model(s) (e.g. pit nucleation and propagation rates, repassivation constants, etc.) that can be validated by independent experiment. Accordingly, it becomes possible to predict the statistical fitting parameters as the external conditions change with time.

\section{Research Progress}

\section{III-1 Task: Continued Development of the Theory of Passivity}

The objective of this task is to continue the development of the theory of passivity based on the Point Defect Model (PDM) [1], in order to provide a basis for interpreting experimental data on the general and localized corrosion of metals and alloys in DOE liquid waste environments. Previous work on this project was able to account for the transients in barrier layer thickness and current upon perturbing the $\mathrm{pH}$ and the potential. The current work is concentrating on the effect that temperature has on the transients. In addition we are continuing to take data for various $\mathrm{pH}$ 's and potentials to ensure the accuracy of any predictions. The theory is also being extended to account for the electrochemical impedance characteristics of iron (and other passive metals) in which the dissolution of the barrier layer occurs. These models are being used to interpret impedance data for iron and other metals in borate buffer solutions under conditions where the outer layer forms or where it is prevented from forming (e.g. addition of EDTA). Optimization procedures are being used to fit the impedance models to the experimental impedance data, which are measured as functions of potential, $\mathrm{pH}$, and 
temperature in order to derive various model parameters as functions of the same independent variables. Our ultimate goal is to derive a single set of parameters that will account for the growth and breakdown of the passive film on iron over the entire range of conditions that are of interest in the storage of DOE liquid waste.

Eventually, the kinetic parameters will be incorporated into Damage Function Analysis which can predict passivity breakdown. Earlier work on some metals suggests that pit formation is due to the coalescence of cation vacancies at the metal/film interface. This previous work shows that once the critical vacancy density is reached, the film ruptures to form a pit. Based upon the kinetic parameters derived for the general corrosion case, two constants relating to the cation vacancy coalescence can be calculated. These two constants can then be applied to predict passivity breakdown. Therefore, experiments are being performed to measure pitting as a function of potential, chloride ion concentration, and temperature in high $\mathrm{pH}$ solutions, similar to that in the DOE liquid waste containers.

The immediate tasks are as follows:

1. Using EIS and ellipsometry to extract the kinetic parameters

a. Deriving an equation for the impedance of the passive film formed on iron based on the PDM

b. Obtaining impedance and thickness data for iron

c. Measuring the relative amounts of the various iron cation species present in the film via X-ray Photon Spectroscopy (XPS)

2. Using DataFit (Curve fitting software) to optimize the impedance data to the derived equation for obtaining the kinetic information

3. Obtaining data to be incorporated in DFA

\section{III-1.1a,b. Deriving the Impedance Equations from PDM}

\section{Task Status}

The following part of this work is dedicated to the development of an impedance model based on the Point Defect Model (PDM) [4] in order to perform mechanistic 
analysis of impedance data for passive iron. Professor Macdonald provided the model and basic equations to the author.

Figure III-1.1 summarizes the physicochemical processes occurring within the passive barrier film formed on iron, in terms of the PDM. Reaction (1) describes the injection of $\mathrm{Fe}_{i}^{\chi^{+}}$into the barrier layer as an interstitial, and then the iron interstitial is transmitted through the barrier layer and ejected into the solution as presented by Reaction (3). Reaction (2) results in the growth of the barrier layer into the bulk metal iron, and Reaction (5) leads to the destruction of the barrier layer by dissolution. Reaction (4) is the consumption of oxygen vacancy at the film/solution interface, which is produced by Reaction (2) at the metal/film interface. Note that Reactions (1), (3), and (4) are lattice conservative processes, while Reactions (2) and (5) are lattice nonconservative processes. A steady state involves two non-conservative reactions, since only one non-conservative reaction would lead to monotonic growth or thinning of the passive film. Considering our previous findings that the dominant defects in the barrier layer of passive iron must be oxygen vacancies, or iron interstitials, or both, due to the $n$ type electronic character of the passive film, the reactions regarding the consumption (at the metal/film interface) and production (at the film/solution interface) of iron vacancies are not included in the impedance model development






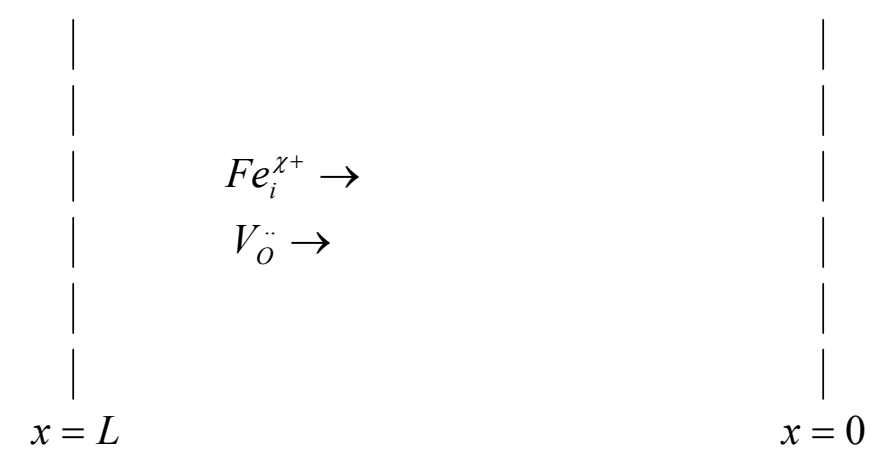

Figure III-1.1. Schematic of physicochemical processes that occur within a barrier oxide $\left(\mathrm{Fe}_{3} \mathrm{O}_{4}\right)$ layer on passive iron according to the Point Defect Model. $\mathrm{Fe} \equiv$ iron atom; $\mathrm{Fe}_{i}^{\chi+} \equiv$ interstitial iron cation; $V_{F e} \equiv$ iron vacancy at the metal phase; $F e_{F e} \equiv$ iron cation in a normal cation position; $\mathrm{Fe}^{\Gamma+} \equiv$ iron cation at the solution phase; $V_{O} \equiv$ oxygen vacancy; $O_{O} \equiv$ oxygen ion in anion site.

The film thickness change with time involves two lattice non-conservative reactions, consequently determined as follows:

$$
\frac{d L}{d t}=-\frac{2 \Omega}{\chi} J_{o}-\Omega k_{5} C_{H^{+}}^{n}
$$

in which $\Omega=14.9 \mathrm{~cm}^{3} \mathrm{~mol}^{-1}$ is the volume per mole of the barrier layer, $\chi=8 / 3$ is the oxidation state of iron in the barrier layer, $J_{o}$ is the flux for oxygen vacancies within the barrier layer, $C_{H^{+}}$is the hydrogen ion concentration in the solution at the film/solution interface, $n$ is the kinetic order of the film dissolution reaction with respect to $C_{H^{+}}$at the film/solution interface, and $k_{5}$ is the rate constant for Reaction (5). Table III-1.1 displays rate constants for five interfacial reactions; the definition of standard rate constants will be detailed later in this section.

All electron-related interfacial reactions contribute to the total current density, which is described as 
$I=F\left[-\chi J_{i}^{m / f}-2 J_{o}^{m / f}-(\Gamma-\chi) J_{i}^{f / s}+(\Gamma-\chi) k_{5} C_{H^{+}}^{n}\right]$

in which $F=96487 \mathrm{C} / \mathrm{mol}$ is Faraday's constant, and $\Gamma=3$ is the oxidation state of iron in solution. The fluxes are written as $J_{i}=-D_{i} \frac{\partial C_{i}}{\partial x}-\chi K D_{i} C_{i}$ for iron interstitials and $J_{o}=-D_{o} \frac{\partial C_{o}}{\partial x}-2 K D_{o} C_{o}$ for oxygen vacancies, where $D$ and $C$ are diffusivity and concentration, subscripts $i$ and $o$ are for iron interstitials and oxygen vacancies, $K=\varepsilon F / R T, R=8.314 \mathrm{Jmol}^{-1} \mathrm{~K}^{-1}$ is the gas constant, $T$ is the absolute temperature, and $\varepsilon$ is the electric field strength, which is considered a constant according to the PDM. The continuity equations $\frac{\partial C}{\partial t}=-\nabla J$ then become

$$
\begin{aligned}
& \frac{\partial C_{i}}{\partial t}=D_{i} \frac{\partial^{2} C_{i}}{\partial x^{2}}+\chi D_{i} K \frac{\partial C_{i}}{\partial x} \\
& \frac{\partial C_{o}}{\partial t}=D_{i} \frac{\partial^{2} C_{o}}{\partial x^{2}}+2 D_{o} K \frac{\partial C_{o}}{\partial x}
\end{aligned}
$$

the initial conditions $(\mathrm{t}=0)$ of which are $C_{i}(x)=C_{i}^{0}(x)$ and $C_{o}(x)=C_{o}^{0}(x)$ and the boundary conditions of which are: at $x=0$ (the film/solution interface): $J_{i}(0)=-k_{3}^{0} e^{a_{3} V} C_{i}(0)\left(k_{3}^{0}\right.$ is in $\left.\mathrm{cm} / \mathrm{s}\right) ; J_{o}(0)=-\left(\frac{\chi}{2}\right) k_{4}^{0} e^{a_{4} V} C_{o}(0)\left(k_{4}^{0}\right.$ is in $\left.\mathrm{cm} / \mathrm{s}\right)$; at $x=L \quad$ (the metal/film interface): $J_{i}(L)=-k_{1}^{0} e^{a_{1} V} e^{-b_{1} L} \quad\left(k_{1}^{0} \quad\right.$ is in $\left.\mathrm{mol} / \mathrm{s} / \mathrm{cm}^{2}\right)$; $J_{o}(L)=-\left(\frac{\chi}{2}\right) k_{2}^{0} e^{a_{2} V} e^{-b_{2} L}\left(k_{2}^{0}\right.$ is in $\left.\mathrm{mol} / \mathrm{s} / \mathrm{cm}^{2}\right)$.

Combined with the rate constants shown in Table III-1.1, the rate of film thickness change in Equation (1) and current density in Equation (2) then become 


$$
\begin{gathered}
\frac{d L}{d t}=-\Omega k_{2}^{0} e^{a_{2} V} e^{-b_{2} L}-\Omega k_{5}^{0} e^{a_{5} V} C_{H^{+}}^{n} \\
I=F\left[\chi k_{1}^{0} e^{a_{1} V} e^{-b_{1} L}+\chi k_{2}^{0} e^{a_{2} V} e^{-b_{2} L}+(\Gamma-\chi) k_{3}^{0} e^{a_{3} V} C_{i}(0)+(\Gamma-\chi) k_{5}^{0} e^{a_{5} V} C_{H^{+}}^{n}\right]
\end{gathered}
$$

Note that the current density is a function of $V, L, C_{i}(0)$. Accordingly, for any arbitrary changes $\delta V, \delta L, \delta C_{i}(0)$

$\delta I=\left(\frac{\partial I}{\partial V}\right)_{L, C_{i}(0)} \delta V+\left(\frac{\partial I}{\partial L}\right)_{V, C_{i}(0)} \delta L+\left(\frac{\partial I}{\partial C_{i}(0)}\right)_{V, L} \delta C_{i}(0)$

in which the variations are sinusoidal in terms of EIS, i.e., $\delta V=\Delta V e^{j \omega t} ; \delta L=\Delta L e^{j \omega t}$; $\delta C_{i}(0)=\Delta C_{i}(0) e^{j \omega t}$, note that $\Delta x$ is the amplitude of the variation in $X$ at a frequency $\omega=0$.

Table III-1.1. Rate constants $k_{i}=k_{i}^{0} e^{a_{i} V} e^{-b_{i} L}$ for five interfacial reactions in terms of the Point Defect

\begin{tabular}{|c|c|c|c|}
\hline Reaction & $\begin{array}{l}a_{i} \\
\left(V^{-1}\right)\end{array}$ & $\begin{array}{l}b_{i} \\
\left(\mathrm{~cm}^{-1}\right)\end{array}$ & Unit of $k_{i}^{0}$ \\
\hline $\begin{array}{l}\text { (1) } \\
\mathrm{Fe} \stackrel{k_{1}}{\longrightarrow} \mathrm{Fe}_{i}^{\chi+}+V_{\mathrm{Fe}}+\chi e^{\prime}\end{array}$ & $\alpha_{1}(1-\alpha) \chi \gamma$ & $\alpha_{1} \chi K$ & $\frac{\mathrm{mol}}{\mathrm{cm}^{2} \mathrm{~s}}$ \\
\hline $\begin{array}{l}\text { (2) } \\
\mathrm{Fe} \stackrel{k_{2}}{\longrightarrow} \mathrm{Fe}_{\mathrm{Fe}}+\frac{\chi}{2} V_{\ddot{o}}+\chi e^{\prime}\end{array}$ & $\alpha_{2}(1-\alpha) \chi \gamma$ & $\alpha_{2} \chi^{K}$ & $\frac{\mathrm{mol}}{\mathrm{cm}^{2} \mathrm{~s}}$ \\
\hline $\begin{array}{l}\text { (3) } \\
\mathrm{Fe}_{i}^{\chi+} \stackrel{k_{3}}{\longrightarrow} \mathrm{Fe}^{\Gamma+}+(\Gamma-\chi) e^{\prime}\end{array}$ & $\alpha_{3} \alpha \Gamma \gamma$ & 0 & $\frac{\mathrm{cm}}{\mathrm{s}}$ \\
\hline
\end{tabular}
Model. 


\begin{tabular}{|l|l|l|l|}
\hline$(4)$ & $2 \alpha_{4} \alpha \gamma$ & 0 & $\frac{\mathrm{cm}}{\mathrm{s}}$ \\
$\mathrm{V}_{O}+\mathrm{H}_{2} \mathrm{O} \stackrel{k_{4}}{\longrightarrow} \mathrm{O}_{O}+2 \mathrm{H}^{+}$ & $\alpha_{5} \alpha(\Gamma-\chi) \gamma$ & 0 & $\frac{m o l^{0.4}}{\mathrm{~cm}^{0.2} s}$ \\
\hline$(5)$ & $\mathrm{FeO}_{\chi / 2}+\chi \mathrm{H}^{+} \stackrel{k_{5}}{\longrightarrow} \mathrm{Fe}^{\Gamma+}+\frac{\chi}{2} \mathrm{H}_{2} \mathrm{O}+(\Gamma-\chi) e^{\prime}$ & & \\
\hline
\end{tabular}

From Equation (7), the Faradic admittance is defined as

$$
Y_{f}=\frac{\delta I}{\delta V}=I^{V}+I^{L} \frac{\Delta L}{\Delta V}+I^{C_{i}(0)} \frac{\Delta C_{i}(0)}{\Delta V}
$$

More simply stated, $I^{V}=\left(\frac{\partial I}{\partial V}\right)_{L, C_{i}(0)} ; I^{L}=\left(\frac{\partial I}{\partial L}\right)_{V, C_{i}(0)} ; I^{C_{i}(0)}=\left(\frac{\partial I}{\partial C_{i}(0)}\right)_{L, V} . \quad$ These differentials will be evaluated later from Equation (6).

Now we return to Equation (5) and determine the response of $\frac{d L}{d t}$ to $\delta V, \delta L$, and $\delta C_{i}(0)$ by taking the total differential. Thus, for the relaxation in film thickness: $\frac{d}{d t}(\delta L)=\Omega k_{2}^{0} a_{2} e^{a_{2} V} e^{-b_{2} L} \delta V-\Omega k_{2}^{0} b_{2} e^{a_{2} V} e^{-b_{2} L} \delta L-\Omega k_{5}^{0} a_{5} e^{a_{5} V} C_{H^{+}}^{n} \delta V$. Considering that $\frac{d}{d t}(\delta L)=j \omega \Delta L e^{j w t}$, so that $j \omega \Delta L e^{j \omega t}=\Omega\left(k_{2}^{0} a_{2} e^{a_{2} V} e^{-b_{2} L}-k_{5}^{0} a_{5} e^{a_{5} V} C_{H^{+}}^{n}\right) \Delta V e^{j \omega t}-\Omega k_{2}^{0} b_{2} e^{a_{2} V} e^{-b_{2} L} \Delta L e^{j \omega t}$, and finally

$$
\frac{\Delta L}{\Delta V}=\frac{\Omega\left(k_{2}^{0} a_{2} e^{a_{2} V} e^{-b_{2} L}-k_{5}^{0} a_{5} e^{a_{5} V} C_{H^{+}}^{n}\right)}{\Omega k_{2}^{0} b_{2} e^{a_{2} V} e^{-b_{2} L}+j \omega} \text { or } \frac{\Delta L}{\Delta V}=\frac{\Phi_{2}}{1+j \omega \tau_{2}}
$$




$$
\begin{aligned}
& \text { where } \quad \Phi_{2}=\left(\frac{a_{2}}{b_{2}}\right)-\left(\frac{k_{5}^{0}}{k_{2}^{0}}\right)\left(\frac{a_{5}}{b_{2}}\right) e^{\left(a_{5}-a_{2}\right) V} e^{b_{2} L} C_{H^{+}}^{n} \\
& \qquad \tau_{2}=\frac{1}{\Omega k_{2}^{0} b_{2} e^{a_{2} V} e^{-b_{2} L}}
\end{aligned}
$$

For iron interstitials, the relaxation for $C_{i}(0)$ is determined through the mass balance at the film/solution interface $\frac{d C_{i}(0)}{d t}=-k_{3}^{\prime} C_{i}(0)=-k_{3}^{0^{0}} e^{a_{3} V} C_{i}(0)\left(\right.$ Unit of $k_{3}^{\prime 0}$ is $\left.\mathrm{s}^{-1}\right)$. The total differential is $\frac{d}{d t}\left(\delta C_{i}(0)\right)=-k_{3}^{\prime 0}\left[a_{3} e^{a_{3} V} C_{i}(0) \delta V+e^{a_{3} V} \delta C_{i}(0)\right]$. Substituting in the sinusoidal variations for $\delta V$ and $\delta C_{i}(0)$,

one obtains $j \omega \Delta C_{i}(0) e^{j \omega t}=-k_{3}^{\prime 0} a_{3} e^{a_{3} V} C_{i}(0) \Delta V e^{j \omega t}-k_{3}^{\prime^{0}} e^{a_{3} V} \Delta C_{i}(0) e^{j \omega t}$ and $\Delta C_{i}(0)\left(j \omega+k_{3}^{\prime 0} e^{a_{3} V}\right)=-k_{3}^{\prime 0} a_{3} e^{a_{3} V} C_{i}(0) \Delta V$. Therefore,

$$
\frac{\Delta C_{i}(0)}{\Delta V}=\frac{-k_{3}^{\prime 0} a_{3} e^{a_{3} V} C_{i}(0)}{j \omega+k_{3}^{\prime 0} e^{a_{3} V}}=-\frac{a_{3} C_{i}(0)}{1+j \omega \tau_{3}}
$$

in which $\quad \tau_{3}=\frac{1}{k_{3}^{\prime 0} e^{a_{3} V}}$

The expression for the faradic admittance is finally derived from Equation (8) as

$$
Y_{f}=I^{V}+I^{L} \frac{\Phi_{2}}{1+j \omega \tau_{2}}-I^{C_{i}(0)} \frac{a_{3} C_{i}(0)}{1+j \omega \tau_{3}}+j \omega C
$$


in which the parallel geometric capacitance $C=\frac{\hat{\varepsilon} \varepsilon_{0}}{L}$ (dielectric constant $\hat{\varepsilon}=30$ for passive iron [12] and vacuum permittivity $\varepsilon_{0}=8.85 \times 10^{-14} \mathrm{~F} / \mathrm{cm}$ ) is taken into consideration, and

$$
\begin{aligned}
& I^{V}=\left(\frac{\partial I}{\partial V}\right)_{L, C_{i}(0)} \\
& =F\left[a_{1} \chi k_{1}^{0} e^{a_{1} V} e^{-b_{1} L}+a_{2} \chi k_{2}^{0} e^{a_{2} V} e^{-b_{2} L}+a_{3}(\Gamma-\chi) k_{3}^{0} e^{a_{3} V} C_{i}(0)+a_{5}(\Gamma-\chi) k_{5}^{0} e^{a_{5} V} C_{H^{+}}^{n}\right] \\
& I^{L}=\left(\frac{\partial I}{\partial L}\right)_{V, C_{i}(0)} \\
& =F\left[-b_{1} \chi k_{1}^{0} e^{a_{1} V} e^{-b_{1} L}-b_{2} \chi k_{2}^{0} e^{a_{2} V} e^{-b_{2} L}\right]=-\chi F\left[b_{1} k_{1}^{0} e^{a_{1} V} e^{-b_{1} L}+b_{2} k_{2}^{0} e^{a_{2} V} e^{-b_{2} L}\right] \\
& I^{C_{i}(0)}=\left(\frac{\partial I}{\partial C_{i}(0)}\right)_{V, L}=(\Gamma-\chi) F k_{3}^{0} e^{a_{3} V}
\end{aligned}
$$

In the above expressions, the parameters that appear on the right side $\left(L\right.$ and $\left.C_{i}(0)\right)$ are identified with the steady-state quantities. At steady state, Equation (3) and (4) turn into

$$
\begin{aligned}
& \frac{\partial^{2} C_{i}}{\partial x^{2}}+\chi K \frac{\partial C_{i}}{\partial x}=0 \\
& \frac{\partial^{2} C_{o}}{\partial x^{2}}+2 K \frac{\partial C_{o}}{\partial x}=0
\end{aligned}
$$

the solutions to which are

$$
\begin{aligned}
& C_{i}(x)=A_{i} e^{-\chi K x}+B_{i} \\
& C_{o}(x)=A_{o} e^{-2 K x}+B_{o}
\end{aligned}
$$


The expressions for the fluxes therefore become

$$
\begin{aligned}
& J_{i}=-\chi K D_{i} B_{i} \\
& J_{o}=-2 K D_{o} B_{o}
\end{aligned}
$$

From the boundary conditions, the coefficients are obtained as

$$
\begin{aligned}
& B_{i}=\frac{k_{1}^{0} e^{a_{1} V} e^{-b_{1} L}}{\chi K D_{i}} \\
& B_{o}=\frac{\left(\frac{\chi}{2}\right) k_{2}^{0} e^{a_{2} V} e^{-b_{2} L}}{2 K D_{o}}
\end{aligned}
$$

Because the fluxes for a given species at two interfaces are equal at steady state:

$-k_{3}^{0} e^{a_{3} V} C_{i}(0)=-k_{1}^{0} e^{a_{1} V} e^{-b_{1} L}$ and $-\left(\frac{\chi}{2}\right) k_{4}^{0} e^{a_{4} V} C_{o}(0)=-\left(\frac{\chi}{2}\right) k_{2}^{0} e^{a_{2} V} e^{-b_{2} L}$, the steady-state concentrations for iron interstitials and oxygen vacancies at the film/solution interface are

$$
\begin{aligned}
& C_{i}(0)=\left(\frac{k_{1}^{0}}{k_{3}^{0}}\right) e^{\left(a_{1}-a_{3}\right) V} e^{-b_{1} L} \\
& C_{o}(0)=\left(\frac{k_{2}^{0}}{k_{4}^{0}}\right) e^{\left(a_{2}-a_{4}\right) V} e^{-b_{2} L}
\end{aligned}
$$

In the case of steady state, Equation (1) becomes 


$$
J_{o}(L)=-\left(\frac{\chi}{2}\right) k_{5} C_{H^{+}}^{n}
$$

and from Equations (23) and (25): $-\left(\frac{\chi}{2}\right) k_{5} C_{H^{+}}^{n}=-2 K D_{o}\left(\frac{\left(\frac{\chi}{2}\right) k_{2}^{0} e^{a_{2} V} e^{-b_{2} L}}{2 K D_{0}}\right)$

Therefore, the steady-state barrier layer thickness is

$$
L=\left(\frac{a_{2}-a_{5}}{b_{2}}\right) V-\frac{1}{b_{2}} \ln \left[\left(\frac{k_{5}^{0}}{k_{2}^{0}}\right) C_{H^{+}}^{n}\right]
$$

The parameters, $k_{1}^{0}, k_{2}^{0}, k_{3}^{0}, k_{3}^{\prime 0}, k_{4}^{0}, k_{5}^{0}$, in the above equations and Table III1.1 are a function of solution $\mathrm{pH}$ and are not the standard rate constants for interfacial reactions, since the PDM defines $k_{1}^{0}=k_{1}^{00} e^{-\alpha_{1} \beta \chi \gamma p H} e^{-\alpha_{1} \chi \gamma \phi_{f / s}^{0}}, k_{2}^{0}=k_{2}^{00} e^{-\alpha_{2} \beta \chi x p H} e^{-\alpha_{2} \chi \gamma \varphi_{f}^{0} / s}$,

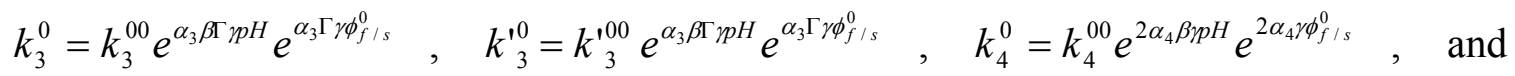
$k_{5}^{0}=k_{5}^{00} e^{\alpha_{5} \beta(\Gamma-\chi) \eta p H} e^{\alpha_{5}(\Gamma-\chi) \gamma \phi_{f / s}^{0}}$, in which $\alpha_{1}, \alpha_{2}, \alpha_{3}, \alpha_{4}, \alpha_{5}$ are the transfer coefficients, and $k_{1}^{00}, k_{2}^{00}, k_{3}^{00}, k_{3}^{100}, k_{4}^{00}, k_{5}^{00}$ are the standard rate constants. The potential drop across the film/solution interface is $\phi_{f / s}=\phi_{f}-\phi_{s}=\alpha V+\beta p H+\phi_{f / s}^{0}$ and that across the metal/film interface is $\phi_{m / f}=\phi_{m}-\phi_{s}=(1-\alpha) V-\varepsilon L-\beta p H-\phi_{f / s}^{0}$, in which $\alpha=\frac{d \phi_{f / s}}{d V}$ is the dependence of the potential drop across the film/solution interface on $V$, $\beta=\frac{d \phi_{f / s}}{d p H}$ is the dependence of the potential drop across the film/solution interface on $p H, \phi_{f / s}^{0}$ is the value of $\phi_{f / s}$ at standard state; $\gamma=\frac{F}{R T}, \quad K=\varepsilon \gamma=\frac{\varepsilon F}{R T}$, $\varepsilon=1.10 \times 10^{6} \mathrm{~V} / \mathrm{cm}$, and $\alpha=0.728$ for passive iron [3]. 
Typical experimental impedance spectra for passive iron are shown in Figure III-1.2, in which real and imaginary parts of the impedance are plotted as a function of the measurement frequency with 20 data points evenly spaced in each frequency decade. In the high frequency range, real and imaginary parts of the impedance are almost independent of the measurement frequency and exhibit very small absolute values. DataFit software (version 7.1) was employed in this work in order to obtain the transfer coefficient $\alpha_{i}$ and standard rate constant $k_{i}^{00}$ for the $i$-th elementary interfacial reaction via nonlinear regression of experimental data, based on the derivation of an impedance model from the PDM. The values of $\beta$ and $\phi_{f / s}^{0}$ were also generated for passive iron through this method. The optimization algorithm depends on finding the minimum residual sum of squares between experimental data and simulated data. Details regarding the nonlinear regression procedure are described in the next section for a typical analysis of impedance data. Fundamental parameters for passive iron are presented in Table III1.2 and a comparison between experimental data and simulated data is displayed in Figure III-1.3. 


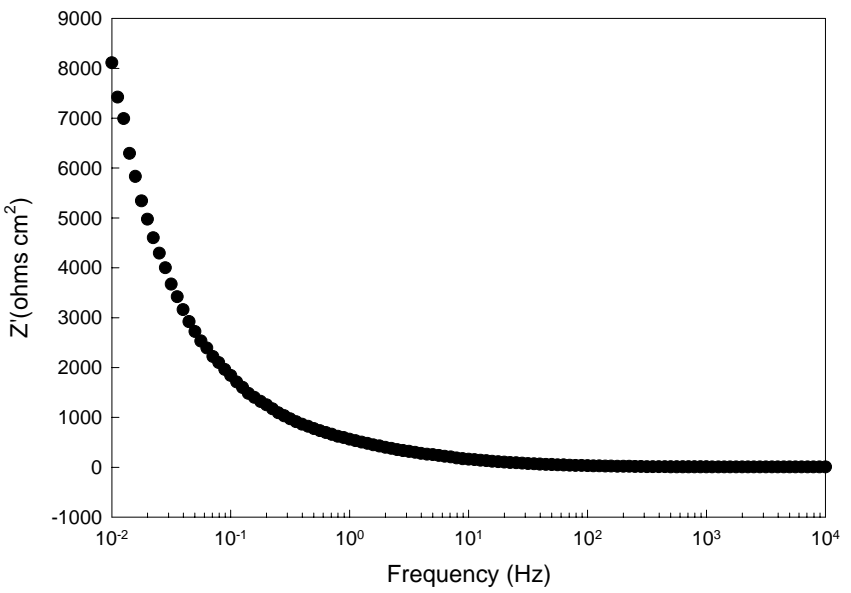

(a)

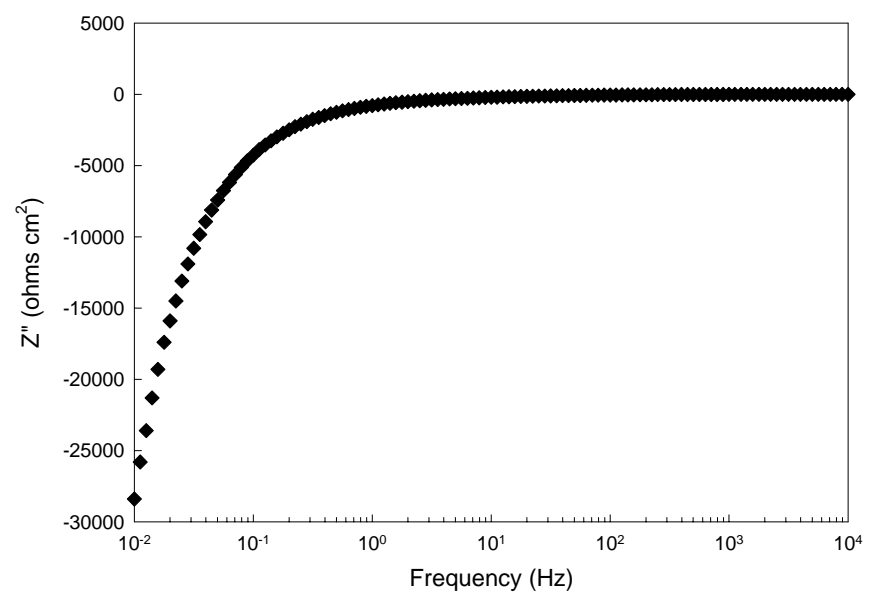

(b)

Figure III-1.2. Real part (a) and imaginary part (b) of impedance data for the passive film formed on iron in borate buffer solution with $0.01 \mathrm{M}$ EDTA $(\mathrm{pH} \mathrm{8.15)}$ at an applied film formation voltage of $0.2 \mathrm{~V}$ vs. SCE, plotted as a function of experimental frequencies ranging from $10^{-2} \mathrm{~Hz}$ to $10^{4} \mathrm{~Hz}$.

Table III-1.2. Fundamental parameters for the passive film formed on iron. 


\begin{tabular}{|l|l|l|}
\hline Parameter & Room Temp. & $75 \mathrm{C}$ \\
\hline$\alpha_{1}$ & 0.01 (from regression) & 0.0007 (from regression) \\
\hline$\alpha_{2}$ & 0.24 (from regression) & 0.06 (from regression) \\
\hline$\alpha_{3}$ & 0.39 (from regression) & 0.45 (from regression) \\
\hline$\alpha_{5}$ & 0.30 (from regression) & 0.12 (from regression) \\
\hline$k_{1}^{00}\left(\mathrm{~mol} \cdot \mathrm{cm}^{-2} \cdot \mathrm{s}^{-1}\right)$ & $3.8 \times 10^{-12}$ (from regression) & $9.9 \times 10^{-10}$ (from regression) \\
\hline$k_{2}^{00}\left(\mathrm{~mol} \cdot \mathrm{cm}^{-2} \cdot \mathrm{s}^{-1}\right)$ & $1.1 \times 10^{-15}$ (from regression) & $1.8 \times 10^{-20}$ (from regression) \\
\hline$k_{3}^{00}\left(\mathrm{~s}^{-1}\right)$ & $2.4 \times 10^{-6}$ (from regression) & $3.85 \times 10^{-7}$ (from regression) \\
\hline$k_{5}^{00}\left(\mathrm{~mol} \mathrm{O}^{.4} \cdot \mathrm{cm}^{-0.2} \cdot \mathrm{s}^{-1}\right)$ & $3.3 \times 10^{-8}$ (from regression) & $4.61 \times 10^{-8}$ (from regression) \\
\hline$\beta$ & -0.0047 (from regression) & .0002 (from regression) \\
\hline$\phi_{f / s}^{0}(\mathrm{Vvs} . \mathrm{SCE})$ & -0.29 (from regression) & -.007 (from regression) \\
\hline$\hat{\varepsilon}$ & 30 (from Reference [3]) & 30 (from Reference [3]) \\
\hline$\varepsilon(\mathrm{V} / \mathrm{cm})$ & $1.10 \times 10^{6} \quad$ (from Reference & $1.10 \times 10^{6} \quad$ (from Reference \\
& $[12])$ & $[12])$ \\
\hline$\alpha$ & 0.728 (from Reference [12]) & 0.728 (from Reference [12]) \\
\hline
\end{tabular}




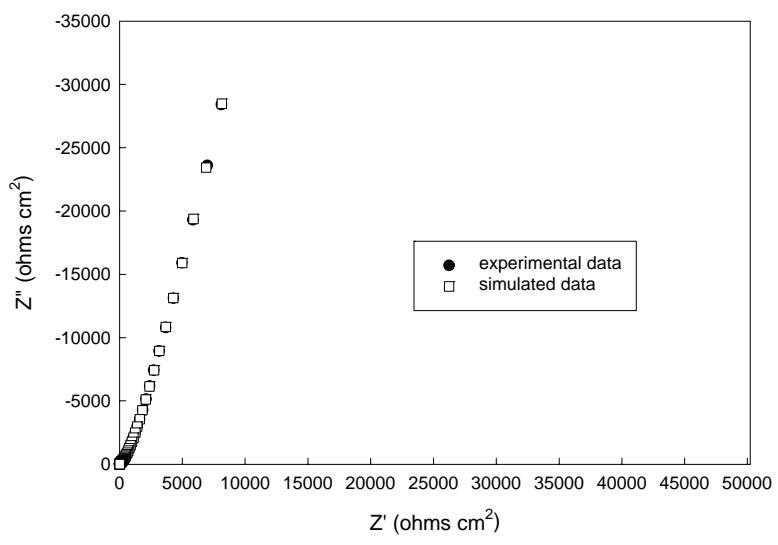

(a)

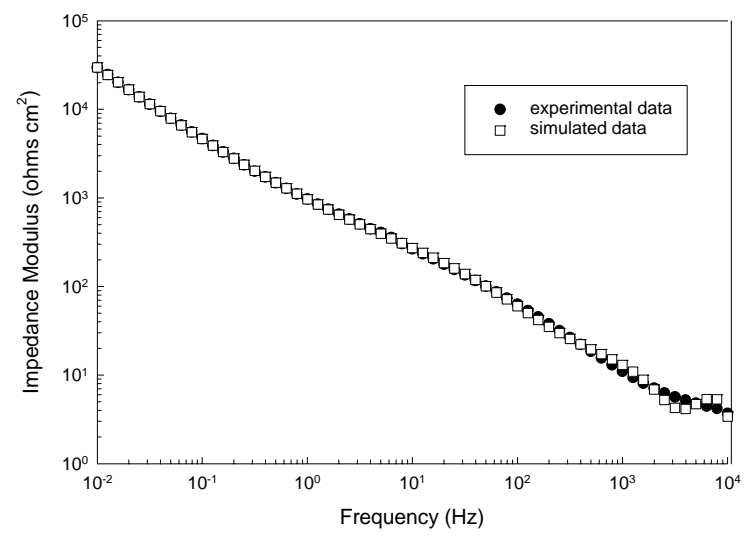

(b)

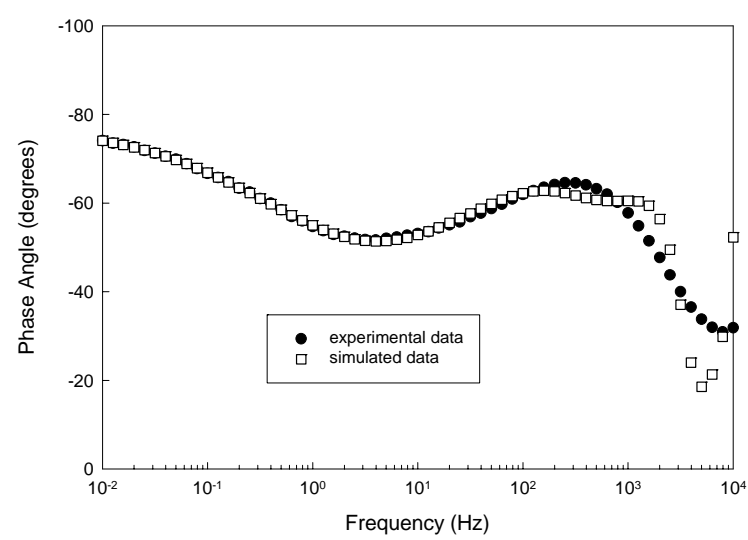

(c)

Figure III-1.3. Nyquist plot (a) and Bode plots (b, c) of impedance data for the passive film formed on iron in borate buffer solution with $0.01 \mathrm{M}$ EDTA $(\mathrm{pH} 8.15)$ at an applied film formation voltage of $0.2 \mathrm{~V}$ vs. SCE. Closed circles represent experimental data and open squares represent simulated data using nonlinear fitted parameters. 
The correlation between experimental impedance data and simulated data is good for Nyquist plots and modulus Bode plots in most cases. This validates the impedance model based on the PDM, through which a single set of parameters for passive iron (shown in Table III-1.2) have been derived via nonlinear regression in order to obtain simulated impedance data. However, the phase angle Bode plot displays some difference between experimental data and simulated data, especially in higher frequency range. A possible reason is that the phase angle, defined in Equation (7) as $\theta=\tan ^{-1} Z^{\prime \prime} / Z^{\prime}$, is a very sensitive function of $Z^{\prime}$ and $Z^{\prime \prime}$, so that a slight deviation in $Z^{\prime}$ or $Z^{\prime \prime}$ could give rise to a large fluctuation in $\theta$, which has a value only between $0^{\circ}$ and $-90^{\circ}$ for valid electrochemical impedance data.

Table III-1.2 shows that the standard rate constant for the iron interstitial production reaction (Reaction (1) in Figure III-1.1) is higher than that of the oxygen vacancy production reaction (Reaction (2) in Figure III-1.1) by several orders of magnitude. This implies that the generation of iron interstitials at the metal/film interface is much easier, considering that Reaction (1) is a lattice conservative process while Reaction (2) is not. However, the transfer coefficient $\alpha_{1}$ of Reaction (1) is close to 0 , which indicates that the intermediate activated complex for the charge transfer reaction (Reaction (1)) is very similar to the reactant $F e$, rather than the product $\mathrm{Fe}_{i}^{\chi+}$. Such observations are in agreement with the findings that that the dominant defects in the passive film formed on iron are oxygen vacancies, or iron interstitials, or both, due to the $\mathrm{n}$-type electronic properties. The value of $\beta$ is found to be -0.0047 , which is very close to that found in previous work [3], suggesting that $\beta$ has a value of 0 for the passive film formed on iron in borate buffer solutions.

Some regression analysis has been performed on the data taken at $75^{\circ} \mathrm{C}$. The results are shown in the third column of Table III-1.2. The results represent the fit for one set of data, however, fits will be made to two more sets of data to ensure that the parameters are in good agreement. It should be noted that the reaction rate constant for the injection of $\mathrm{Fe}_{i}^{\chi^{+}}$into the film is greatly increased (compared to room temp.), while 
the other reaction rate constants remain approximately the same or even decrease slightly. This was found to be the trend at all of the measured potentials.

It is well accepted that reaction rates are highly dependent on the temperature due to the relationship with the activation energy for a reaction. Despite this fact, it appears as if only one of the reaction rates increased by a substantial amount. This finding helps to explain the mechanism responsible for the higher steady state current observed at $75^{\circ}$ $\mathrm{C}$, which is $2.1 \pm 0.1 \mu \mathrm{A} / \mathrm{cm}^{2}$ (two orders of magnitude larger than that at room temp.). The information obtained here suggests that at higher temperatures, $\mathrm{Fe}_{i}^{\chi^{+}}$is being injected into the film much more rapidly and diffuses through the oxide layer much faster to account for the high steady state currents. These are the early findings on the temperature affects on the kinetics. Further analysis will be carried out to acquire more precise kinetic parameters.

The parameters in Table III-1.2 were used to calculate the steady-state properties for room temperature, including film thickness and current density, of passive films formed on iron. The results are shown in Figure III-1.4 and good agreement has been achieved between simulated data and experimental data from our previous research. This becomes another evidence for the validity of the PDM. Figure III-1.5 compares the thickness measured by ellipsometry to the thickness calculated through the assumption that the oxide film behaves as a parallel plate capacitor. It should be noted that a slight difference in thickness between figure III-1.4 and figure III-1.5. Figure III-1.4 displays the data obtained by earlier workers on this project, while figure III-1.5 shows more recent data. Although the thickness differs slightly, the slopes are nearly identical for room temperature. In addition, figure III-1.5 shows the thickness values as a function of formation voltage for films grown at $75^{\circ} \mathrm{C}$ with all other conditions being constant. Further regression will be carried out on the data to obtain the parameters and comparisons of the simulated data to the experimental data will be made. This analysis will be performed for $3 \mathrm{pH}$ 's, 3 temperatures, and 3 formations voltages to ensure that good agreement is made between the experimental and simulated data.

Issues and Concerns: $\quad$ The equations derived from PDM to describe the film under steady-state conditions do not account for the diffusivities of the various species in 
the film. The derivation approach used to obtain these equations clearly shows that the diffusion coefficients "drop out" when the equations are derived using the boundary conditions under steady state conditions. While there is no evidence of a mistake in this approach, we would intuitively expect that the diffusivities should play a role in the film's impedance. For this reason, we are currently re-deriving the equations using the Laplace Transform method.

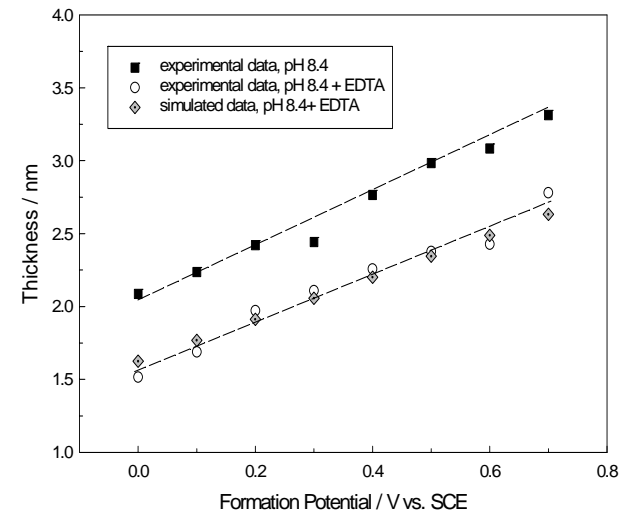

(a)

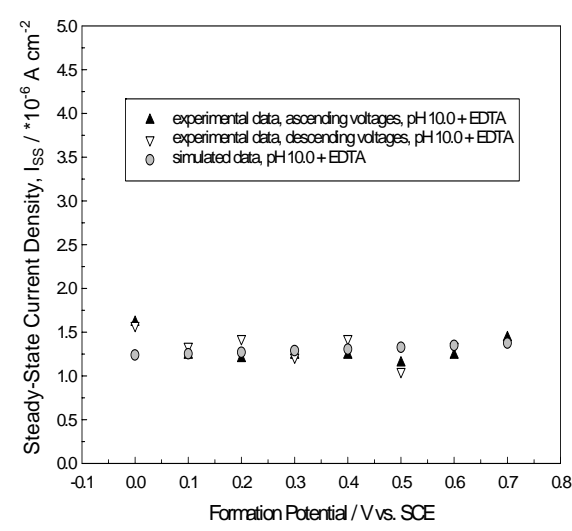

(b)

Figure III-1.4. The steady-state film thickness (a) and current density (b) for the passive film on iron. Simulated data were calculated using fundamental parameters in the Point Defect Model. 


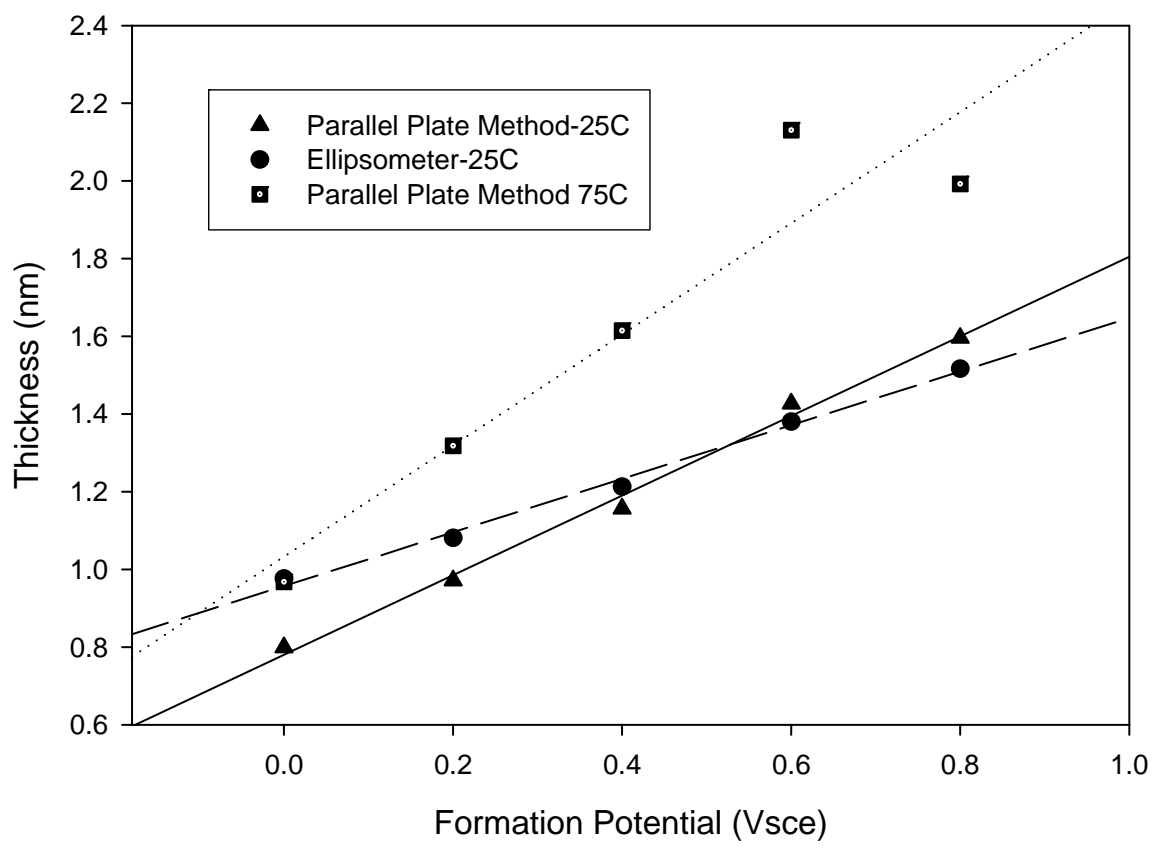

Figure III-1.5. The film thickness (as a function of formation potential) at room temperature as measured by both the parallel plate capacitance method and by the ellipsometer. The film thickness at $75^{\circ} \mathrm{C}$ is also shown.

\section{III-1.1c. XPS Work on Iron Oxide}

To begin, a spectrum analysis of the native oxide on iron was made using the XPS at the Penn State MRI laboratory. The sample appeared to contain the C 1s peak. To account for any shifts in the binding energy, the spectrums were calibrated using the $\mathrm{C} 1 \mathrm{~s}$ peak. Since the thickness of the iron oxide layer was desired for this sample, the peaks corresponding to iron were identified. As can be seen in Figure III-1.6 (blown up region of $\mathrm{Fe} 2 \mathrm{p}$ region), there was a splitting in the $\mathrm{Fe} 2 \mathrm{p}_{3 / 2}$ and was shifted slightly from the typical binding energy (BE) value of $707 \mathrm{eV}$ for iron. This shift (and splitting) can be attributed to the presence of both elemental and ionized iron. By using curve fitting software, the $\mathrm{Fe} 2 \mathrm{p}_{3 / 2}$ peak was convoluted into three curves representing the $\mathrm{Fe}^{\mathrm{o}}, \mathrm{Fe}^{2+}$, and $\mathrm{Fe}^{3+}$. The combination of the three curves yield the $\mathrm{Fe} 2 \mathrm{p}_{3 / 2}$ peak shown in figure III1.6. Next, by normalizing the integrated area below the peak, the relative amounts of the different Fe species were calculated. Table III-1.3 lists the values for the two runs made 
on the sample. The values were then substituted into equation 30 , where $\lambda_{\mathrm{Fe} 2 \mathrm{p}}$ (maximum escape depth) was assumed to be approximately 15 angstroms and $\mathrm{K}=2$ (sensitivity factor for iron). The "I" in equation 30 is the intensity of the recorded signal (subscript denotes the species) and the fraction represents the fractional amount of iron present, either in the ionic state in the oxide $(\mathrm{FeO})$ or as pure iron in bulk. From this equation, the thickness was calculated to be somewhere between 19-27 angstroms, which agrees with values obtained in the literature.

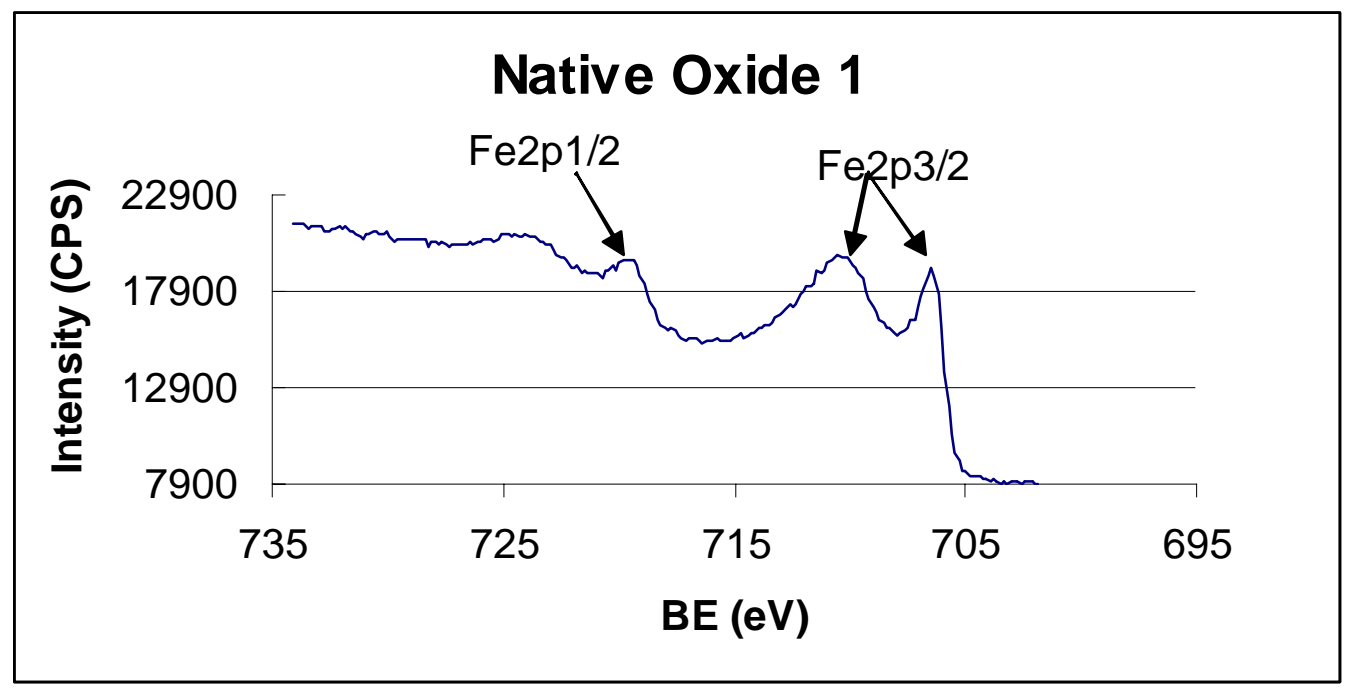

Figure III-1.6. XPS spectrum in the Fe2p region. Notice the splitting of the $\mathrm{Fe} 2 \mathrm{p} 3 / 2$ peak.

Table III-1.3. Table displaying relative amounts of iron species present in Fe 2p3/2 peak. The total amount of ionized iron present (used in equation 1) is found by adding the relative amounts of $\mathrm{Fe}^{2+}+\mathrm{Fe}^{3+}$.

\begin{tabular}{|l|l|l|l|}
\hline Species Present & Integrated Area for Run 1 & \multicolumn{2}{|l|}{ Relative Amounts Present } \\
\cline { 3 - 4 } & & Run 1 & Run 2 \\
\hline $\mathrm{Fe}^{\mathrm{O}}$ & 1413.7 & $28 \%$ & $43.3 \%$ \\
\hline $\mathrm{Fe}^{2+}$ & 710.0 & $49 \%$ & $41.7 \%$ \\
\hline $\mathrm{Fe}^{3+}$ & 712.0 & $23 \%$ & $15 \%$ \\
\hline
\end{tabular}

thickness $=t=\lambda_{\mathrm{Fe} 2 p} * \ln \left[K \frac{I_{\mathrm{FeO}}}{I_{\mathrm{Fe}}}+1\right]$

So far, measurements have been performed on samples with oxide layers grown in borate buffer solutions (containing EDTA) at three potentials in the passive range. Analysis has only been performed on the native oxide layer to verify if this method will 
be useful. It shows the thickness to be in good agreement with other types of measurements. If this method provides the relative amounts of iron cations $(2+$ or $3+)$, the information can be incorporated into the Curve Fitting Algorithm to better account for the charged species present in the oxide layer.

Issues and Concerns: The data quite obviously shows the presence of both atomic iron and iron cations existing near the surface. Because the ionization levels of $\mathrm{Fe}^{2+}$ and $\mathrm{Fe}^{3+}$ are very close, it is somewhat difficult to differentiate between the two ionized species. However, the shapes of the peaks strongly indicate the presence of more than one oxidation state. Therefore, a thorough analysis of the data should allow us to distinguish between the two species to a certain degree of accuracy. In addition, we will analyze the concentration of oxygen as a function of depth beneath the surface. This will provide further clues as to the oxidation states of iron and the oxygen vacancy concentrations as a function of distance across the film.

\section{III-1.2 Obtaining Kinetic Information for Passive Film Growth via Curve Fitting}

This section describes the algorithm used in Data Fit to fit the derived impedance equation to the data. Fitting the data to the equation yields the values for the kinetic parameters, which are variables in the algorithm. The code for the impedance algorithm is provided below. There are nine equations (conditions) with ten unknowns (variables). A list displaying what each condition and variable represents is shown below. It is through this algorithm that the kinetic parameters in table III-1.2 are extracted.

$$
\begin{aligned}
& \mathrm{F} 1=\mathrm{x} 1+0.241 \\
& \mathrm{~F} 2=\mathrm{x} 2 \\
& \mathrm{~F} 3=(28.2474 * \mathrm{~m} 2-9.45042 * \mathrm{~m} 5) /(114236000 * \mathrm{~m} 2) * \mathrm{~F} 1- \\
& 1 /(114236000 * \mathrm{~m} 2) * \log \left((\mathrm{k} 50 * \exp (\mathrm{m} 5 / 3 * 38.9441 *(\mathrm{p} 1 * \mathrm{~F} 2+\mathrm{p} 2))) /\left(\mathrm{k} 20^{*} \exp (-\right.\right. \\
& \left.\mathrm{m} 2 * 8.0 / 3 * 38.9441 *(\mathrm{p} 1 * \mathrm{~F} 2+\mathrm{p} 2))) *\left(10^{\wedge}(-\mathrm{F} 2)\right)^{\wedge} 0.6\right)
\end{aligned}
$$




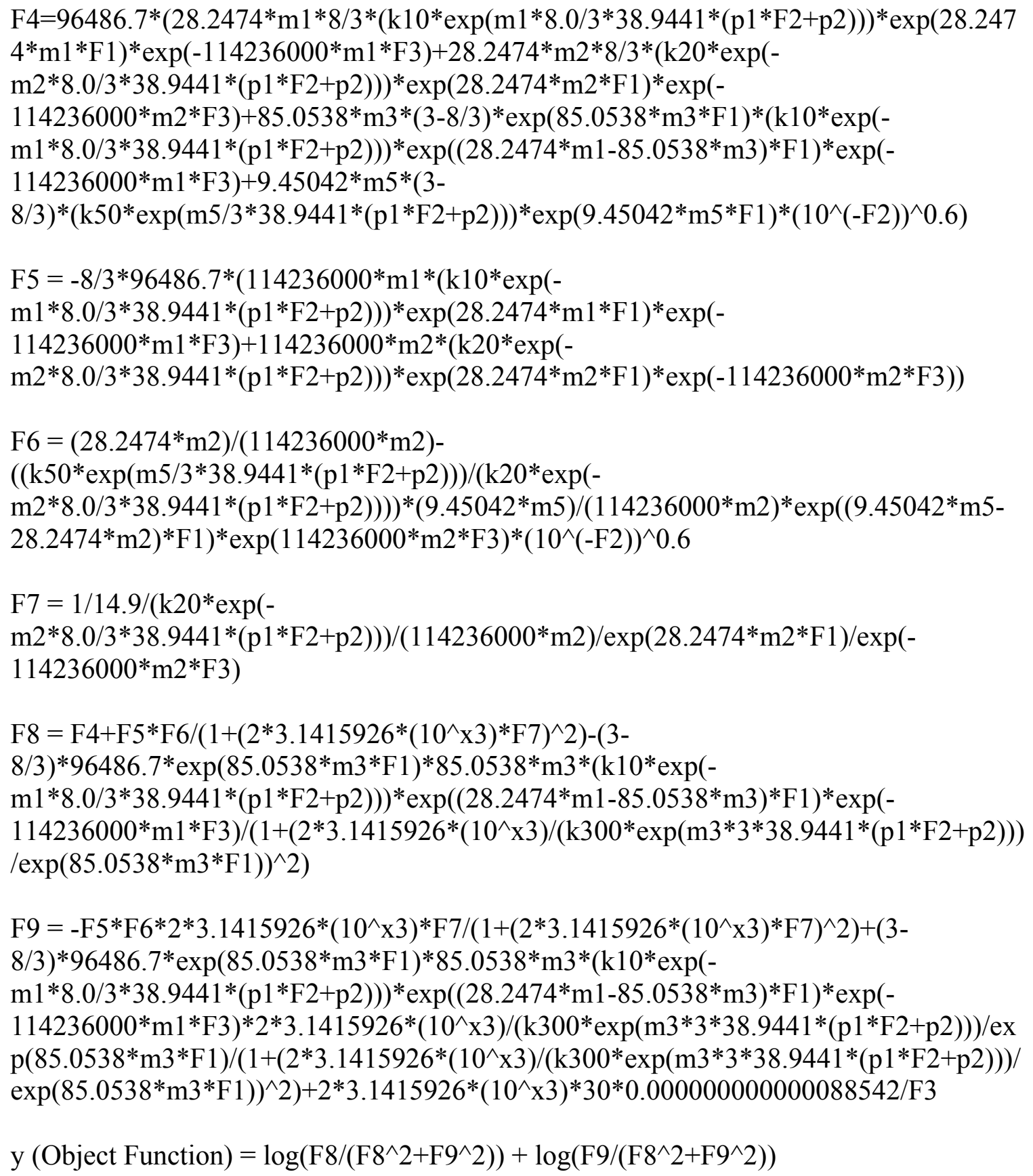

\section{Conditions:}

$\mathrm{F} 1=$ potential $(\mathrm{SHE}$ scale $)$

$\mathrm{F} 2=\mathrm{pH}$

$\mathrm{F} 3=$ film thickness equation

$\mathrm{F} 4=\partial \mathrm{I} / \partial \mathrm{V}$

$\mathrm{F} 5=\partial \mathrm{I} / \partial \mathrm{L}$ 
$\mathrm{F} 6=\phi_{2}$ (equation 10 above)

$\mathrm{F} 7=\tau_{2}$ (equation 11 above)

F8 = real component of the admittance

F9 = imaginary component of the admittance

$\mathrm{Y}=\mathrm{y}($ object function $)=$ real/imaginary component of the impedance (the equation for the real component is shown above, simply replace (F8) by $(-\mathrm{F} 9)$ in the numerator for the imaginary component)

Variables:

$\mathrm{m} 1=\alpha_{1}$

$\mathrm{m} 2=\alpha_{2}$

$\mathrm{m} 3=\alpha_{3}$

$\mathrm{m} 5=\alpha_{5}$

$\mathrm{k} 100=$ standard rate constant for reaction 1

$\mathrm{k} 200=$ standard rate constant for reaction 2 $\mathrm{k} 300=$ standard rate constant for reaction 3 $\mathrm{k} 500=$ standard rate constant for reaction 5

$\mathrm{p} 1=\beta$

$\mathrm{p} 2=\phi^{\circ}$

$\mathrm{m}=\alpha$

$\mathrm{e}=\varepsilon=$ electric field strength in the film e1 $=$ dielectric constant for iron oxide.

Issues and Concerns: $\quad$ The magnitude of the relative error in some of the fits is quite large at high frequencies. We found that this is most likely due to the high number of parameters being used to fit the equation ( 9 parameters). The high number of parameters can give rise to a very large number of "solutions" or "fits". For this reason, we are going to use experimental techniques to measure the film dissolution rate. From this rate, we can calculate two of the rate constants used to fit the equation to the data. We are researching methods to measure some of the other parameters in hopes of decreasing the number of "solutions" to the problem. This should provide us with information to allow for much more accurate fits in the high frequency regions. We are also looking into another data fitting software package that uses different fitting algorithms than the more popular commercially available ones. We hope to find that these different algorithms will allow better fitting. 


\section{III-1.3 Experimental Setup to Obtain Data for DFA}

The development of the Damage Function Analysis (DFA) is described in detail in another section of this report. Only the experimental set-up for obtaining data to be used in the development of DFA is being described here. However, it should be mentioned that the original DFA requires data for the number of pits/area as a function of the depth below the surface of the sample [2]. This view comes from the idea that pits nucleate and grow at different times and rates and the pits can either be active (growing) or dead (repassivated). Because pits nucleate and grow at different times and rates, there should be a trend showing the number of pits/area decreases with depth. If the number of pits/area surpasses a critical value at a particular depth, failure can occur. By using data on the number of pits/area as a function of depth and time, DFA can predict failure due to pitting corrosion.

Taking this perspective can lead to some problems as depicted in figure III-1.7. It can easily be seen that the number of pits/area at the various depths does not definitively follow a trend where there is a higher density of pits at the surface which decreases with depth below the surface. This apparent inconsistency arises due to the fact that pits grow in complex shapes as the pit front proceeds deeper beneath the surface. To rectify this problem, it has been suggested that the DFA account for the depth of the deepest pit as function of time, temperature, and aggressive ion concentration. 


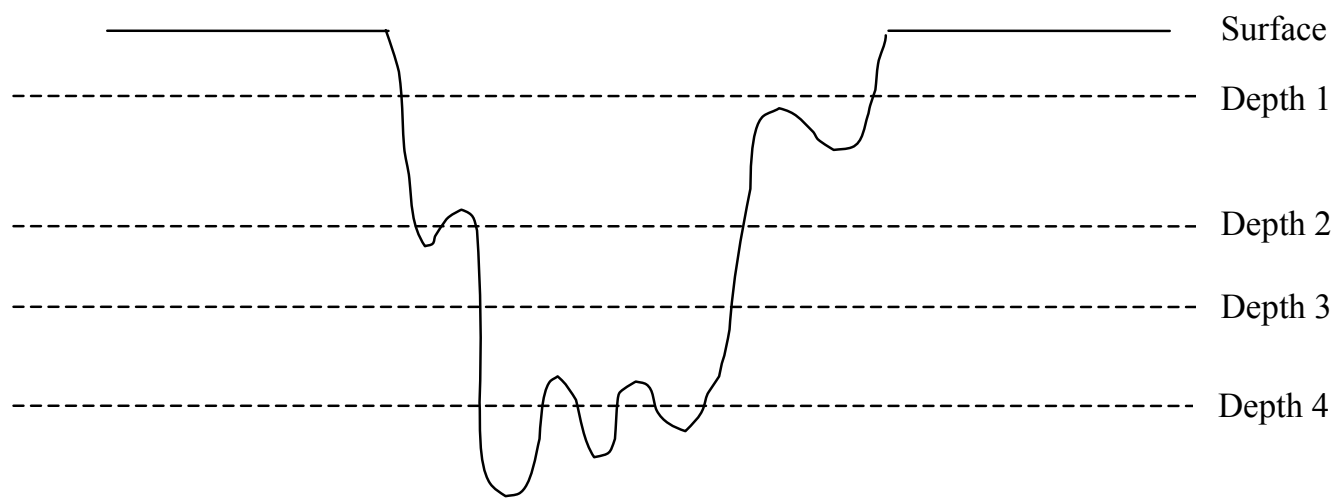

Figure III-1.7. Due to the complex shape of a pit, the number of pits/area as a function of depth does not appear to decrease as would be expected. Depth 1 shows 1 pit, depth 2 shows 2 pits, depth 3 shows 1 pit, and depth 4 shows 3 pits.

To obtain this data, ASTM A516 steel will be placed in solutions for various times-1 week, 2 week, 1 month, and 4 month times have been suggested. Three temperatures $\left(25^{\circ} \mathrm{C}\right.$ to $90^{\circ} \mathrm{C}$ ), potentials (from the corrosion potential to the upper end of the passive region), $\mathrm{pH}$ 's (from 7 to 14), and aggressive ion concentrations will be analyzed. For each condition, nine samples will be used to obtain the data. These samples can be measured simultaneously. After the set time period, the samples will be removed from the solution and the maximum pit depth for each of the nine samples will be measured. This information will then be used as the input data for the DFA as discussed in the DFA section of this report.

Based on Extreme Value Statistics, DFA can predict damage/failure due to pitting corrosion. This is a statistical approach based on empirical data. However, by assuming that vacancy coalescence in the film is the mechanism responsible for pit nucleation [5], a deterministic-probabilistic DFA can be developed. The terms describing the vacancy coalescence have been derived and are based on the kinetic parameters extracted from the PDM. Applying the kinetic parameters from the experiments on iron, the critical vacancy density for pit formation can be calculated. This information, if shown to be correct, can prove to be a monumental find in the understanding of pitting corrosion. 
Issues and Concerns: $\quad$ None

\section{III-1 References}

6. D.D. Macdonald, M. Uriquidi-Macdonald, J. of Electrochemical Society, 137, 2395 (1990).

7. G. Engalhardt, D.D. Macdonald, Corrosion, 54, 469 (1998).

8. J. Liu, D.D. Macdonald, J. of Electrochemical Society, 148, B425 (2001).

9. D. D. Macdonald, Pure Appl. Chem., 71, 951 (1999).

10. D.D. Macdonald, J. of Electrochemical Society, 139, 3434 (1992).

\section{III-2. Task: The Role of Chloride Ion in Passivity Breakdown on Nickel}

\section{$\underline{\text { Task Status }}$}

\section{III-2.1 Experimental set up}

All electrochemical studies were carried out in a three-electrode, PTFE electrochemical cell. A platinum counter electrode and a saturated calomel reference electrode (SCE) were used to carry the cell current and to measure the potential of the working electrode, respectively. All electrode potentials are referred to the SCE scale. Pure Ni (99.99 \%, Alfa Aesar) was used as the working electrode. The nickel rod was mounted in epoxy resin with one end of $1-\mathrm{cm}^{2}$ area being exposed to the solution. Prior to performing an experiment, the exposed nickel was polished with successively finer $\mathrm{SiC}$ papers ranging from 600 to 2000 grit. The solution used in this study was deaerated, $\mathrm{pH}=8.5$ borate buffer solution prepared by mixing $0.2 \mathrm{M}$ boric acid and $0.05 \mathrm{M}$ citric acid with $0.1 \mathrm{M}$ tertiary sodium phosphate solution [1]. The concentration of $\mathrm{Cl}^{-}$ion was established by adding the requisite amount of $\mathrm{NaCl}$ to the solution. All experiments were performed at ambient temperature $\left(22 \pm 2{ }^{\circ} \mathrm{C}\right)$. The working electrode was initially reduced potentiostatically at $-1.0 \mathrm{~V}_{\mathrm{SCE}}$ for $30 \mathrm{~min}$. to remove any air-formed oxide on the surface, and then a passive film was grown potentiodynamically by ramping the applied potential from $E_{\text {corr }}$ to $400 \mathrm{mV}_{\mathrm{SCE}}$ at a sweep rate of $0.5 \mathrm{mV} / \mathrm{sec}$. After reaching 400 $\mathrm{mV}_{\mathrm{SCE}}$, that potential was maintained for 2 hours before initiating the measurements of interest. 
Electrochemical experiments were performed using a Solartron 1287 Electrochemical Interface. Electrochemical impedance data were recorded with a Solartron 1255B Frequency Response Analyzer (FRA), using an excitation voltage of 10 $\mathrm{mV}$ (peak-to-peak). Capacitance data for Mott-Schottky analysis were obtained at constant frequency of $1 \mathrm{kHz}$.

Issues and Concerns: $\quad$ None

\section{III-2.2 Applying Mott-Schottky Analysis and EIS to Study Passivity Breakdown}

The polarization behavior of nickel in the borate buffer solution with or without chloride present is shown in Figure III-2.1. Chloride is seen to induce passivity breakdown at progressively lower potentials as the chloride concentration increases. The voltage at which the measurements were made in this study $\left(400 \mathrm{mV}_{\mathrm{SCE}}\right)$ is about $50 \mathrm{mV}$ more negative that the apparent breakdown voltage for the most concentrated chloride solution. 


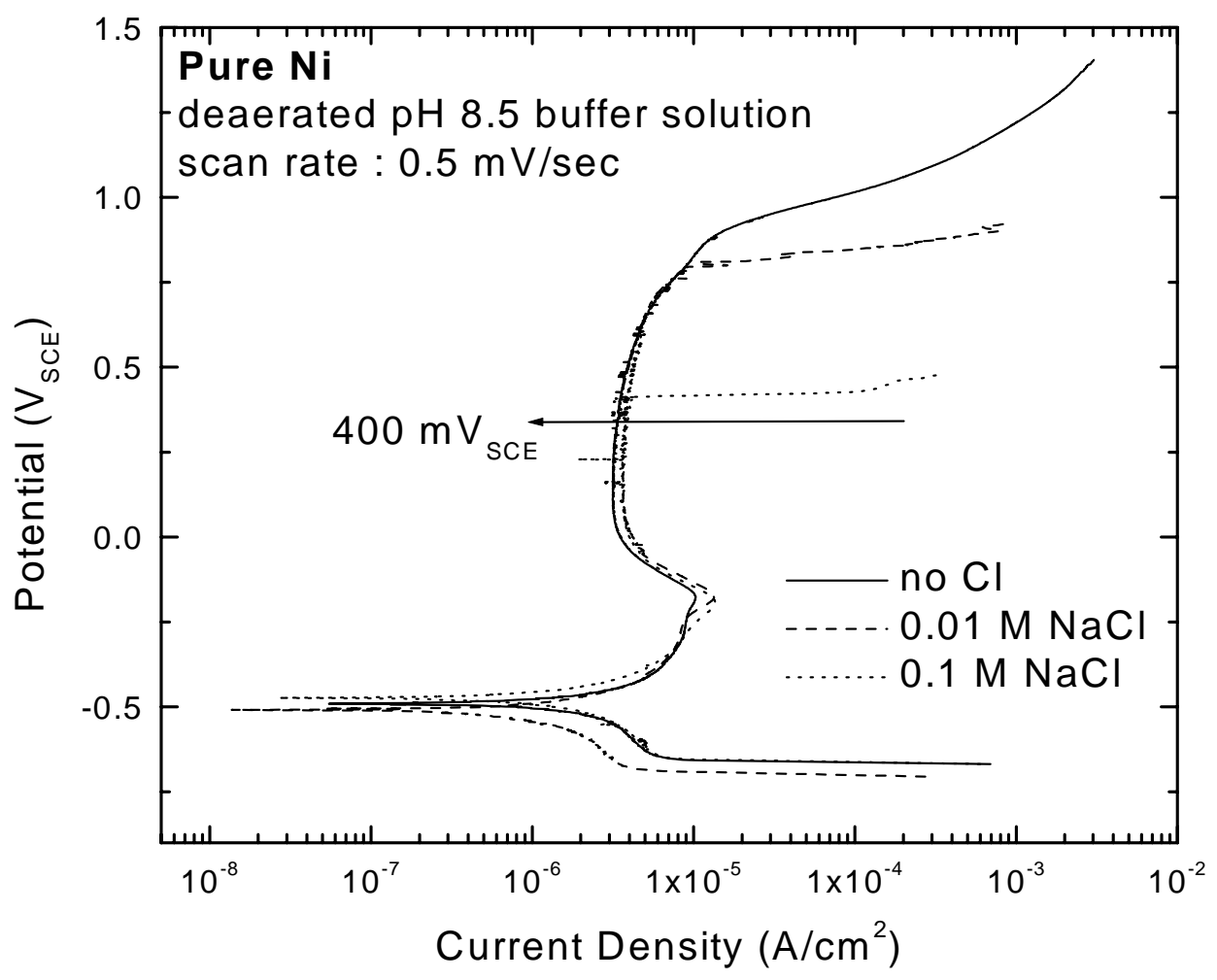

Figure III-2.1 Polarization curves for nickel in borate buffer solution with or without chloride ion.

Figure III-2.2 shows the effects of $\left[\mathrm{Cl}^{-}\right]$on potentiostatic transients (chronoamperograms) for $\mathrm{Ni}$ obtained by stepping the potential from the open circuit value to $400 \mathrm{mV}_{\mathrm{SCE}}$. The transients are typical in form, in that the current decreases with time. At any given time after imposition of the potential step, the passive current density was found to be slightly greater with increasing chloride concentration. Thus, chloride ion catalyzes the transfer of charge across the passive film, but it is not a strong effect. It is notable that meta-stable pitting events are observed for the specimen immersed in the solution containing $0.1 \mathrm{M} \mathrm{NaCl}$, indicating that passivity breakdown occurs under the prevailing conditions. 


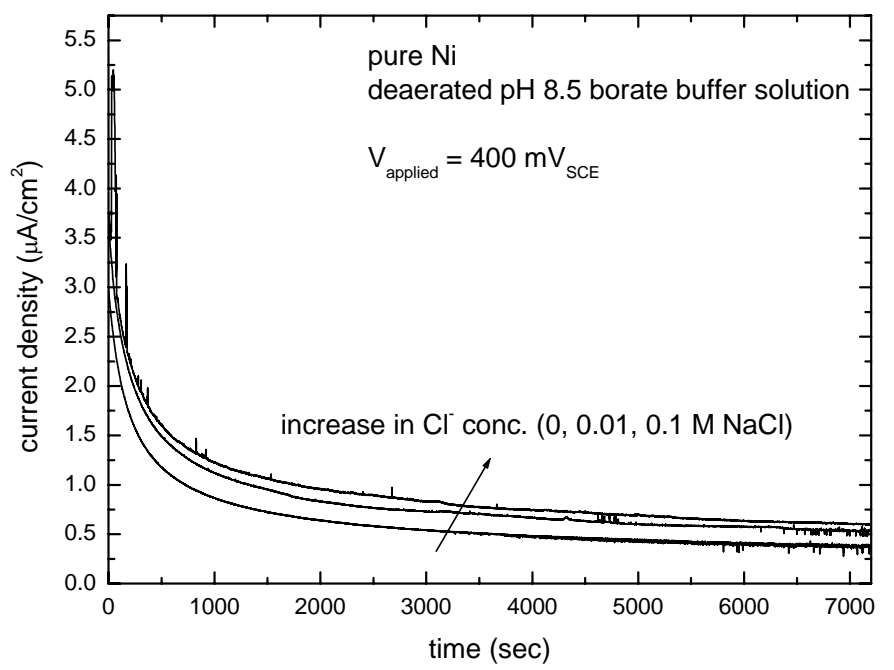

Figure III-2.2 Current transient curves of $\mathrm{Ni}$ in chloride-containing, deaerated, $\mathrm{pH} 8.5$ borate buffer solutions measured at $400 \mathrm{mV}_{\mathrm{SCE}}$. The concentration of $\mathrm{Cl}^{-}$was controlled from 0 to $0.1 \mathrm{M}$ by adding $\mathrm{NaCl}$ to the solution.

From previous studies on the electronic properties of the passive film on $\mathrm{Ni}$ [2], the barrier layer was found to be $\mathrm{p}$-type semiconductor within the passive range, due to a preponderance of cation vacancies $\left(V_{N i}^{\chi^{\prime}}\right)$ in the lattice. The preponderance of cation vacancies over oxygen vacancies $\left(V_{o}^{*}\right)$ is rationalized by the fact that the energy of formation of $V_{N i}^{\chi}$ is smaller relative to that of $V_{o}^{\prime}$, when the oxidation state of the metal is small ( $\chi=2$ for $\mathrm{Ni}$ in the passive state) [3].

To investigate the effects of $\mathrm{Cl}^{-}$on the electronic properties of passive film, Mott-Schottky analyses were performed at a fixed frequency of $1 \mathrm{kHz}$. The interfacial capacitance, $C$, is obtained from $C=1 / \omega Z$, where $\omega$ is the angular frequency and $Z$ " is the imaginary part of impedance. Assuming that the capacitance of the Helmhotlz layer can be neglected (because it is much larger than the space charge capacitance of the passive film and the capacitances are in series), the measured capacitance $C$ is equal to the space charge capacitance, $C_{s c}$. According to Mott-Schottky theory [4] the space charge capacitance of a p-type semiconductor is given by Equation (1), 


$$
\frac{1}{C^{2}}=\frac{-2}{\varepsilon \varepsilon_{0} e N_{A}}\left(V-V_{f b}-\frac{k T}{e}\right)
$$

where $\varepsilon$ is the dielectric constant of the oxide, $\varepsilon_{0}$ is the vacuum permittivity, $e$ is the charge of an electron, $N_{A}$ is the acceptor concentration in the film, $V$ is the applied potential, $V_{f b}$ is the flat band potential, and $k$ is the Boltzmann constant. Thus, for a ptype semiconductor, $C^{-2}$ versus $V$ should be linear with a negative slope that is inversely proportional to the acceptor concentration.

Figure III-2.3 shows the effects of $\mathrm{Cl}^{-}$on Mott-Schottky plots for passive nickel. The Mott-Schottky plots are characteristic of p-type semi-conducting passive film and the acceptor levels in the film, calculated from the slopes and using $\varepsilon=12[5,6]$, are presented in Figure III-2.4. It is clear that the acceptor level within the passive film increases with increasing $\mathrm{Cl}^{-}$concentration. Previously, Macdonald et al. [7] and Liu et al. [8] reported that there exists a correlation of high fidelity between the crystallographic defect structure and the electronic structure for passive films on pure metals, which shows that the defects (donors for n-type semiconductors and acceptors for p-type semiconductors) are themselves the dopants, including cation vacancies (p-type), anion vacancies (n-type), and cation interstitials (n-type). Accordingly, the acceptor species in Figure III-2.4 are considered to be cation vacancies $\left(V_{N i}^{\chi^{\prime}}\right)$ in the passive film. These results demonstrate that $\mathrm{Cl}^{-}$contained in $\mathrm{pH} 8.5$ borate buffer solution increases the concentration of the metal vacancy in the passive film on Ni. However, it should be noted that chloride was present in the solution during the film formation period, and hence the defect structure may have been "built-in" during the growth process, rather than being induced by the interaction of chloride ion with the outer surface of the film. 


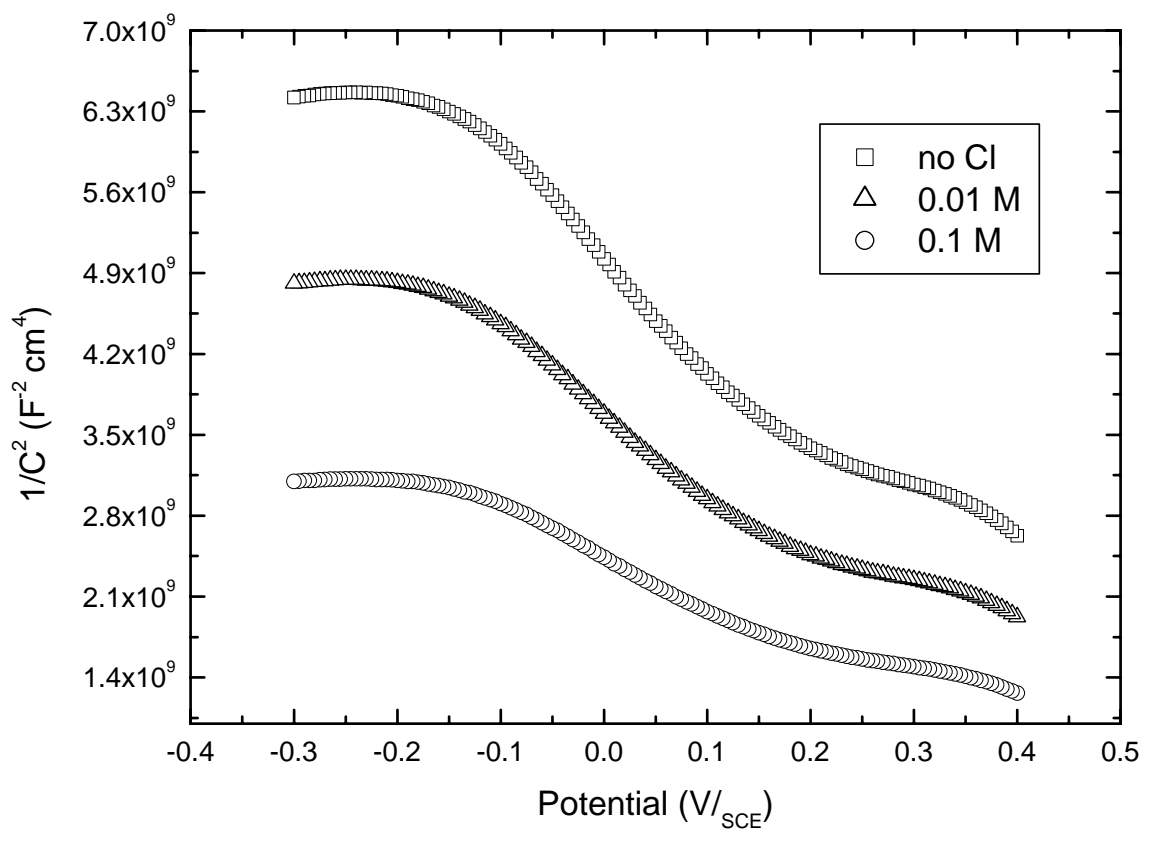

Figure III-2.3 Mott-Schottky plots for the passive film formed on $\mathrm{Ni}$ in deaerated, $\mathrm{pH} 8.5$ borate buffer solution at $400 \mathrm{mV}_{\mathrm{SCE}}$ for 2 hours with different concentration of $\mathrm{Cl}^{-}$ions measured at a frequency of $1 \mathrm{kHz}$. 




Figure III-2.4 Acceptor levels calculated from the slopes of the Mott-Schottky plots in Fig. 6.

To examine the effect of adding $\mathrm{Cl}^{-}$to the solution after passivity had been established in the absence of this species, another set of Mott-Schottky analyses were performed. Thus, the passive film was initially grown in $\mathrm{Cl}^{-}$-free, $\mathrm{pH} 8.5$ borate buffer solution at $400 \mathrm{mV}_{\mathrm{SCE}}$ for 2 hours. Then, $\mathrm{NaCl}$ was added to the solution to yield a chloride concentration of $0.1 \mathrm{M}$. Mott-Schottky plots were then obtained as a function of time (Figure III-2.5), and the calculated acceptor levels, shown in Figure III-2.6, were calculated. These data show that chloride induces an increase in the concentration of cation vacancies in the barrier layer after the film is formed. This finding is inconsistent with chloride modifying the film growth process, but it is consistent with the presence of a chloride-catalyzed cation vacancy generation process at the barrier layer/solution interface. The nature of that process is such that it results in a linear increase in the cation vacancy concentration with time, at least over the first four hours of exposure to chloride. We should note, that Mott-Schottky analysis presumably yields the acceptor 
concentration averaged over all sites on the surface. However, localized corrosion occurs at sites that occupy only a very small fraction of the total area, so that the level of enhancement of the cation vacancy concentration at the breakdown sites is presumably much greater than indicated in Figures III-2.4 and III-2.6.

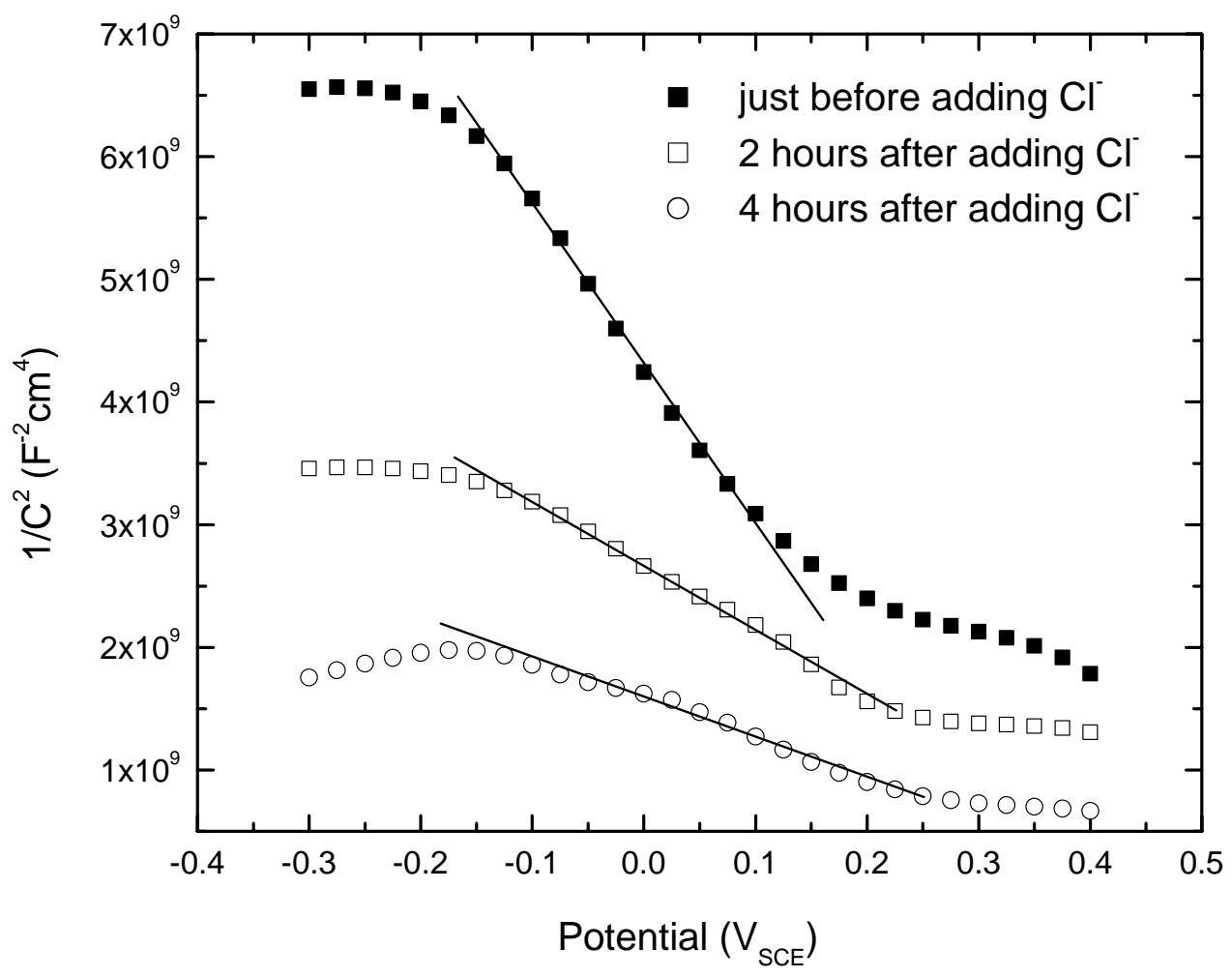

Figure III-2.5 Mott-Schottky plots for the passive film formed on Ni in deaerated, $\mathrm{pH} 8.5$ borate buffer solution at $400 \mathrm{mV}_{\mathrm{SCE}}$, as calculated from capacitance data measured at a frequency of $1 \mathrm{kHz}$. The passive film was initially grown in chloride-free solution for 2 hours and then $\mathrm{NaCl}$ was added to the solution to adjust the total concentration of $\mathrm{Cl}^{-}$to $0.1 \mathrm{M}$. 


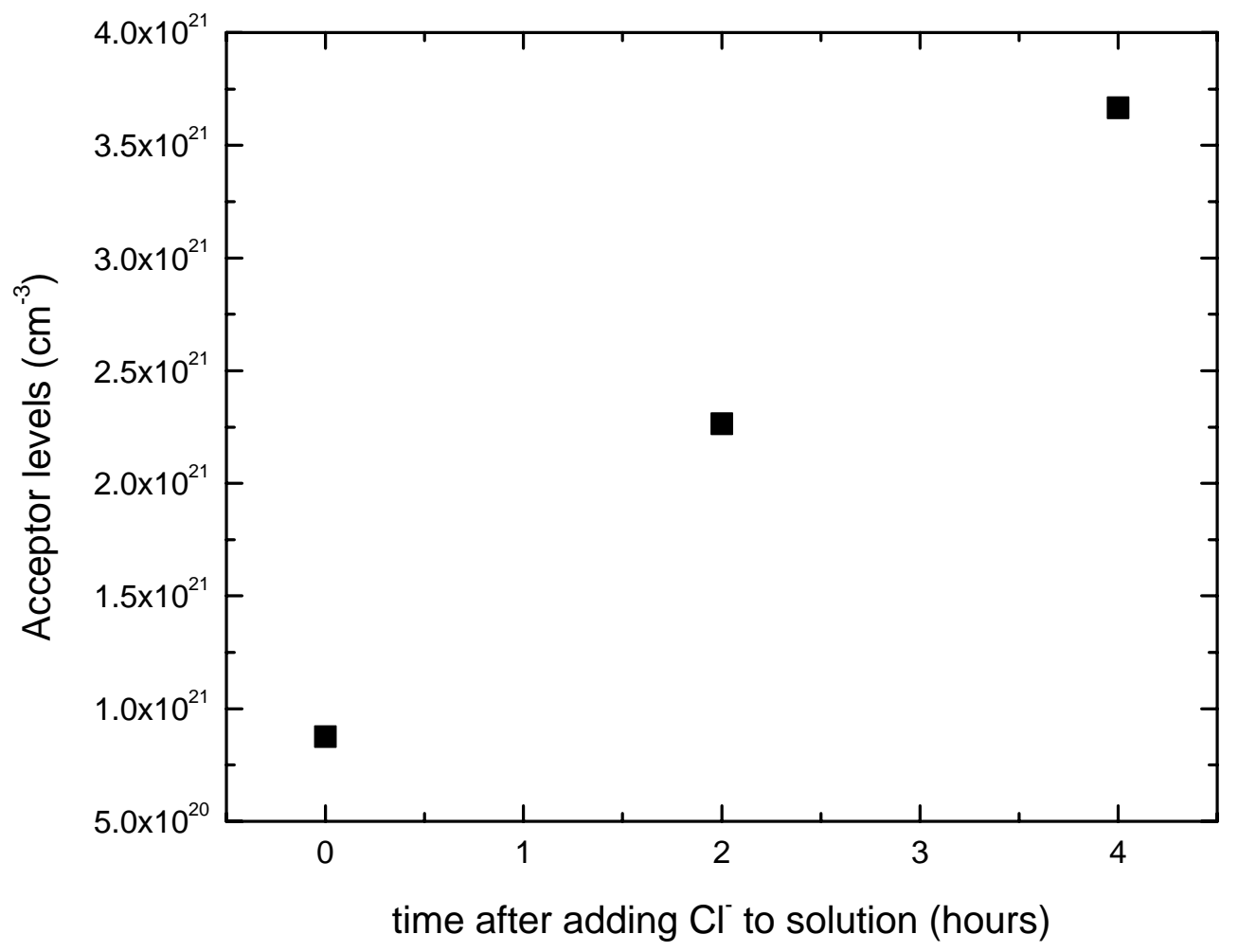

Figure III-2.6 Acceptor levels calculated from the slopes of the Mott-Schottky plots in Fig. 8.

The final issue that we wish to explore is the fundamental nature of the processes that occur at the barrier layer/solution interface that result in an increase in the cation vacancy concentration within the barrier layer, when the system is exposed to chloride ion. This issue was explored using electrochemical impedance spectroscopy (EIS) to measure the impedance of the $\mathrm{Ni} / \mathrm{NiO} /$ solution interphase over a wide frequency range and by then fitting a reduced PDM to the data, in order to extract values for important parameters in the model. The fitting was done using an optimization program from Oakdale Engineering, www.curvefitting.com. The reduced PDM was generated by ignoring reactions involving metal interstitials [Reactions 2 and 5, Figure II-2.1], because metal interstitials would dope the film n-type in electronic character and no hint of n-type behavior is observed at $400 \mathrm{mV}_{\text {SCE}}$. A full account of the fitting procedure will be 
published at a later date. The impedance data were found to correctly transform according to the Kramers-Kronig integral transforms, thereby demonstrating that the system conforms to the constraints of linear systems theory [9].

Figure III-2.7 shows experimental impedance data for passive $\mathrm{Ni}$, together with the model fit for the reduced PDM. The fit is judged to be very good for the three systems explored $(0,0.01$, and $0.1 \mathrm{M} \mathrm{NaCl})$. Values for selected parameters determined from the optimization are summarized in Table III-2.1. Of the parameters listed, only the electric field strength was assumed on the basis of previous work [10]; no credible reason exists within the PDM for believing the electric field strength across the barrier layer should depend upon the chloride concentration. Of the parameters listed, $k_{i}^{00}$ represents the standard rate constant and $\alpha_{i}$ is the transfer coefficient of $i$-th reaction in Figure II-2.1. Examination of the data in Table III-2.1 shows that there is no systematic dependence of any of the kinetic parameters listed in Table III-2.1 on chloride concentration, except for the rate constant for the cation ejection Reaction 4, Figure II-2.1. In this case, even though the experimental data are not extensive and assuming that the kinetic order must be an integer, the rate constant evidently increases in a first order fashion with chloride concentration (Figure III-2.8). Because this reaction is envisioned to be elementary in nature, the kinetic order is equivalent to the molecularity and hence we conclude that the reaction involves a single chloride species. This finding demonstrates unequivocally that the role of chloride in inducing passivity breakdown on nickel is catalysis of the cation vacancy generation reaction at the barrier layer/solution interface, as envisioned in the Point Defect Model (Figure II-2.3). 


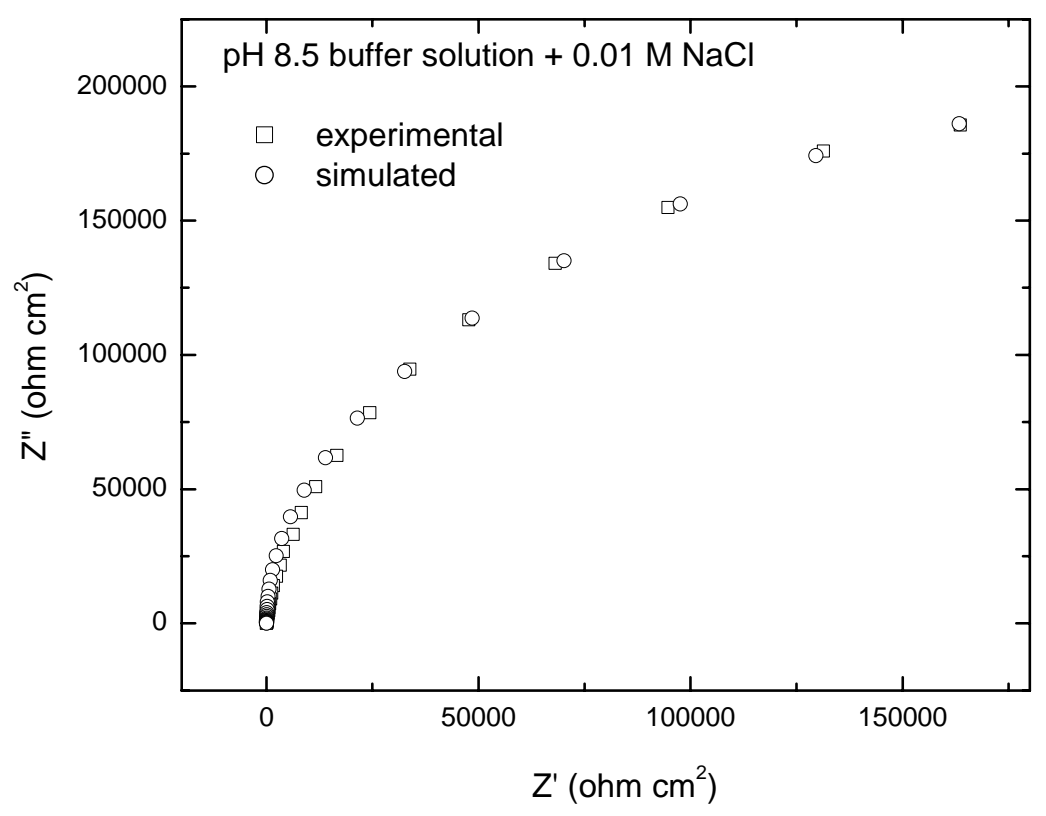

Figure III-2.7 Nyquist plot for the passive film formed on Ni in chloride-containing, $\mathrm{pH} 8.5$ borate buffer solutions at $400 \mathrm{mV}_{\mathrm{SCE}}$ for 2 hours, together with the model fit by the PDM. The concentration of $\mathrm{Cl}^{-}$in the solution was (a) $0 \mathrm{M}$, (b) $0.01 \mathrm{M}$ and (c) $0.1 \mathrm{M}$. 


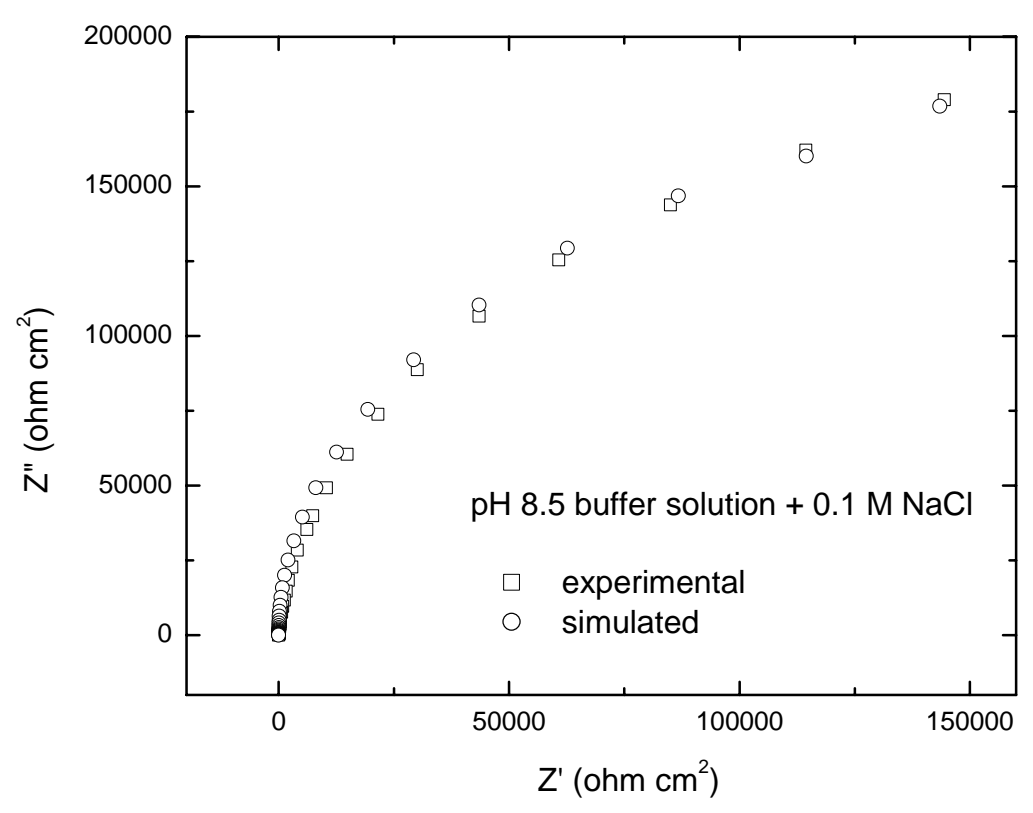

Figure III-2.7 Kinetic plot for cation vacancy generation at the barrier layer/solution interface (Reaction 4, Figure II-2.1). The line is drawn for a first order dependence of the rate of the reaction on chloride concentration while the points with error bars are experimental data.

Table III-2.1 Values for various parameters in the Point Defect Model, as determined by fitting the PDM to the experimental electrochemical impedance data.

\begin{tabular}{|c|c|c|c|}
\hline Parameter & $0 \mathrm{M} \mathrm{NaCl}$ & $0.01 \mathrm{M} \mathrm{NaCl}$ & $0.1 \mathrm{M} \mathrm{NaCl}$ \\
\hline Electric Field Strength $\hat{\varepsilon}(\mathrm{V} / \mathrm{cm})$ & $1.00 \times 10^{6}$ & $1.00 \times 10^{6}$ & $1.00 \times 10^{6}$ \\
\hline Rate Constant, $k_{1}^{00}(\mathrm{~cm} / \mathrm{s})$ & $\begin{array}{c}2.306 \times 10^{-} \\
12\end{array}$ & $3.190 \times 10^{-12}$ & $3.422 \times 10^{-12}$ \\
\hline Rate Constant, $k_{3}^{00}\left(\mathrm{~mol}^{-\mathrm{cm}^{2}} \mathrm{~s}\right)$ & $2.895 \times 10^{-18}$ & $3.112 \times 10^{-18}$ & $3.035 \times 10^{-18}$ \\
\hline Rate Constant, $k_{4}^{00}\left(\mathrm{~mol}^{-\mathrm{cm}^{2}} \mathrm{~s}\right)$ & $\begin{array}{c}4.926 \times 10^{-} \\
14\end{array}$ & $3.908 \times 10^{-13}$ & $6.392 \times 10^{-12}$ \\
\hline Rate Constant, $k_{7}^{00}\left(\mathrm{~mol}^{0.4} / \mathrm{cm}^{0.2} \mathrm{~s}\right)$ & $\begin{array}{c}3.000 \times 10^{-} \\
12\end{array}$ & $3.633 \times 10^{-12}$ & $3.604 \times 10^{-12}$ \\
\hline Transfer Coeff.,, & 0.405 & 0.398 & 0.384 \\
\hline Transfer Coeff., & 0.077 & 0.078 & 0.076 \\
\hline Transfer Coeff., & 0.192 & 0.056 & 0.099 \\
\hline
\end{tabular}


Finally, the fact that the rate constant for the passive film dissolution reaction (Reaction 7, Figure II-2.1) and the rate constants for the reactions at the metal/film interface (Reactions 1 and 3, Figure II-2.1) are essentially unaffected by chloride is inconsistent with the chloride-catalyzed film dissolution and chloride penetration models, respectively, for passivity breakdown.

Issues and Concerns: None

\section{III-2. References}

1. G. J. Shugar, T. Ballinger, Chemical Technicians' Ready Reference Handbook, $3^{\text {rd }}$ ed., McGraw Hill, New York, NY, 1990, p. 655

2. E. Sikora and D. D. Macdonald, Electrochim. Acta., 48, 69 (2002).

3. D. D. Macdonald, Pure Appl. Chem., 71, 6, 951 (1999).

4. S. R. Morrison, Electrochemistry at Semicondutor and Oxidized Metal Electrodes, Plenum Press, NY, 1980.

5. G. Barral, F. Njanjo-Eyoki, S. Maximovitch, Electrochim. Acta, 40, 2815 (1995).

6. Y. Okazaki, T. Tateishi, and Y. Ito, Materials Transactions, JIM, 38, 78 (1997).

7. 17 D. D. Macdonald, S. R. Biaggio, and H. Song, J. Electrochem. Soc., 139, 170 (1992).

8. R. P. Frankenthal and J. Kruger (eds.), Passivity of Metals, The Electrochemical Society, Princeton, NJ (1978).

9. M. Urquidi-Macdonald, S. Real, and D. D. Macdonald, Electrochim. Acta, 35(10), 1559 (1990).

10. K-S Lei, D. D. Macdonald, B. G. Pound, and B. E. Wilde, J. Electrochem. Soc., 135, 1625 (1988).

\section{III-3. Task: Crack propagation studies}

\section{$\underline{\text { Task status }}$}

\section{III-3.1 Experimental set up}


The material used in this investigation was a commercial heat of AISI 4340 steel, which was prepared by electric furnace melting and vacuum degassing. The chemical composition is given in Table III-3.1. The as-received steel plate was $38 \mathrm{~mm}$ (1.5 in.) thick. The plate was cut first into blanks and heat-treated according to the procedures given in Table III-3.2. The yield strength was not measured, but the value was estimated to be $\sim 1,490 \mathrm{MPa}$ according to the measured hardness $(48.5 \mathrm{Rc})$ and the typical properties of AISI 4340 steel of this temper. Specimens were machined in the fully heattreated condition. The configuration used for monitoring the coupling current during crack propagation in the compact tension $[\mathrm{C}(\mathrm{T})]$ specimen is shown in Figure III-3.1. The specimen geometry conforms to ASTM E399 based upon $\mathrm{W}=2.997 \mathrm{~cm}(1.18 \mathrm{in}$.). All specimens were prepared in the short transverse-longitudinal (S-L) orientation. Shallow side grooves (5\% of the specimen thickness on both sides) were machined along the crack plane to enhance plane strain and to guide the direction of the growing crack. The effect of reduced thickness $(\mathrm{Bn})$ on the stress intensity was taken into account by replacing $B$ by $\left(B_{n}\right)^{1 / 2}$ in the equation for $K_{I}$, where $B$ is the specimen thickness and $B_{n}$ is the reduced thickness at the groove. The specimens were carefully cleaned and wires were then spot-welded onto the surface. Then, the entire surface of each specimen was coated with baked-on polytetrafluoroethylene (PTFE) for electrical insulation, so that only the crack after fatigue pre-cracking was exposed to the environment. The side cathodes were then mounted and held in place by insulated clips.

TABLE III-3.1

Chemical Composition of AISI 4340 Steel

\begin{tabular}{|c|c|c|c|c|c|c|c|c|c|c|}
\hline Element & $\mathrm{C}$ & $\mathrm{Mn}$ & $\mathrm{P}$ & $\mathrm{S}$ & $\mathrm{Si}$ & $\mathrm{Cu}$ & $\mathrm{Ni}$ & $\mathrm{Cr}$ & $\mathrm{Mo}$ & $\mathrm{V}$ \\
\hline Wt. \% & 0.43 & 0.71 & 0.013 & 0.004 & 0.26 & 0.18 & 1.72 & 0.80 & 0.24 & 0.59 \\
\hline
\end{tabular}

TABLE III-3.2

Heat Treatment and Mechanical Properties of AISI 4340 Steel

\begin{tabular}{|c|ll|}
\hline \multirow{2}{*}{ Heat Treatment } & Normalization $1659 \mathrm{~F}\left(889^{\circ} \mathrm{C}\right)$ & 1 hour Air cool \\
& Austenization $1550 \mathrm{~F}\left(843^{\circ} \mathrm{C}\right)$ & 1 hour Oil quench \\
\hline
\end{tabular}




\begin{tabular}{|c|l|}
\hline & Temper $700 \mathrm{~F}\left(371^{\circ} \mathrm{C}\right) \quad 1$ hour Air cool \\
\hline Mechanical & Hardness, Rc $: 48.5$ \\
Properties & Yield Strength: $1490 \mathrm{MPa}$ (estimated) \\
\hline
\end{tabular}
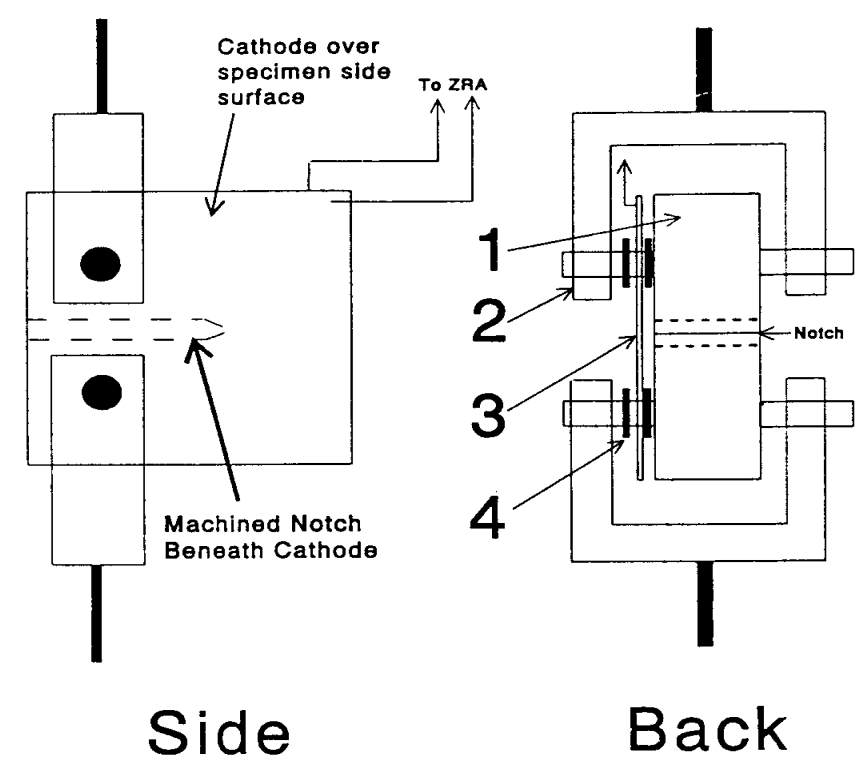

1. Specimen; 2. Clevis; 3. Cathode; 4. PTFE Washer

Figure III-3.1. Specimen configuration used in detecting and measuring the coupling current flowing from a crack to the external metal surface. Note that the $C(T)$ fracture mechanics specimen is coated with PTFE to inhibit the cathodic reduction of oxygen on the specimen surface. Instead, the current flows from the crack to the side cathodes (only one shown) where it is consumed by $\mathrm{O}_{2}$ reduction. The electron current flows from the crack tip to the side cathodes via a zero resistance ammeter, which is used for its measurement.

An MTS 810 universal testing machine was used as the loading apparatus. A special tank made of a nickel-based alloy was used for conducting SCC experiments in hot, concentrated $\mathrm{NaOH}$ solution. Prior to $\mathrm{SCC}$, the $\mathrm{C}(\mathrm{T})$ specimens were pre-cracked to total crack length of $0.40 \mathrm{~W}$ to $0.42 \mathrm{~W}$ as required by ASTM E399 guidelines for load and load shedding at a frequency of $20 \mathrm{~Hz}$. After initiating and propagating the fatigue crack for $\sim 1 \mathrm{~mm}$ under $\mathrm{Kmax}=20 \mathrm{MPa} \vee \mathrm{m}$ with $\mathrm{R}=0.1$, the maximum applied stress intensity was reduced to $<10 \mathrm{ksi} \sqrt{ } \mathrm{in}$. ( $11 \mathrm{MPa} \sqrt{\mathrm{m}}$ ), to yield a sharp crack and to minimize the size of the plastic zone ahead of the crack tip. The load and the load line displacement were monitored at a frequency of $1 \mathrm{~Hz}$. The crack mouth opening displacement (CMOD) 
of the $\mathrm{C}(\mathrm{T})$ specimen was measured using a sensitive linear variable differential transformer (LVDT) and the crack length was evaluated from the CMOD and load, as required by ASTM E399. The validity of using the direct current potential drop (DCPD) method as a suitable technique for crack growth studies under stress corrosion cracking conditions was also explored since it's a very sensitive method for measuring insitu crack lengths during experiments. The position of the current and voltage wires is shown in the below (Figure III-3.2). A similar compact tension specimen was used as a reference sample to account for the change in potential due to factors other than crack growth such as temperature fluctuation and solution concentration fluctuation. A model GPR 1810HD power source was used as the current source and a keithley 2182 nanovoltmeter was used for monitoring voltage.

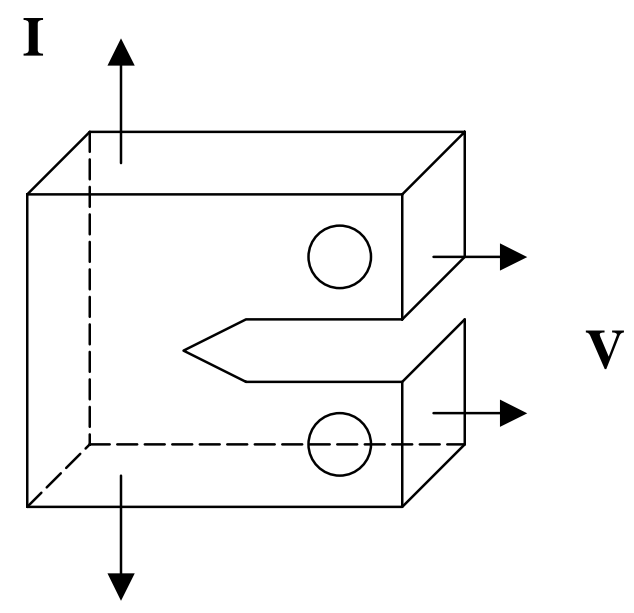

Figure III-3.2. Position of the current and potential wires in the compact tension specimen

The present experiments were carried out at the open-circuit potential in various $(1 \mathrm{M}$ to $12 \mathrm{M}) \mathrm{NaOH}$ solutions, which were prepared from reagent-grade $\mathrm{NaOH}$ and deionized water. The solution was contained in an alkali-resistant vessel fitted with a reflux condenser. The solution was not deaerated and the dissolved oxygen concentration was $\sim 8 \mathrm{ppm}$. Heating was achieved by a heating tape wrapped around the outside of the vessel. The test temperature $\left(70^{\circ} \mathrm{C}\right)$ was measured by a PTFE-coated thermocouple and was controlled to within $\pm 1^{\circ} \mathrm{C}$ using a proportional temperature controller. 
SCC is a typical localized corrosion process that falls within the differential aeration (DA) hypothesis, which requires the spatial separation of the anode and the cathode for crack propagation to proceed. In a DA system with solution of high conductivity, the local anode exists in the region of the system that has the least access to the cathodic depolarizer (i.e., at the crack tip), while the local cathode(s) occurs on those regions that have the greatest access to the depolarizer (external surfaces or at least the entrance region to the crack). Thus, as the crack propagates, the electrons that are released at the crack tip flow through the metal to the external surface, where they are consumed by the reduction of the cathodic depolarizer(s) present in the solution (Figure II-2.1). Accordingly, the internal crack environment is strongly "coupled" to the external surfaces. The magnitude of the current, and hence the crack growth rate, is constrained by the conservation of charge, which is the basis of various "coupled environment" models that have been developed by this group over the past decade to describe corrosion cavity growth [II-2.5]. The (electron) coupling current was monitored using a zero resistance ammeter (ZRA) in the initial tests and using the SI1287 electrochemical interface in its ZRA mode in the later tests. This is shown schematically in Figure III-3.1. Two plate cathodes were mounted on either side of the electrically insulated, pre-cracked $\mathrm{C}(\mathrm{T})$ specimen. Tests were conducted using two sets of cathodes. Initial tests were conducted using AISI 1010 (UNS G10100) carbon steel, which has a similar chemical composition to AISI 4340 steel. The next set of tests was carried out using AISI 1018 carbon steel cathodes. Inserting the ZRA between the sample and the cathodes in this circuit permitted measurement of the coupling current produced during crack extension. Since the distance over which positive current can travel as it exits the crack mouth is limited by the conductivity of solution, the cathodes were placed on the sides of the $C(T)$ specimen in close proximity to the intersection of the crack plane with the surface. The potential of the specimen was also measured with respect to a platinum electrode using a high impedance voltmeter in the initial tests and with respect to $\mathrm{Ag} / \mathrm{AgCl}$ reference electrode by using the SI1287 electrochemical interface in the later tests. The platinum reference electrode was placed $2 \mathrm{~mm}$ from the crack tip on one side of the specimen in the earlier tests, but in the later tests the $\mathrm{Ag} / \mathrm{AgCl}$ electrode was placed away from the crack tip and at a distance of $2 \mathrm{~mm}$ from the cathodic surface in an attempt to measure the noise in the 
mixed potential rather than the noise at the crack tip potential. The noise data was acquired at a frequency of $10 \mathrm{~Hz}$. A fast Fourier transform (FFT) algorithm was used to transform data from the time domain into the frequency domain, in order that periodic components in the (electron) coupling current could be detected and identified. After each fracture experiment, the fracture surfaces were examined by scanning electron microscopy (SEM).

AESmart 2000 acoustic emission apparatus fitted with a SE9125-M data transducer was used for monitoring the acoustic emission signals during crack growth. An HF/LF ratio of 1 was used. The system was configured for single channel use. The AESmart system eliminates extraneous noise sources before they enter the database, and for the first time, for any $\mathrm{AE}$ instrumentation, give information regarding the depth of a growing crack in a plate.

\section{III-3.2 Identification of the Ideal Parameters to Describe Crack Propagation}

One of the main aims of the current work was to identify the individual microfracture events in order to study their repassivation behavior, frequency of occurrence, etc., during the caustic cracking of AISI 4340 steel. Hence, it was necessary to identify the ideal conditions under which the crack propagates via individual microfracture events that can be temporally resolved.

Tests were conducted at $70^{\circ} \mathrm{C}$ at different solution concentrations (starting from $12 \mathrm{M} \mathrm{NaOH}$ down till $1 \mathrm{M} \mathrm{NaOH}$ ) and stress intensity values. Though cracking was observed giving rise to characteristic current and potential noise signals, temporal resolution of the individual microfracture events could only be realized at a solution concentration of $6 \mathrm{M} \mathrm{NaOH}$ and at an initial stress intensity factor of $27.4 \mathrm{MPa} \sqrt{m}$. No crack growth was observed at lower solution concentrations. The crack growth rates and the time for failure at different values of $\mathrm{NaOH}$ concentrations and initial stress intensity factor are given in Table III-3.1. Data at higher concentrations were analyzed in the frequency domain. 
TABLE III-3.1

Crack growth rate under various conditions

\begin{tabular}{|c|c|c|c|}
\hline $\begin{array}{c}\text { Solution } \\
\text { concentration } \\
(\mathrm{M})\end{array}$ & $\begin{array}{c}\text { Initial stress } \\
\text { intensity } \\
\text { factor }(\mathrm{MPa} \sqrt{\mathrm{m}})\end{array}$ & $\begin{array}{c}\text { Crack growth } \\
\text { rate }\left(1^{\text {st }} \text { stage }+\right. \\
\left.2^{\text {nd }} \text { stage }\right)\left(10^{-8}\right. \\
\mathrm{cm} / \mathrm{s})\end{array}$ & $\begin{array}{c}\text { Micro fracture } \\
\text { event dimension } \\
(\mu \mathrm{m})\end{array}$ \\
\hline 12 & 13 & 4700 & $\begin{array}{c}\text { Cannot be resolved } \\
\text { in time domain }\end{array}$ \\
\hline 8 & 25 & 1000 & $\begin{array}{c}\text { Cannot be resolved } \\
\text { in time domain }\end{array}$ \\
\hline 6 & 27.4 & 5.3 & No crack \\
\hline 5 & 14 & 0 & propagation \\
\hline
\end{tabular}

\section{III-3.3 Frequency Domain Analysis for High Solution Concentrations}

Tests were initially conducted at higher solution concentrations starting from 12M. The potential and the (electron) coupling current were recorded over the time period from 10 minutes before load application to final fracture, which was due to mechanical overload, as shown in Figure III-3.3. Small random fluctuations in the (electron) coupling current have been shown to be characteristic of uniform corrosion, and indeed this was the pattern that was observed before the load was applied to the specimen [Figures III-3.3(b) and III-3.3(a)]. Prior to loading, the (electron) coupling current was found to have a positive sign, corresponding to the flow of electrons through the metal from the cathodes to the specimen. This is most likely due to a small galvanic effect arising from the fact that the cathodes and the specimen are fabricated from slightly different materials. However, upon loading, the (electron) coupling current that flows between the specimen and the cathode first decreased to a lower value and eventually 
became negative, corresponding to electron flow from the specimen to the cathodes (or positive ion flow from the crack through the solution to the cathode surfaces) [Figure III3.3(b)]. Thus, during the first stage of the experiment (first 70 minutes after loading), the crack propagates at a very low growth rate, which is dictated by the existence of a residual compressive stress zone ahead of the tip of the fatigue precrack. During this period, the (electron) coupling current changes from positive to negative and the amplitude of the current fluctuations increases gradually with increasing crack length [Figures III-3.3(b) and III-3.4(b)]. During the second stage of the experiment (from 70 to 142 minutes), the crack growth rate is about $8.3 \times 10^{-5} \mathrm{~cm} / \mathrm{s}(3 \mathrm{~mm} /$ hour $)$, which is significantly larger than that observed during the first stage $\left(1.7 \times 10^{-5} \mathrm{~cm} / \mathrm{s}\right)$. The (electron) coupling current and the fluctuation amplitude $(\sim 10 \mu \mathrm{A})$ remain almost constant, as shown in Figures III-3.4(c) and (d). During the final stage of fracture (after 142 minutes), the crack propagates rapidly, ultimately resulting in complete fracture by mechanical overload. The amplitude of the electron current noise increases to $20 \mu \mathrm{A}$ during the final stage [Figure III-3.4(d)], which is twice that observed during the second stage of the experiment.
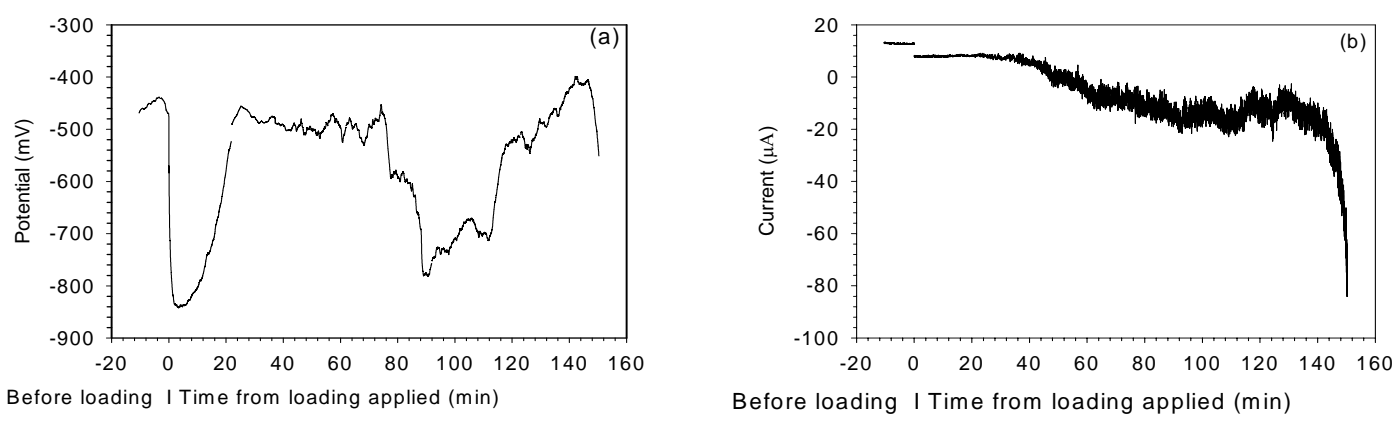

Figure III-3.3. Specimen potential (a) and (electron) coupling current (b) versus time for fracture in AISI 4340 steel in $12 \mathrm{M}$ sodium hydroxide solution at $70^{\circ} \mathrm{C}$.

The observations summarized above confirm, in a qualitative manner, that the magnitude of the (electron) coupling current reflects the rate of crack propagation, a finding that is in concert with that of our previous work on crack propagation in sensitized Type $304 \mathrm{SS}$ in high temperature aqueous solutions [II-2.4]. The new 
observation from the present work is that the amplitude of the noise may also be related to the crack propagation rate.

The potential is found to shift in the negative direction promptly upon application of the load, but then recovers gradually as the load is maintained [Figure III-3.3(a)]. This transition is probably related to the opening of the fatigue pre-crack, such that the freshly fractured surfaces of the fatigue precrack are exposed to the solution. The potential of the specimen then shifts in the negative direction as the growth rate increases at the beginning of the second stage, and then recovers (shifts in the positive direction) due to repassivation as the crack is opened towards final fracture due to the ever increasing stress intensity. During the final stage, both the (electron) coupling current and the potential shift sharply in the negative direction simultaneously (Figure III-3.3), because of the large fresh surface that is exposed to the solution by fast fracture due to mechanical overload. It is postulated that the potential measured here is actually the mixed potential between the CT specimen and the cathodes, rather than the potential at the (emergent) crack tip. This is because the reference electrode was located near the emergent crack tip on one side of specimen, but was also near a cathode that was connected to the specimen through the ZRA.The data reported here clearly demonstrate that the positive current flows through the solution from the crack tip to the external cathode during crack propagation in AISI 4340 steel in concentrated sodium hydroxide solution at $70{ }^{\circ} \mathrm{C}$. Accordingly, these data confirm that the cathodic reaction is not restricted to the flanks of the crack or to the crack mouth, but that it also occurs on the external surfaces, as postulated in the CEFM model [II-2.15]. The magnitude of the (electron) coupling current and the amplitude of the current noise increase with increasing crack growth rate. Finally, periodic pulses in the (electron) coupling current recorded during crack propagation show that caustic-cracking advances by discrete microscopic fracture events, as discussed below. Figure III-3.5 shows an amplitude spectrum that was obtained by transforming the (electron) coupling current from the time domain into the frequency domain by using the Fast Fourier Transform (FFT). The results indicate that the event spacing in frequency space increases while, simultaneously, the amplitude decreases with increasing frequency. This is consistent with crack advance via many small micro fracture events, characterized by small current amplitudes, occurring more-or-less 
simultaneously, superimposed upon less frequent, but larger discrete events of higher current amplitude. The latter events apparently occur at frequencies between 0.02 and $0.1 \mathrm{~Hz}$, as shown in Figures III-3.5 (b) and (c). The apparent transients at frequencies below $0.02 \mathrm{~Hz}$ are considered to arise from DC drift and hence may be artifacts. Before the application of the load, there are no micro fracture events, and the corresponding (electron) current amplitudes at various frequencies are very small (Figure III-3.5 (a)) and are characteristic of general corrosion.
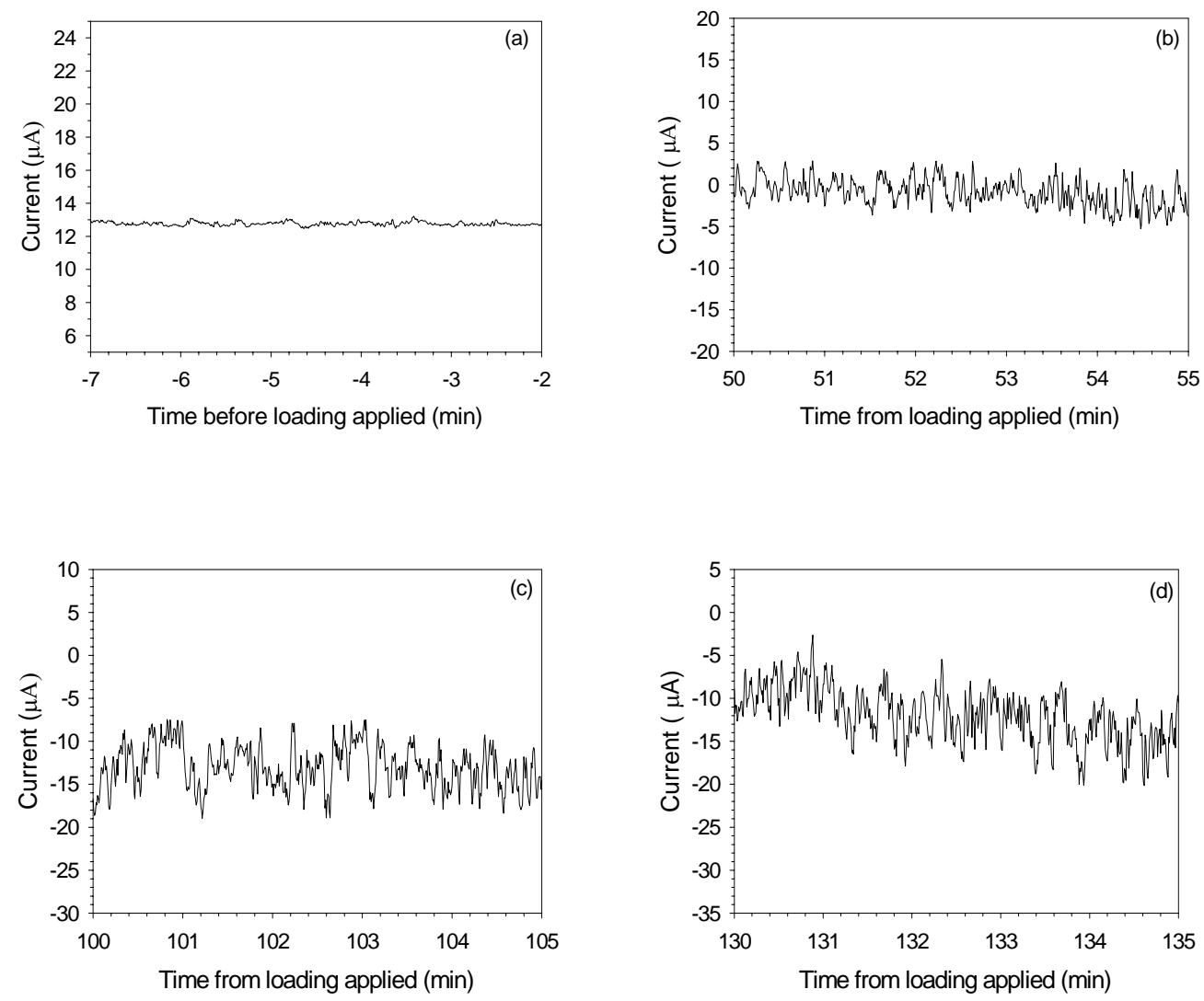


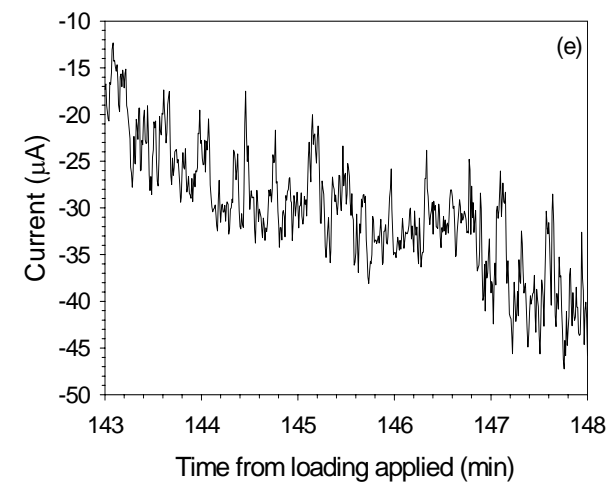

Figure III-3.4. Details of the electron current noise at various stages in the fracture history. Before loading (a) and after loading application (b, c, d and e) in $12 \mathrm{M}$ sodium hydroxide solution at $70{ }^{\circ} \mathrm{C}$.

Examination of the fracture surface by optical microscopy shows brittle morphological characteristics. The stress corrosion crack remains in the original precrack plane with no tendency for macro crack branching. Fractographic studies of the cleaned surface show that the crack propagation path in caustic solution is typical of intergranular fracture [right part in Figure III-3.6 (a)] following the transition zone, which is characterized by mixed intergranular/transgranular morphology near the front of the fatigue pre-crack [left part in Figure III-3.6 (a)]. The final fracture zone is predominantly dimpled rupture [Figure III-3.6 (b)], which is characteristic of mechanical overload.
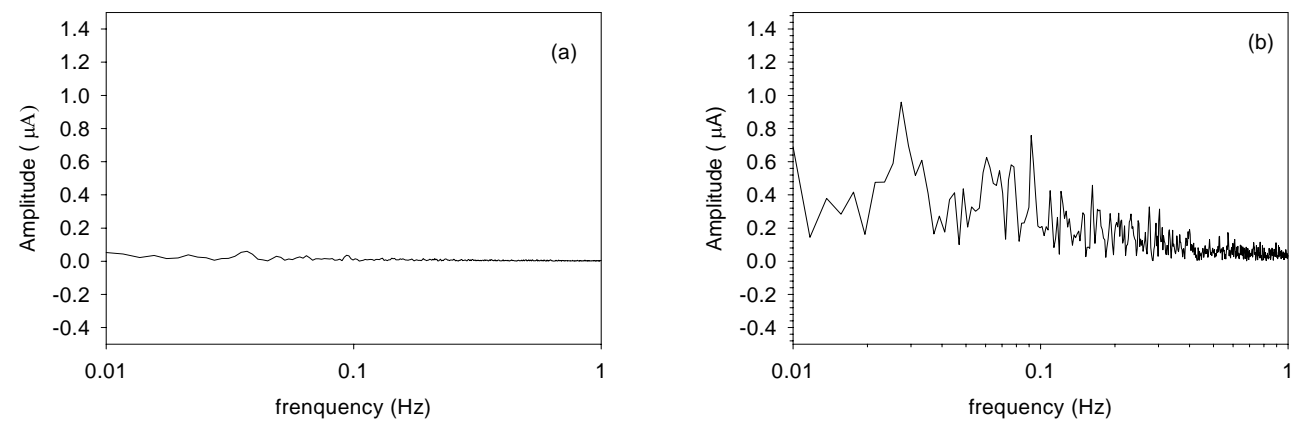


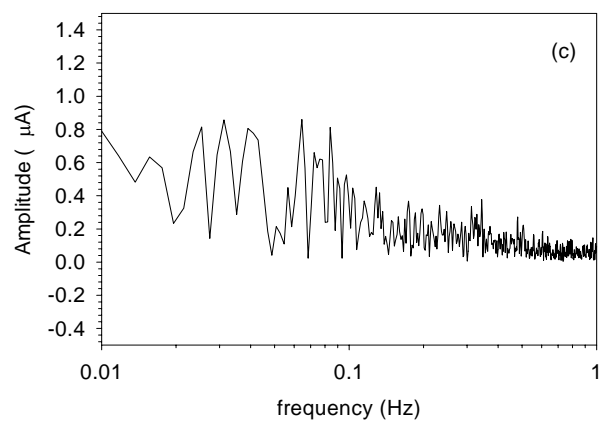

Figure III-3.5. Amplitude vs. frequency after FFT transformation of the (electron) coupling current for AISI 4340 steel in $12 \mathrm{M} \mathrm{NaOH}$ solution at $70{ }^{\circ} \mathrm{C}$. (a) Before loading application; (b) during the second stage of fracture; and (c) before final fracture of the specimen.



(a)

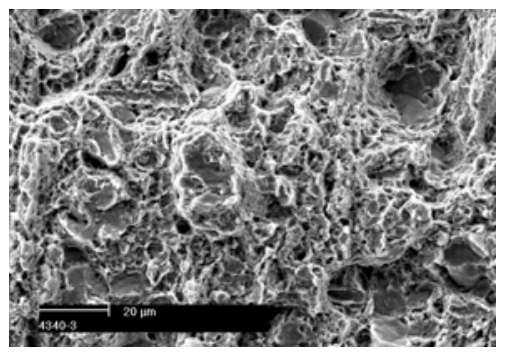

(b)

Figure III-3.6. SEM fractographs of SCC fracture surfaces produced at the free corrosion potential in $12 \mathrm{M}$ $\mathrm{NaOH}$ solution at $70{ }^{\circ} \mathrm{C}$. (a) Crack propagating from the fatigue pre-crack front (left) in mixed form to caustic cracking (right) in intergranular form. (b) The final fracture zone showing predominantly dimpled rupture (mechanical overload).

Assuming that the frequency of the brittle micro fracture events at the crack tip decreases as the hydroxide concentration is lowered, thereby resulting in a lower crack growth rate, it must be possible to select conditions such that the fracture events are separated temporally and hence can be examined individually. Hence tests were carried out at successively lower $\mathrm{NaOH}$ concentrations. The results were similar to the results obtained at $12 \mathrm{M} \mathrm{NaOH}$ though the crack growth rate decreased with decreasing solution concentration. Individual microfracture events could be resolved only at a concentration 
of about $6 \mathrm{M} \mathrm{NaOH}$. The data obtained under these conditions are discussed in the next part.

\section{III-3.4 Time domain analysis for Lower Solution Concentrations - 6 M NaOH}

In this experiment, the specimen was loaded to $998 \mathrm{~kg}$ (2200 pounds) corresponding to an initial stress intensity factor of $27.4 \mathrm{MPa} \sqrt{m}(25 \mathrm{ksi} \sqrt{\text { in }})$. Low amplitude, random fluctuations in the (electron) coupling current were observed prior to loading, as shown in Figure III-3.7, which are characteristic of uniform corrosion as stated previously. The (electron) coupling current is displaced in the negative direction promptly upon load application, but then recovers gradually to almost the same level, and has the same fluctuation characteristics, as that observed before loading. This transition is attributed to the exposure and subsequent passivation of the surfaces of the fatigue precrack to the solution upon the initial application of the load. The noise in the (electron) coupling current recorded during the subsequent 3 hours period from loading shows no obvious change, except for a small DC drift.

Four hours later, a significant transient was observed in the (electron) coupling current. Typical data collected after 4 hours from load application are shown in Figure III-3.8. In general, the (electron) coupling current contains individual, low frequency transients with amplitudes ranging from 1.5 to $7 \mu \mathrm{A}$. A typical pattern in the current noise is shown in Figure III-3.9. The transient in the (electron) coupling current is again characterized by a rapid drop and slow recovery. This type of noise in the (electron) coupling current appears to be quite characteristic of caustic SCC crack propagation.

The kinetics of repassivation of brittle micro fracture events is an issue of considerable theoretical importance in formulating models for crack propagation. The kinetics for the case shown in Figure III-3.9 are explored in Figure III-3.10, where a first order plot of the relaxation in the coupling current is attempted. The current is indeed found to decay in a first order fashion with a rate constant of $0.029 \mathrm{~s}^{-1}$. 


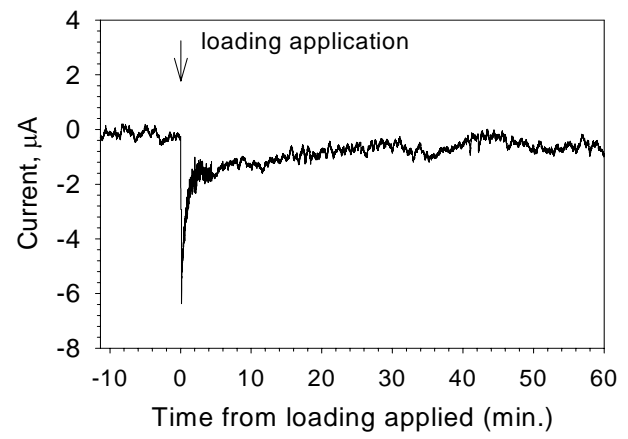

Figure III-3.7. The coupling current vs. time before and after load application in $6 \mathrm{M} \mathrm{NaOH}$ at $70{ }^{\circ} \mathrm{C}$.

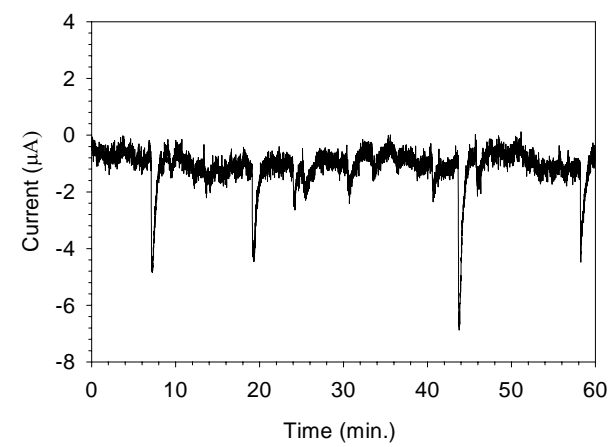

Figure III-3.8. Typical transients in the (electron) coupling current from 5 to 52 hours after load application for AISI 4340 steel in $6 \mathrm{M} \mathrm{NaOH}$ solution at $70{ }^{\circ} \mathrm{C}$.

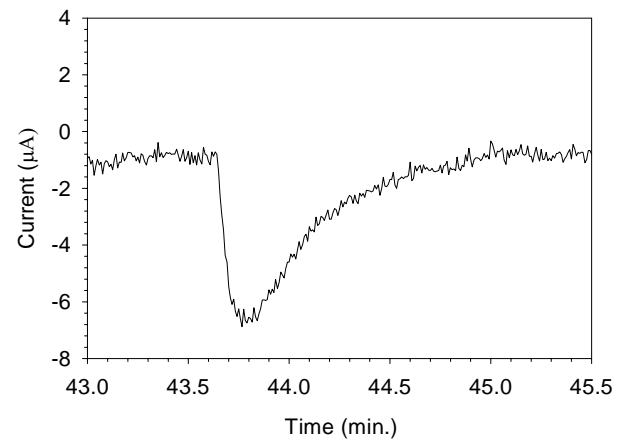

Figure III-3.9. Typical transient in the (electron) coupling current for a single fracture event in AISI 4340 steel in $6 \mathrm{M} \mathrm{NaOH}$ solution at $70{ }^{\circ} \mathrm{C}$. 




Figure III-3.10. First order kinetic plot of the repassivation current shown in Figure III-3.9

The crack propagated for about $100 \mu \mathrm{m}$ in length in intergranular form (observed by SEM) within the 52-hour observation period after loading. The average crack growth rate was $5.3 \times 10^{-8} \mathrm{~cm} / \mathrm{s}$. The results show that temporally resolved brittle micro fracture events can be observed during caustic SCC, provided that the crack growth rate is sufficiently low. Previous work [II-2.4] on the intergranular stress corrosion cracking of Type $304 \mathrm{SS}$ in high temperature aqueous systems showed that the pulses in the coupling current were of a single frequency (or at least of a very narrow band in frequency) and that the fluctuations occurred in "packets" of four to thirteen fluctuations separated by brief periods of high frequency noise. Interestingly, the crack growth rate in this system was about $3 \times 10^{-7} \mathrm{~cm} / \mathrm{s}$, or about seven times higher than that observed here. In both cases, the quantity CGR/f, where CGR is the crack growth rate and $\mathrm{f}$ is the frequency of transients, is similar $\left(\sim 1.5\right.$ to $\left.5 \times 10^{-7} \mathrm{~cm}\right)$, suggesting that the micro fracture dimensions are also similar. The noise signature indicates that the crack advances by one brittle micro fracture event at a time [II-2.4], probably occurring on a less-than-favorably oriented grain face with respect to the principal stress vector. Once the crack traverses the grain face and intersects a favorably oriented face (note that the crack propagates intergranularly), the crack advances rapidly, resulting in the burst of high frequency, low amplitude noise.

For AISI 4340 steel in $\mathrm{NaOH}$ solution at $70{ }^{\circ} \mathrm{C}$, the results show that the susceptibility to caustic SCC increases with $\mathrm{NaOH}$ concentration. No crack propagation is observed in $1 \mathrm{M}$ and $5 \mathrm{M} \mathrm{NaOH}$ solutions, and the noise in the (electron) coupling 
current comprises low amplitude, random fluctuations that are characteristic of general corrosion. Individual brittle micro fracture events are observed at low crack growth rate in $6 \mathrm{M} \mathrm{NaOH}$ solution. In more concentrated $\mathrm{NaOH}$ solution $(8 \mathrm{M}$ and $12 \mathrm{M}$ ), on the other hand, the noise in the (electron) coupling current displays periodic features that are best observed by Fourier transformation. It is postulated that caustic cracking in this high strength steel in the more concentrated caustic environments advances by many micro cracks occurring simultaneously. A transient in the coupling current signals each micro crack advance and, since many events occur at any given time, the coupling current appears as semi random fluctuations on a high mean.

Hydrogen embrittlement has been postulated to be the mechanism for environment-assisted cracking in high strength steels in caustic environments [1]. Substantial evidence exists in the literature that the chemical environment at the tip of a stress corrosion crack can differ markedly from the bulk solution composition, due to the "occluded cell" (differential aeration) effect. Brown et al. [2, 3] found that the solution at the tip of a crack in AISI 4340 steel in chloride containing solution always had a $\mathrm{pH}$ of 3.5 to 3.9 , even though the bulk pH varied between 2 and 10. The establishment, in that case, of a steady state $\mathrm{pH}$ at the tip is due to hydrolysis equilibria involving the soluble corrosion products (e.g., $\mathrm{Fe}^{2+}$ ).

The potential at the crack tip for high-strength steel always lies at or below the "hydrogen line" for the prevailing crack tip $\mathrm{pH}$, for a variety of external conditions. Thus, the observations of this work can be explained by a brittle micro fracture mechanism, in which the events are possibly induced by hydrogen. We postulate that the large current exiting the crack mouth, which has been detected by the ZRA measurements reported here, generates a lower $\mathrm{pH}$ at the crack tip and that hydrogen is injected into the local metal matrix ahead of the crack tip. Hydrogen atoms then diffuse to the grain boundaries where embrittlement occurs by pressurization of micro voids or by chemical decohesion via reaction with segregated metalloids (e.g., P, Si, N) [4]. In any event, the periodic fracture events are initiated at points ahead of the crack at which the hydrostatic stress and the hydrogen concentration exceed critical conditions. This mechanism is proposed, in spite of the fact that the environment external to the crack is highly alkaline and oxidizing. Furthermore, we note that the fractographic characteristics of caustic cracking 
in AISI 4340 steel in $\mathrm{NaOH}$ solution, as observed in this work, are very similar as those found for hydrogen embrittlement in precracked specimens of the same steel in gaseous hydrogen [5], an environment in which anodic dissolution is not possible.

Finally, assuming that the fracture events are semi-circular in geometry of radius $r$ and that the frequency at which they occur is $f$, the crack growth rate can be written as

$$
\frac{d L}{d t}=\frac{2 r^{2} f}{B_{n}}
$$

where $B_{n}$ is the specimen thickness at the groove. Equation (1) is readily rearranged to yield the micro fracture dimension as

$$
r=\sqrt{\frac{B_{n} \cdot d L / d t}{2 f}}
$$

For the $6 \mathrm{M} \mathrm{NaOH}$ case, $d L / d t=5.3 \times 10^{-8} \mathrm{~cm} / \mathrm{s}, B_{n}=2.7 \mathrm{~cm}$, and $f \approx 0.003 \mathrm{~s}^{-1}$ (Figure III-3.8), yielding a micro fracture dimension of $\approx 49 \mu \mathrm{m}$. Note that the fracture frequency is somewhat subjective, because it depends upon what is counted as a "fracture event". In the present case, eleven events were counted over the sixty minutes recorded in Figure III-3.8, resulting in the value given above for $f$. Acoustic emission test results in the second heat of samples confirm the assumption that the individual transients indeed represent individual crack events in most of the cases. The calculated fracture dimension may be compared with the $3 \mu \mathrm{m}$ dimension found for the fracture of sensitized Type 304 $\mathrm{SS}$ in high temperature water [II-2.2]. It is likely, however, that larger micro fracture events give rise to the more intense current transients, so that the $49 \mu \mathrm{m}$ dimension estimated above probably represents the upper end of a distribution in this quantity. Because the dimension of a slip event should be a small multiple of the Burger's vector (nanometers), the fracture dimension appears to be much too large to be consistent with the slip/dissolution/repassivation mechanism for crack advance. On the other hand, the dimension is consistent with a hydrogen embrittlement mechanism, noting that the fracture dimension is expected to correspond with the spacing of some metallurgical 
asperity (e.g., precipitates) on the grain boundary or with the grain size itself (note that the grain size as revealed on the fracture surface is $10-40 \mu \mathrm{m}$, Figure III-3.6).

\section{Issues and concerns : Tests with AISI 1018 steel cathodes}

Tests were conducted under the same conditions, on the same material using AISI 1018 carbon steel cathodes. In this case crack extension was monitored simultaneously using AET. Though the tests conducted under these conditions yielded similar transients, some striking differences were noted. These differences are summarized below and discussed in subsequently.

1. Crack growth rate depends significantly on the external cathode

2. Kinetics of repassivation also changes significantly with the external cathode

3. Crack growth rate is a sensitive function of the distance of separation between the external cathode and the crack tip - Crack growth stops at higher distances of separation

These results have important implications on the crack growth rate mechanisms which will be discussed later in the section. The experimental results for these tests are given below.

Similar low amplitude, random fluctuations in the (electron) coupling current were observed prior to loading, as shown in Figure III-3.11. The (electron) coupling current is displaced in the negative direction promptly upon load application, but then recovers gradually, and has similar fluctuation characteristics, as that observed before loading. The noise in the (electron) coupling current recorded during the subsequent 4 hours period from loading shows no obvious change, except for a small DC drift. There was no crack growth in the first four hours which has been confirmed from the acoustic emission data (Figure III-3.12). The first transient which appears at the end of four hours coincides with the first significant spike in the acoustic emission counts. An hour of data acquired after four hours of load application is shown in Figure III-3.13. 


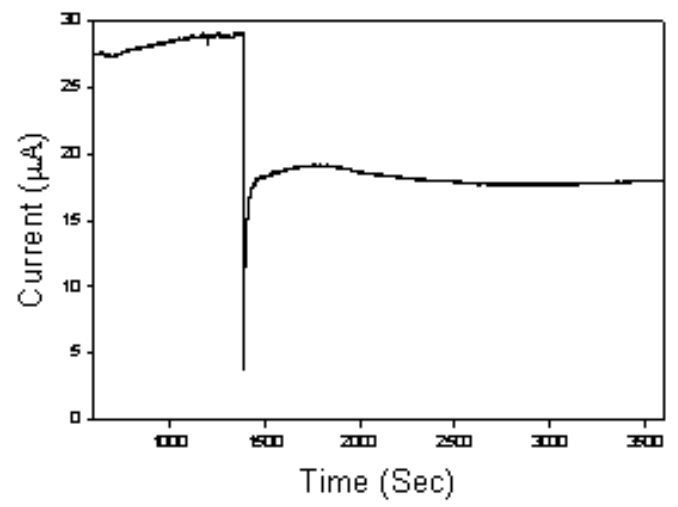

Figure III-3.11. The (electron) coupling current vs. time before and after load application in $6 \mathrm{M} \mathrm{NaOH}$ at $70{ }^{\circ} \mathrm{C}$ for the second heat sample.

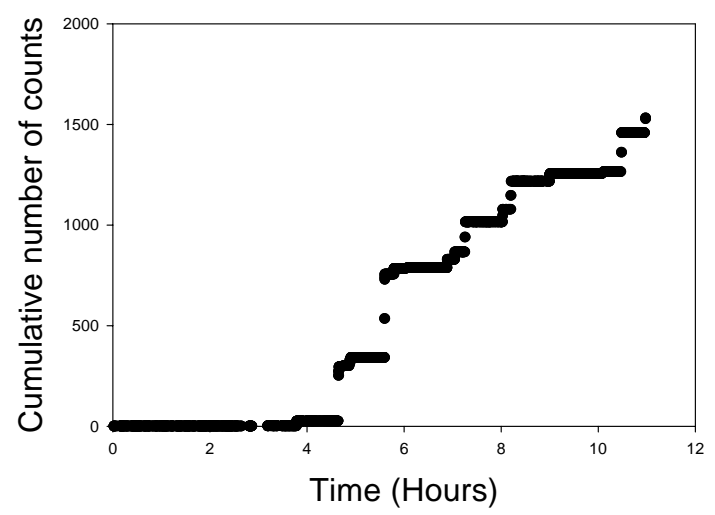

Figure III-3.12. Cumulative acoustic emission counts vs. time after the application of load for the second heat sample

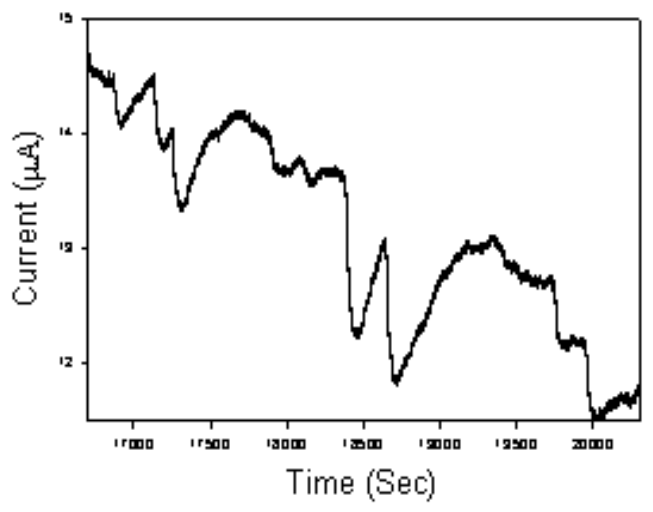


Figure III-3.13. Typical transients in the (electron) coupling current 4 hours after load application for AISI 4340 steel in $6 \mathrm{M} \mathrm{NaOH}$ solution at $70{ }^{\circ} \mathrm{C}$ for the second heat sample

Notice that these samples take significantly longer times to repassivate in comparison to the previous tests where AISI 1010 cathodes were used (Figure III-3.13). We can also notice that the repassivation time is more than the time of separation between two crack events in most of the cases. This leads to accumulation of coupling current. Only very few crack events that were observed over a period of six hours underwent complete repassivation. This was not the case when AISI 1010 cathodes were used (Figure III-3.8).

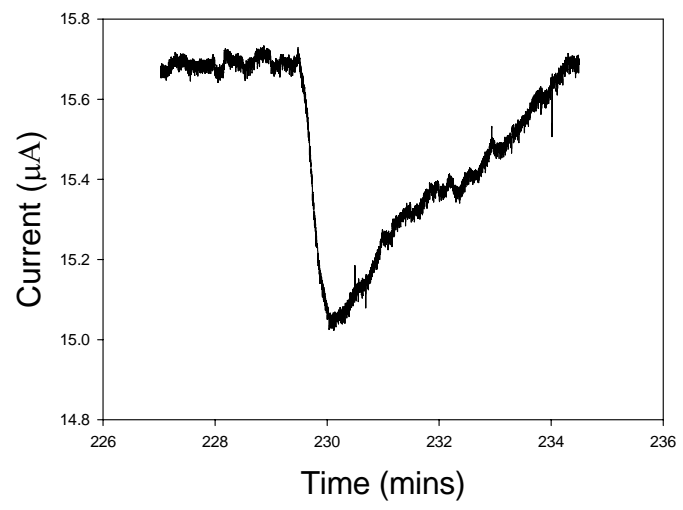

Figure III-3.14. Typical transient in the (electron) coupling current for a single fracture event in AISI 4340 steel in $6 \mathrm{M} \mathrm{NaOH}$ solution at $70{ }^{\circ} \mathrm{C}$ for the second heat sample

The current in this case is still found to decay in a first order fashion, as before, but at a much slower rate as indicated by the rate constant of $0.00026 \mathrm{~s}^{-1}$ (Figure III-3.15). Recall that the rate constant in the previous case was calculated to be $0.026 \mathrm{~s}^{-1}$, which is more than an order of magnitude larger than this. 


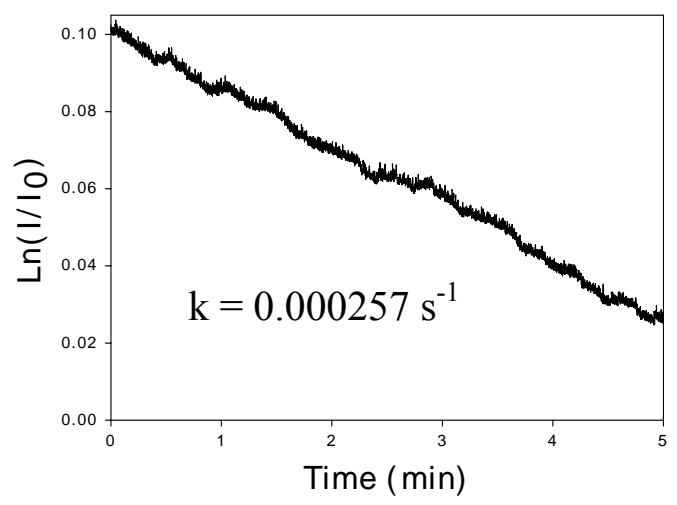

Figure III-3.15. First order kinetic plot of the repassivation current shown in Figure III-3.14

The acoustic emission event rate is commensurate with the current noise event frequency. For example, the first transient in the coupling current (Figure III-3.14) which occurs roughly 230 minutes after the application of load exactly coincides with the first significant increase in the AE counts (Figure III-3.16). In the subsequent 6 hours of data acquisition, the coupling current transients coincided with the $\mathrm{AE}$ counts in most cases. This indicates that the individual transients in the coupling current are indeed due to the individual microfracture events as postulated.

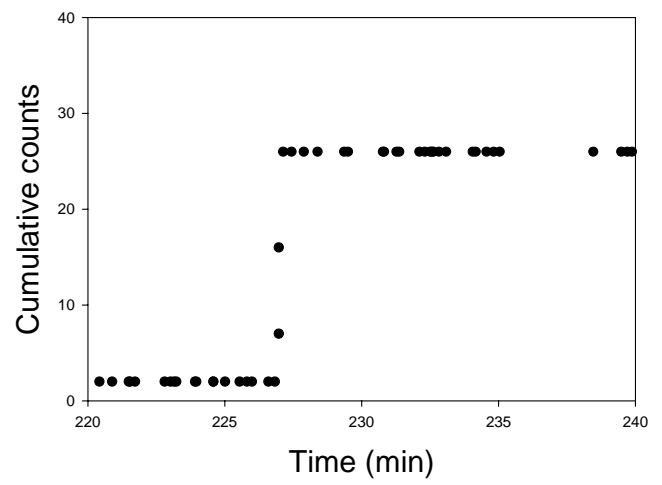

Figure III-3.16. First spike in the acoustic emission count corresponding to the first crack event

The results of these tests have important implications which are discussed below.

- The fact that the crack growth rate decreased significantly in the second case (using AISI 1018 cathodes) implies that the crack growth rate is a sensitive function of the external cathodic environment. 
- It also signifies that most of the cathodic reaction takes place in the external cathode and not the crack flanks which is evident from the fact that the crack propagation stops completely at higher distances of separation between the external cathode and the crack tip.

- These results finally confirm the existence of a strong coupling between the crack tip and the external environment which is proposed by the coupled environment fracture model.

To get better understanding of the mechanisms in a quantitative sense we propose to do the following tasks in the near future.

1. To enhance EES as a suitable corrosion monitoring technique

2. To develop a chemical potentiostat using which the potential at the crack tip can be varied in order to study its effect on the crack growth rate.

3. To study the effect of temperature, concentration of the solution and other factors on the crack growth rate and repassivation kinetics in order to develop a comprehensive model that could help to deterministically predict the life of containers used for storing high level nuclear wastes.

4. To quantitatively study the effect of the distance of separation between the external cathode and the crack tip on the crack growth rate.

5. To quantitatively study the effect of enhancing the kinetics of the cathodic reaction on the external cathode surface (by platinizing) on the crack growth rate.

\section{References III-3:}

17. R. C. Newman and R. P. M. Procter, British Corrosion Journal, 25, 259 (1990).

18. B. F. Brown, ed., Stress Corrosion Cracking in High Strength Steels and in Titanium and Aluminum Alloys, Naval Research Laboratory, 1972, p.80-145

19. B. F. Brown, Stress Corrosion Cracking and Hydrogen Embrittlement of Iron Base Alloys, Unieux-Firminy, France, June 12-16, 1973, NACE International, USA, p.747-750

20. B. Craig, ASM Handbook, ASM International, 13, Corrosion, 163 (1990)

21. D. Hardie and S. Liu, Corros. Sci., 38, 721 (1996). 


\section{III-4 Task: Electrochemical Emission Spectroscopy}

This task consists of three parts: experimental replication of EES noise data, computer simulation of same, and analysis of the noise data for key parameters relating to types of corrosion. The first part is to model the electrochemical environment of nuclear waste storage containers in order to replicate the EC noise data for general and localized corrosion of the actual containers. Secondly, a computer model of the anodic sites, the cathodic sites, and their interaction, on the corroding metal surfaces, is generated. From this model the current and voltage noise data is derived, and the model is improved until the simulated data compares with the experimental data. This model gives a greater understanding of the physical arrangement of the metal surface, and of the electrochemistry of the reaction sites, and it depends on the experimental data for a basis of comparison. The simulated data, the general and localized corrosion from this task, and the general corrosion and SCC data from the other tasks are analyzed using Wavelet Analysis to provide a definitive means to differentiate between types of corrosion.

\section{$\underline{\text { Task Status: }}$}

Currently an electrochemical cell is being constructed for the collection of EC noise from general and localized corrosion. The cell is a four electrode arrangement with two identical type 4340 steel specimen electrodes, a standard reference electrode $(\mathrm{Ag} / \mathrm{AgCl}$ electrode with saturated $\mathrm{KCl}$ solution), and a pseudo-reference electrode (Pt wire electrode). The cell will be maintained at a temperature of between 90 and 100 degrees centigrade, the approximate temperature conditions inside the storage containers. The open circuit potential and current is measured simultaneously while the sample electrodes undergo corrosion. To initiate corrosion, the steel specimens are immersed in a saturated hydroxide solution. To further mimic the conditions inside the waste storage containers and to generate localized corrosion, four chemical solutions are added to the hydroxide solution in varying permutations. The four chemicals used are $\mathrm{NaCl}$ (sodium chloride), $\mathrm{NaNO}_{2}$ (sodium nitrite), $\mathrm{NaPO}_{3}$ (sodium nitrate), and $\mathrm{NaHPO}_{4}$. These four chemicals contain aggressive ions known to encourage corrosion, and in the various 
combinations they effectively simulate the chemical environment of any given storage container. These chemicals are introduced in varying combinations and permutations to better understand the effects that each has on the EC noise signal, and how they effect (impede or catalyze) each other.

Issues and Concerns: $\quad$ None

\section{III-5. Task: Combining the Deterministic and Statistical Approaches for Predicting Localized Corrosion Damage}

\section{III-5.1. Developing a Theoretical Basis for DFA}

\section{$\underline{\text { Task Status }}$}

Localized corrosion damage in an arbitrary system is completely defined if we know how many pits or other corrosion events $\left(\right.$ per $\mathrm{cm}^{2}$ ) have depths between $x_{1}$ and $x_{2}$ $\left(x_{1}<x_{2}\right)$ for a given observation time, $t$. Let us denote this number by $\Delta N_{k}\left(x_{1}, x_{2}, t\right)$. Here, index $\mathrm{k}$ denotes different types of corrosion event (e.g., pits or cracks). Instead of employing a function with three variables, $\Delta N_{k}$, it is more convenient to use a function of two variables - the integral damage function (IDF), $F_{k}(x, t)$. This function is defined as the number $\left(\right.$ per $\mathrm{cm}^{2}$ ) of corrosion events with depths larger than $\mathrm{x}$ for a given observation time, $t$. It is evident that

$$
\Delta N_{k}\left(x_{1}, x_{2}, t\right)=F_{k}\left(x_{1}, t\right)-F_{k}\left(x_{2}, t\right)
$$

In turn, it is convenient to express the integral DF, $F_{k}(x, t)$, via the differential DF, $f_{k}(x, t)$, using the relation

$$
F_{k}(x, t)=\int_{x}^{\infty} f_{k}\left(x^{\prime}, t\right) d x^{\prime} \quad \text { or } \quad f_{k}(x, t)=-\frac{\partial F_{k}(x, t)}{\partial x}
$$

The differential DF for defects of type $k, f_{k}$, is defined from the condition that $f_{k}(x, t) d x$ is the number of defects $k$ (per $\mathrm{cm}^{2}$ ) having a depth between $x$ and $x+d x$ for a given 
observation time, $t$. It is evident that the set of functions $f_{k}$ for all types of defects yields complete information about damage in the system. The advantage of using the differential DF lies in the fact that it obeys a simple differential equation (see below) and accordingly can be calculated for any given set of conditions. Of course, all of the functions $\Delta N_{k}\left(x_{1}, x_{2}, t\right), F_{k}(x, t)$, and $f_{k}(x, t)$ may depend, in the general case, on position on the metal surface, but in this analysis we will assume that all sites belong to a given ethnic population.

The function $f_{k}$ has dimension of $\# /\left(\mathrm{cm}^{2} \mathrm{~cm}\right)=\# / \mathrm{cm}^{3}$ (analogous to the concentration of a particle). Accordingly, it is very convenient to regard each defect as a "particle" that moves in the $\mathrm{x}$ direction (perpendicular to the surface, with $\mathrm{x}=0$ being at the metal surface). The coordinate of this particle, $x$, coincides with the depth of penetration of the defect. Accordingly, $f_{k}$ can be regarded as the concentration of particles and hence must obey the law of mass conservation,

$$
\frac{\partial \mathrm{f}_{\mathrm{k}}}{\partial \mathrm{t}}+\frac{\partial \mathrm{j}_{\mathrm{k}}}{\partial \mathrm{x}}=\mathrm{R}_{\mathrm{k}}, \quad k=1,2, \ldots, K
$$

where $j_{k}$ and $R_{k}$ are the flux density and the bulk source (sink) of the "particles" $k$, respectively. Thus, the subscript " $k$ " enumerates the corrosion defect and " $K$ " is the total number of different corrosion defects in the system. By definition $R_{k}(x, t) d x d t$ yields the number of defects $k$ (per $\mathrm{cm}^{2}$ ) with depths between $x$ and $x+d x$ that arise (or disappear) during the period of time between $t$ and $t+d t$, due to the transformation (repassivation, in the case of pits).

This new formulation of DFA affords considerable advantage over the previous treatment $[1,2,5]$ : we now possess a method of calculating the DF for a complicated system. To fulfill this task, we must solve the system of Equations (3) with the corresponding boundary and initial conditions.

$$
j_{k}=n_{k}(t) \text { at } x=0, t>0
$$


and

$$
f_{k}=f_{k 0}(x) \text { at } x>0, t=0
$$

where $f_{k 0}(x)$ is the initial distribution of defect $k$ [usually we can assume that $f_{k 0}(x)=0$ ), i.e., no damage exists at zero time] and $n_{k}(t)$ is the nucleation rate of the same defect [i.e, $n_{k}(t) d t$ is the number of stable defects $\left(\right.$ per $\left.\mathrm{cm}^{2}\right)$ that nucleate in the induction time interval between $\mathrm{t}$ and $t+d t]$.

Thus, because the defect propagation flux, $j_{k}$, must be non- negative (the depth of a corrosion event can only increase with time), the following, simplest numerical upwind finite difference scheme can be used for numerically solving Equations (3) - (5).

$$
f_{k, m}^{n+1}=f_{k, m}^{n}-\frac{\Delta t}{\Delta x}\left(j_{k, m}^{n}-j_{k, m-1}^{n}\right)+R_{k, m}^{n} \Delta t
$$

Here, we use the straightforward approach of choosing equally spaced points along both the t- and $\mathrm{x}$-axes: $x_{j}=x_{0}+m \Delta x, \quad m=0,1, \ldots, J$ and $t_{j}=t_{0}+n \Delta x, \quad n=0,1, \ldots, N$, and we denote $f_{k, m}^{n}=f_{k}\left(t_{n}, x_{m}\right)$. The values $f_{k, 0}^{n}$ and $f_{k, m}^{0}$ are calculated from the boundary and initial Conditions (4) and (5). Of course, it is assumed that we know (i.e. can calculate) fluxes, $j_{k}$, and sources, $R_{k}$, as functions of the spatial coordinates and time, and, in the general case, as functions of the unknown values of $f_{k}$.

In the simplest cases, it is even possible to obtain analytical solutions for the damage functions. As an example, let us consider the case of pitting corrosion under constant external conditions. In this case, we have two kinds of defect $(K=2)$ : active pits with the damage function, $f_{a}$, and passivated pits (i.e., those that have "died" through delayed repassivation) with the damage function, $f_{p}$. Here, and elsewhere in this paper, subscripts " $a$ " $(k=1)$ and " $p$ " $(k=2)$ denote active and passive pits, respectively.

Let us assume that the rate of pit propagation, $V$, depends only on the depth of the pit, $x$. Accordingly, for the flux of active pits, $j_{a}$, we have:

$$
j_{a}(x, t)=f_{a}(x, t) V(x)
$$


Generalization of this expression will be done later. However, it worth noting that the expression for the flux density cannot contain "diffusion terms", because pits can only increase their depth and the flux does not depend on the gradient in the pit population and hence on the differential damage function. By definition, the flux of passivated pits, $\mathrm{j}_{\mathrm{p}}$, is zero (i.e., these pits are "dead"). It is also evident that functions $R_{a}$ and $R_{p}$ must obey the relation $R_{p}=-R_{a}$, since the rate of appearance of passived pits must equal the rate of disappearance of active pits, due to passivation, at any given moment in time. If, in addition, we assume that the pit repassivation process obeys a first order decay law (the rate of passivation is proportional to the number of active pits), the function $R_{a}$ has the form

$$
R_{a}(x, t)=-\gamma f_{a}(x, t)
$$

where $\gamma$ is the delayed repassivation ("death") constant (i.e., the rate constant for repassivation of stable pits). The negative sign indicates that the number of active pits decreases due to repassivation. In the general case, $\gamma$ depends on the external conditions and, accordingly, on the depth of the pit, if significant potential and concentration drops exist within the cavity. In addition, $\gamma$ may also depend on time when the external conditions vary. However, in this example, we assume that $\gamma$ is a constant and hence that the probability of repassivation does not depend on pit depth. (This is clearly a gross over-simplification, since the probability of delayed repassivation is expected to increase with pit age, but this does not change the logic of the argument). Accordingly, the system of equations for calculating the DFs has the form

$$
\frac{\partial f_{a}}{\partial t}+\frac{\partial\left(V f_{a}\right)}{\partial x}=-\not f_{a}
$$

and

$$
\frac{\partial f_{p}}{\partial t}=\gamma f_{a}
$$

The boundary and initial conditions are then given as 


$$
V f_{a}=n(t) \text { at } x=0, t>0 \quad \text { and } \quad f_{a}=f_{p}=0 \quad \text { at } t=0
$$

The difference between Equations (9) and (10) is as follows: Equation (9) shows that the number of active pits can change due to the nucleation of new pits and the repassivation of existing (active) pits. In contrast, the number of dead pits can change only due to the repassivation of active pits (Equation 10).

An analytical solution to the boundary value problem formulated above yields

$$
f_{a}=\frac{\exp [-\gamma \theta(x)] n[t-\theta(x)]}{V(x)}
$$

and

$$
f_{p}=\frac{\gamma \exp [-\gamma \theta(x)] N[t-\theta(x)]}{V(x)}
$$

where

$$
\theta_{p i t}(x)=\int_{0}^{x} \frac{d x^{\prime}}{V\left(x^{\prime}\right)}
$$

is the age of a pit with depth $x$ and

$$
N(t)=\int_{0}^{t} n\left(t^{\prime}\right) d t^{\prime}
$$

is the number of stable pits $\left(\right.$ per $\mathrm{cm}^{2}$ ) that nucleate in the time interval between 0 and $t$.

It is important to note that, experimentally, only the sum of the damage functions for active and passive pits, $f=f_{a}+f_{b}$, is determined and, in many instances, the integral damage function, $\mathrm{F}=\mathrm{F}_{\mathrm{a}}+\mathrm{F}_{\mathrm{p}}$, only is measured. Accordingly, it is important, for practical reasons, to obtain the equation for the integral damage function. Note that the integral damage function, $\mathrm{F}$, corresponds to the number of remaining pits (active and passive) on a surface, as the surface is removed layer-by-layer in pre-established increments. From Equations (2), (12), and (13) we therefore have 


$$
F(x, t)=\exp [-\gamma \theta(x)] N[t-\theta(x)]
$$

According to the theory developed above, calculation of the damage functions requires the determination of three independent functions for each kind of corrosion defect, $k$ : The rate of defect nucleation, $n_{k}$, the flux density (growth rate) of the defect, $j_{k}$, and the rate of transition of one kind of defect to another, $R_{k}$, (for example, the transition of an active pit into a passive pit or the transition of a pit into a crack). Below, we will discuss briefly the feasibility of calculating each of these three functions from first principles.

In many practical cases, it is possible to assume that all pits on a given surface nucleate during an initial period of time that is much less than the observation time, $t$, or the service life of the system, $\mathrm{t}_{\mathrm{s}}$ (instantaneous nucleation). For example, for the case of the pitting corrosion of aluminum in tap water, practically all of the pits were found to nucleate within the first two weeks [9]. Under these conditions, the total number of nucleated stable pits, as a function of time, can be simply represented as

$$
N(t)=N_{0} U_{+}(t)
$$

where $N_{0}$ is the maximum number of the stable pits $\left(\right.$ per $\left.\mathrm{cm}^{2}\right)$ that can exist on the metal surface and $U_{+}(t)$ is the asymmetrical unit function $\left(U_{+}=0\right.$ at $t \leq 0$ and $U_{+}=1$ at $\left.t>0\right)$.

If pit nucleation cannot be regarded as being "instantaneous", the simplest assumption concerning the pit nucleation rate, $n(t)=d N / d t$, is that $n(t)$ is proportional to the number of available sites, $N_{0}-N(t)$ [10], which yields.

$$
N(t)=N_{0}\left[1-\exp \left(-t / t_{0}\right)\right]
$$

where $t_{0}$ is some characteristic time that depends on the corrosion potential, temperature, and electrolyte composition.

A more general and sophisticated calculation of the pit nucleation rate can be made in the following way. Although an extensive database does not exist to support this 
position, it is postulated that the rate of nucleation of stable pits, $n=d N / d t$, is related to the rate of nucleation of metastable pits, $n_{M P}=(d N / d t)_{M P}$, by

$$
n(t)=\frac{d N}{d t}=\zeta\left(\frac{d N}{d t}\right)_{M P}
$$

where parameter $\zeta$ is termed the survival probability [10-12]. This parameter can be measured experimentally. Thus, for example, for Type 304L stainless steel in $\mathrm{NaCl}$ solution, the experimentally measured survival probability has a value of the order of $10^{-2}$ [12].

For calculating the rate of nucleation of metastable pits, the Point Defect Model (PDM) can be used [13-17]. On the basis of this model, it can be shown that total number of nucleated pits can be described by the following expression [5]

$$
N(t)=N_{0} \operatorname{erfc}\left(\frac{a}{t}+b\right) / \operatorname{erfc}(b)
$$

Parameters $a$ and $b$ specifically depend on temperature, $\mathrm{pH}$, activity of halide ion, and the metal potential, but do not depend on the induction time. These dependencies can be found in the original work $[5,13-17]$ or in the Appendix to this article. To the best of our knowledge, the PDM is the only model that establishes the connection between pit nucleation rate and the external conditions. For example, in the empirical Equation (18), the connection between the parameter, $t_{0}$, and the external conditions ( $\mathrm{pH}$, potential, etc.) is not specified. It is interesting to note that, if the observation time, $t$, satisfies the condition $t>>a\left(b+\sqrt{b^{2}+4}\right) / 2$, practically all pits nucleate during a very short period of time at the beginning of experiment, i.e. the PDM predicts the limiting case of instantaneous nucleation [5].

However, it is also important to note that the original PDM describes pit nucleation for the case when the external conditions do not depend on time. This restriction can be a serious impediment in predicting the pit nucleation rate under practical conditions, for example, when seasonal changes in temperature and wetness are 
observed for a system that is subject to atmospheric corrosion. However, the PDM is easily generalized for the case of variable external conditions, as described in the Appendix.

The quantitative description of pit (or crack) growth can be regarded as one of the key problems in predicting corrosion damage in many practical systems. This follows from the fact that the calculated corrosion damage that is based only on this (growth) stage can be compared with experiment, in many limiting cases. For example when all pits nucleate "instantaneously", or when the induction time for pit nucleation is much smaller than the observation time, it is possible to ignore the initial stage of pit nucleation when estimating the damage. In addition, if the probability of survival of a corrosion defect is sufficiently high, we must take into account the possibility that a stable corrosion defect (pit or crack) nucleates immediately after the start of operation and propagates without repassivation. In any case, calculations based only on the growth stage yield the most conservative estimate of the service life, $t_{s, \min }$, of the system. We can be sure that, if calculation of the service life is based on growth alone, the real service life, $t_{s,}$ will at least be not less than $t_{s, \min }$.

It is well known from both experiment [18] and theory [19] that the dependence of the characteristic dimension of a corrosion cavity (for example, cavity depth, $L$ ) on time, $t$, can be expressed by a simple equation of the following form

$$
L=k t^{m}
$$

where $k$ and $m$ are empirical constants, and, usually, $m \leq 1$. However, this dependence of $L$ on $t$ cannot be used directly in mathematical calculations for small times, because of the non-physical limit

$$
V=\frac{d L}{d t}=k m t^{m-1} \rightarrow \infty, \quad \text { at } t \rightarrow 0 \text { and } m<1
$$

This is why, instead of Equation (21), we use the following interpolation equation for pit propagation rate 


$$
V=\frac{d L}{d t}=V_{0}\left(1+t / t_{0}\right)^{n}
$$

where $\mathrm{n}=\mathrm{m}-1$ and $\mathrm{t}_{0}$ are constants, and $V_{0}$ is the initial, finite rate of pit propagation. Equation (23) yields $V=V_{0}$ at $t / t_{0}<<0$ and $V=V\left(t / t_{0}\right)^{n}$ at $t>>t_{0}$.

It is important to note that, in many cases, the period of time over which the approximation

$$
V(x) \approx V_{0}=\text { constant }
$$

is valid can be comparable with the observation time (or even with the service life of the system). The reason is that corrosion is, generally speaking, a slow process and under real, practical conditions, values of the critical pit depth of the system, $x_{c r}$, (e.g., wall thickness of a pipe) and typical service life, $t_{s}$, impose significant restrictions on the values of the initial and average corrosion current densities and, thus, on the potential and concentration drops that might be observed in a corrosion cavity [20]. Thus, as follows from Faraday's law, if $x_{c r}$ does not exceed the order of $1-10 \mathrm{~mm}$, and if the order of $t_{s}$ is not less than 1 year, the initial corrosion current densities in real pits cannot exceed values of $10^{-4}-10^{-3} \mathrm{~A} / \mathrm{cm}^{2}$, with the understanding that the polarization curve (corrosion current density vs. potential) does not change as the pit propagates [20]. Such low current densities can arise only from relatively low values of the potential and concentration drops in open pits. In particular, it is shown that if localized corrosion takes place in an electrolyte having a conductivity of the order of that of sea water, it is possible to neglect the potential drop in an open corrosion cavity and hence it is possible to regard the rate of pit propagation as being constant under constant environmental conditions. The same conclusion holds for the pitting of $316 \mathrm{~L}$ stainless steel for practically any composition of the external electrolyte. This issue is discussed in greater depth in Ref. 20.

As noted above, we assume that the repassivation process obeys a first-order decay law [see Equation (8)]. The repassivation constant, $\gamma$, is, in general, a function of 
the external conditions, including the corrosion potential, temperature, and electrolyte composition. Generally speaking, $\gamma$ is also a function of the depth of the pit, $x$, because the local potential in the solution at the cavity surface depends on $x$, i.e. $\gamma$ might be a function of both the spatial coordinates and time. Of course, if the potential and concentration drops inside the corrosion cavity are insignificant during pit propagation, it is possible to neglect changes in parameter $\gamma$ (see above). In the current investigation, we assume, for simplicity, and hence to facilitate the development of analytical expressions for the damage functions $f_{a}$ and $f_{p}$, that $\gamma$ is a constant [1]. Of course, the value of this constant still depends on the external conditions, such as potential, $\mathrm{pH}$, and concentration of aggressive species in the bulk electrolyte. However, we can imagine the situation when the potential and concentration drops inside the pit are significant and timedependent (for example, in extremely dilute solutions or in closed pits); in which case the probability of repassivation will be a function of pit depth and hence pit age. It also can be that active pits cannot exist if the potential, E, at the pit internal surface is less then some critical value, $\mathrm{E}_{\mathrm{cr}}$. Accordingly, if the value of $\mathrm{E}_{\mathrm{cr}}$ is reached at some pit depth, $\mathrm{x}_{\mathrm{cr}}$, active pits passivate and cannot penetrate further into the metal. Accordingly, Equation (16) cannot be used.

Issues and Concerns: $\quad$ None

\section{III-5.2 Task: Generalization of PDM to Describe Passivity Breakdown (where the external conditions vary with time)}

\section{Task Status}

In the original version of PDM [13-17], the pit nucleation rate was obtained from the criterion for pit initiation:

$$
\left(J_{c a}-J_{m}\right) \times(t-\tau) \geq \xi
$$

where $J_{c a}$ is the cation vacancy flux in the barrier layer of the passive film at the metal/barrier layer interface, $J_{m}$ is the rate of annihilation of the cation or metal vacancies 
at the same location, $t$ is the observation time, $\tau$ is the time of dissolution of the pit cap until rupture, and $\xi$ is the critical "areal" concentration $\left(\# / \mathrm{cm}^{2}\right)$ of condensed vacancies that are required for the separation of the barrier layer from the substrate metal. This latter parameter can be obtained from the potential sweep rate dependence of the apparent breakdown potential and can also be calculated from structural arguments [27, 28]. However, Criterion (A-1) is valid only if all external parameters (temperature, electrolyte composition, $\mathrm{pH}$, corrosion potential, etc.) do not depend on time, and, accordingly, $J_{c a}$, $J_{m}$, and $\xi$ are constants. In the general case, when the external conditions are timedependent, instead of Criterion (A-1) we must use the following criterion:

$$
\int_{\tau}^{t}\left[\left(J_{c a}\left(t^{\prime}\right)-J_{m}\left(t^{\prime}\right)\right] d t^{\prime} \geq \xi(t)\right.
$$

Note that $\xi$ corresponds to the number of cation vacancies per unit area condensed on the cation sublattice on the film side of the metal/barrier layer interface or the number of metal vacancies condensed on the metal lattice on the metal side of the interface, depending upon whether vacancy condensation occurs on the cation sub-lattice of the film or on the metal lattice, respectively. In any event, the value of $\xi$ is of the order of $(2-3) \times 10^{15} \mathrm{~cm}^{-2}$, depending upon the crystallographic faces involved [27, 28]. The principal time-dependence of $\xi$ most likely arises from expansion/contraction of the lattice as the temperature changes, in which case $\xi=\xi_{0} \exp \left[-2 \hat{\alpha}\left(T-T_{0}\right)\right]$, where $\hat{\alpha}$ is the coefficient of linear thermal expansion, $T$ is the Kelvin temperature, $T_{0}$ is the reference temperature, and $\xi_{0}$ is the critical vacancy concentration at $T_{0}$. For example, we evaluate $\exp \left[-2 \hat{\alpha}\left(T-T_{0}\right)\right]$ for nickel (a simple, ccp metal with $\hat{\alpha}=1.3 \times 10^{-5} \mathrm{~K}^{-1}$ [29]) to be 0.9981 for $T=373.15 \mathrm{~K}\left(100{ }^{0} \mathrm{C}\right)$ and $T_{0}=298.15 \mathrm{~K}\left(25{ }^{0} \mathrm{C}\right)$, assuming that vacancy condensation occurs on the metal lattice, from which we conclude that $\xi$ can be considered to be quasi-constant. However, this may not be the case for passivity breakdown on a liquid metal, such as gallium [30], because of the much larger coefficient of expansion. 
In previous work [27, 28], $J_{m}$ has been estimated from $J_{m} \leq i_{\text {passive }}^{c} / n F$, where $i_{\text {passive }}^{c}$ is the steady-state passive current density at the point of film breakdown, $n$ is the number of electrons involved in the annihilation reaction, and $F$ is Faraday's constant. The inequality arises, because not all of the current may be carried by cation vacancies; indeed, in n-type passive films, such as those that form on iron and aluminum, where the principal point defects are either oxygen vacancies or metal interstitials, or both, the current density due to the flux of cation vacancies might be only a small fraction of the total current density. If the transport number of the cation vacancy $\left(t_{c v}\right)$ in the barrier layer is known by independent experiment, then the value of $J_{m}$ can be established precisely as $J_{m}=t_{c v} i_{\text {passive }}^{c} / n F$, where $i_{\text {passive }}^{c}$ is the passive current density at the point of breakdown in the absence of a redox couple in the solution. In any event, methods have been developed for determining the rate of annihilation cation vacancies at the metal/barrier layer interface, primarily by analyzing electrochemical impedance data [31], so that $J_{m}$ may be determined by independent experiment for any given set of external conditions. This leaves only $J_{c a}$ in Equation (A-2) to be determined by independent experiment, in order to affect a completely deterministic prediction of metastable pit nucleation rate. The rate constant for cation vacancy generation at the barrier layer/solution interface is readily determined using EIS and, for nickel, is found to be of the form $k=k_{0}+\mathrm{A}_{\left[\mathrm{Cl}^{-}\right]^{p}}$ [17], where $k_{0}$ is the rate constant for chloride free solutions and $A$ and $p$ are constants. For nickel in $\mathrm{NaCl}$ solutions [31], we have recently determined that $p=1$; that is, cation vacancy generation at the barrier layer/solution interface for passive nickel is first order in chloride concentration, a finding that is consistent with the Point Defect Model.

On any real surface, a large number of potential breakdown sites exist corresponding to a distribution in the properties of the "weak spots". Thus, examination of data reported by Shibata [32] and Fratesi [33], among others, suggests that the breakdown voltage is nearly normally distributed. The PDM assumes that the breakdown sites with respect to the distribution of diffusivity of cation vacancies is described approximately by a normal distribution function [17], i.e. 


$$
\frac{d N(D)}{d D}=-A \exp \left[-\frac{(D-\bar{D})^{2}}{2 \sigma_{D}^{2}}\right]
$$

Here, $N(D)$ is the number of breakdown sites (per $\mathrm{cm}^{2}$ ) that have diffusivities larger than $D$, and $\bar{D}$ and $\sigma_{D}$ are the average value and the standard deviation, respectively, of the diffusivity for the population of the breakdown sites. The negative sign in Equation (A3 ) means that $N(D)$ decreases with increasing $D$. Parameter $A$ does not depend on $D$, so that normalization of the diffusivity distribution using the condition $N(0)=N_{t o t}$, where $N_{t o t}$ is the total number of breakdown sites $\left(\right.$ per $\mathrm{cm}^{2}$ ), yields

$$
N(D)=N_{t o t} \operatorname{erfc}\left(\frac{D-\bar{D}}{\sqrt{2} \sigma_{D}}\right) \operatorname{erfc}\left(-\frac{\bar{D}}{\sqrt{2} \sigma_{D}}\right)
$$

The cation vacancy flux density, $J_{c a}$, is proportional to the diffusion coefficient of vacancies, $D$, i.e.

$$
J_{c a}=D B,
$$

where the function $B$ depends on the on the external conditions (applied voltage, $V_{a p p}$, temperature, $T$, chloride activity, $a_{x}$, etc.). Thus, for the case of passivity breakdown in chloride solutions, the PDM yields:

$$
B=\hat{a} u^{-\chi / 2} \exp \left(\frac{\chi F \alpha V_{a p p}}{2 R T}\right) a_{x}^{\chi / 2}
$$

where $\alpha$ is the polarizability of the film/solution interface (i.e., dependence of the potential drop across the barrier layer/solution interface on the applied potential), $\chi$ is the cation oxidation state in the barrier layer, $R$ is the gas constant, and $F$ is Faraday's constant. In turn, parameters $\hat{a}$ and $u$ are defined as [13] 


$$
\hat{a}=\chi(F \varepsilon / R T)\left[N_{A} / \Omega\right]^{1+\chi / 2} \exp \left(-\Delta G_{S}^{0} / R T\right)
$$

and

$$
u=\frac{N_{A}}{\Omega} \exp \left(\frac{\Delta G_{A}^{0}-\beta F p H-F \phi_{f / s}^{0}}{R T}\right)
$$

where $\varepsilon$ is the field strength in the film, $\Omega$ is the mole volume per cation, $\Delta G_{S}^{0}$ is the change of Gibbs energy for the Schottky-pair reaction (Null $\rightarrow V_{M}^{\chi^{\prime}}+(x / 2) V_{o}^{\bullet}$, assuming that this is the cation vacancy generation process - see Ref. 20), $\beta$ is the dependence of the potential drop across the film/solution interface on $\mathrm{pH}, \phi_{\mathrm{f} / \mathrm{s}}^{0}$ is a constant (potential drop at the film/solution interface for $V_{a p p}=0$, and $p H=0$ ), and $\Delta G_{A}^{0}$ is the standard Gibbs energy change for the chloride absorption reaction $V_{O}^{\bullet \bullet}+C l_{a q}^{-} \rightleftharpoons$ $\mathrm{Cl}_{O}^{\bullet}\left(\mathrm{V}_{O}^{\bullet \bullet}\right.$ is an oxygen vacancy in the film and $\mathrm{Cl}_{O}^{\bullet}$ is a chloride anion occupying a surface oxygen lattice site). A detailed discussion of these parameters is available in the original papers [13-17]. The assumption of a normal distribution of the breakdown sites with respect to $D$ results in a near-normal distribution in the breakdown voltage and a left-acute distribution in the induction time, in agreement with experiment [14-17].

Let us denote by $D_{0}$ the diffusivity of a cation vacancy at temperature $T_{0}$. We can calculate $D$ at any other temperature by using the relation

$$
D=D_{0} \omega
$$

where

$$
\omega=\exp \left[-\frac{E_{D}}{R}\left(\frac{1}{T}-\frac{1}{T_{0}}\right)\right]
$$

and $E_{D}$ is the energy of activation for diffusion. 
Substituting Equations (A-5) and (A-9) into Equation (A-2) yields the following criterion for meta-stable pit nucleation as

$$
D_{0} \geq D_{c r}(t)=\frac{\xi+\int_{\tau}^{t} J_{m}\left(t^{\prime}\right) d t^{\prime}}{\int_{\tau}^{t} \omega B\left(t^{\prime}\right) d t^{\prime}}
$$

This criterion has to be understood in the following way. Let us assume that, at $t=0$ (at the beginning of corrosive attack), a potential breakdown site is characterized by a cation vacancy diffusivity of $D_{0}$. Criterion (A-11) states that the nucleation of metastable pits occurs within the observation time, $t$, on those and only on those sites that have $D_{0} \geq$ $D_{c r}(t)$.

Substitution of Equation (A-9) into Equation (A-4) yields an expression for the number of metastable pits (per $\mathrm{cm}^{2}$ ) that nucleate during the period of time $(0, t)$.

$$
N(t)_{M P}=N_{t o t} \operatorname{erfc}\left(\frac{D_{c r}(t)-\bar{D}_{0}}{\sqrt{2} \sigma_{D_{0}}}\right) / \operatorname{erfc}\left(-\frac{\bar{D}_{0}}{\sqrt{2} \sigma_{D_{0}}}\right)
$$

Subscript "0" in Equation (A-12) means that the values are calculated at temperature $T_{0}$, i.e. at $t=0$. Under constant external conditions, Equation (31) reduces to the previously developed expression [5]

$$
N(t)_{M P}=N_{t o t} \operatorname{erfc}\left(\frac{a}{t-\tau}+b\right) / \operatorname{erfc}(b)
$$

where $a=\xi /\left(B \sqrt{2} \sigma_{\mathrm{D}_{0}}\right)$ and $b=\left(J_{m} / B-\bar{D}\right) /\left(\sqrt{2} \sigma_{D_{0}}\right)$.

By differentiating Equation (A-13), we obtain an expression for calculating the rate of nucleation of metastable pits: 


$$
n_{M P}=\frac{d N_{M P}(t)}{d t}=\frac{\sqrt{2}}{\operatorname{erfc}\left(-\bar{D}_{0} / \sqrt{2} \sigma_{D_{0}}\right) \sqrt{\pi} \sigma_{D_{0}}} \exp \left[-\frac{\left(D_{c r}(t)-\bar{D}_{0}\right)^{2}}{2 \sigma_{D_{0}}^{2}}\right] \frac{d D_{c r}(t)}{d t}
$$

where, in accordance with Equation (A-11),

$$
\frac{d D_{c r}(t)}{d t}=\frac{d \xi / d t+J_{m}(t)}{\int_{\tau}^{t} \omega B\left(t^{\prime}\right) d t^{\prime}}-\frac{\omega B(t)\left(\xi(t)+\int_{\tau}^{t} J_{m}\left(t^{\prime}\right) d t^{\prime}\right)}{\left[\int_{\tau}^{t} \omega B\left(t^{\prime}\right) d t^{\prime}\right]^{2}}
$$

For the case when the external conditions do not depend on time, we have a more simple expression

$$
n_{M P}=\frac{N_{t o t} 2 a}{\operatorname{erfc}(b) \sqrt{\pi}} \frac{\exp \left[-\left(\frac{a}{t-\tau}+b\right)^{2}\right]}{(t-\tau)^{2}}
$$

which we previously derived [5].

It is important to note that all expressions for $N_{M P}$ or $n_{M P}$ are valid only for $t>\tau$. If $t \leq \tau$, we simply have $N_{M P}=0$ and $n_{M P}=0$. However, for the majority of cases, the observation time, $t$, is much greater than $\tau$, and accordingly it is possible to let $\tau \approx 0$. Under these conditions, Equations (A-13) and (19) yield Equation (20) (assuming that $N_{0}=\zeta N_{t o t}$, which has been previously used for estimating the number of stable pits nucleated under constant external conditions.

Issues and Concerns: $\quad$ None

\section{III-5.3 Developing a Relationship Between Deterministic and Statistical Methods for Predicting the Progression of Corrosion Damage}


In this section of the project, we explore and develop the relationship that exists between deterministic and statistical methods for predicting the progression of corrosion damage. Our objective is to show mathematically that there exists a close correspondence between Damage Function Analysis (DFA), which has been described at some length in the first part of this paper and Extreme Value Statistics (EVS). The latter technique has been used extensively to extrapolate damage (maximum pit or crack depth) from small samples in the laboratory to larger area samples in the field. Furthermore, DFA provides a means of calculating the central and scale parameters and their timedependencies in EVS from first principles and hence represents a unification of the two philosophies.

\section{$\underline{\text { Task Status }}$}

From the statistical point of view, all distributed properties of the system are completely determined by a Cumulative Distribution Function (CDF), $\Phi(x)$. By definition, $\Phi(x)$ is the probability that the depth of a randomly selected pit is $\leq x$. We will postulate that the pit distribution on the metal surface is uniform. Accordingly, the total number of nucleated pit in the whole system is $\mathrm{SN}(\mathrm{t})$, where $\mathrm{S}$ is the area of the system and, from the definition of the integral damage function, $F$, the number of pits that have the depth $\leq x$, is $\mathrm{S}[\mathrm{N}(\mathrm{t})-\mathrm{F}(\mathrm{x}, \mathrm{t})]$. Accordingly, by definition of probability we have

$$
\Phi(x, t)=\frac{S[N(t)-F(x, t)]}{S N(t)}=1-\frac{F(x, t)}{N(t)}
$$

We see that the cumulative distribution function for a given observation time, $\Phi(x, t)$, can be calculated if we know (can calculate) the integral damage function of the system [note that the number of nucleated stable pits, $N(t)$ simply equals $F(0, t)]$. This relation can be regarded as being the bridge between the statistical and deterministic approaches for estimating the accumulation of localized corrosion damage on a surface.

Let us calculate, for example, the failure probability, $\mathrm{P}_{\mathrm{f}}$, of a system. By definition, $\mathrm{P}_{\mathrm{f}}$, is the probability that at least one corrosion event in any form (pit, crevice, stress corrosion crack, or fatigue crack) reaches a depth, $\mathrm{x}$, at a given observation time, $\mathrm{t}$, 
where $\mathrm{x}$, in this case, is the critical dimension. It is evident that the probability of a corrosion event not achieving the depth $x$ at the tine, $\mathrm{t}$, is $\Phi(x, t)$. Accordingly, the probability that none of the corrosion events will reach the depth of $x$ is, $\Phi(x, t)^{S N(t)}$ where $S$ is the surface area of the system and, finally, the probability of failure is

$$
P_{f}(x, t)=1-\{1-F(x, t) / N(t)\}^{S N(t)}
$$

Using the well known limit relation, $\lim (1+a / x)^{x} \quad \cdots \exp (a)$ as $x \rightarrow \infty$, and by assuming that $\mathrm{F}(\mathrm{x}, \mathrm{t}) / \mathrm{N}(\mathrm{t})<<1$ and $S N(t)$ is a large number, we can rewrite Equation (26) in the form

$$
P_{f}(x, t)=1-\exp \{-S F(x, t)\}
$$

As has been shown experimentally, in many practical cases, the asymptotic behavior (for large values of $x$ ) of the CDF can be described by the exponential relationship $[8,23]$.

$$
\Phi(x, t)=1-\exp [-(x-u) / \alpha]
$$

where $u$ is the central parameter (the most frequent value) and $\alpha$ is the scale parameter, which defines the width of the distribution.. Accordingly, as follows from Equation (28), the extreme value distribution (EVD), $\Psi(x, t)$ (the probability that the largest value of pit depth $\leq x$ ) is described by a double exponent (Gumbel Type I extreme value distribution) in the form [22].

$$
\Psi(x, t)=1-P_{f}=\exp [-\exp (-y)]
$$

where

$$
y=(x-h) / \alpha
$$


and $h=u+\alpha \ln S$.

In many applications, the Type 1 distribution has been claimed to account for the statistical nature in the observed behavior of corrosion systems [8,9,21,23]. Specifically, the Type I extreme value distribution is used for extrapolating corrosion damage measured on a series of small samples of area, $s$, to a large system (for example, tanks or pipe lines) with area, $S$ [24]. In this case, the probability of failure can be estimated from the relation

$$
P_{f}(x, t)=1-\exp \{-\exp [-(d-[h+\alpha \ln (S / s)]) / \alpha)]
$$

where $d$ is the wall thickness and parameters $h$ and $\alpha$ are measured by using the small samples.

It is interesting to determine whether Damage Function Analysis (DFA) can predict the behavior of the extreme value distribution. For simplicity, we will consider the limiting, but real, case when the observation time is much longer than the induction time for the majority of nucleated stable pits ("instantaneous" nucleation conditions). Experimentally determined extreme value distributions for pits have been reported for these conditions [9]. In addition, we also assume that the pit propagation rate is so low that it can be considered to occur at a constant rate, $V$. Under these conditions, as follows from Equations (16), (17), and (25), the Cumulative Distribution Function is given by

$$
\Phi(x, t)=1-\exp (-\gamma x / V) U_{+}(t-x / V)
$$

As noted by many sources $[8,21]$, an important characteristic of the unlimited CDF [see Equation (28)] is that it implies a small, but finite, probability of observing a pit of exceptional depth. The CDF given by Equation (28) was obtained from a conservation law and naturally it is free from that weakness (there are no pits for $x>V t$ ). For $x \leq V t$ the CDF given by Equation (32) coincides with the CDF described by Equation (28), if we choose $\alpha=V / \gamma$ and $u=0$, i.e. parameters $\alpha$ and $u$ do not depend on time. However, experiment shows that parameters $\alpha$ and $u$ are generally functions of time, and, 
particularly, the central parameter, $u$ is positive [7-9]. Moreover, for short periods of time, we can neglect the repassivation of pits, i.e. let $\gamma=0$ in Equation (32). In this case, Equation (32) reduces to a step function. However, experimentally observed extreme value distributions with $u>0$ for systems where the period of time over which damage has developed is of order of $10^{2}-10^{3}$ hours [7], i.e. for periods that are much lower than the service life, $t_{s}$, of many practical systems, leaves little doubt that a distribution exists in the cavity growth rate, as alluded to earlier in this paper.

Because, under these limiting conditions, we neglect the nucleation stage of pit life ("instantaneous" nucleation) and because we also neglect the repassivation of pits, the only explanation of the inconsistency noted above must arise from the propagation of pits. In other words, Equation (7) cannot be used, in the general case, for describing the flux density of pits. It has been tacitly assumed above that the rate of pit propagation is unequivocally determined by its depth and by the external conditions, i.e. there is no distribution in pit propagation rate for pits with equivalent depth. However, it is well known that the morphology of pits on any given surface can vary significantly, with some shapes favoring more rapid mass and charge transfer, and hence greater propagation rate [8]. In addition, some pits will initiate at metallurgical features that may favor more rapid propagation, e.g. $\mathrm{MnS}$ inclusion [8]. The distribution in pit propagation rate might also be explained, for example, by the spatial distribution in electrochemical activity of the anodic and cathodic sites on the corroding surface [7].

In this article, we will assume, for simplicity, that the pit propagation rate does not change significantly during the propagation stage. This assumption can be justified for many real systems [20]. In generalizing DFA, for this case, we assume that the pits that propagate at a constant rate, $\mathrm{v}$, nucleate in accordance with the equation.

$$
n(t)=\int_{0}^{\infty} \lambda(t, v) d v
$$

Here, the function $\lambda(t, v)$ yields the number of pits with propagation rates between $v$ and $v+d v$, that nucleate in the period of time between $t$ and $t+d t\left(\mathrm{per}^{2}\right)$. For the total number of nucleated pits, we have by analogy 


$$
N(t)=\int_{0}^{\infty} \Lambda(t, v) d v
$$

where $\Lambda(t, v)$ yields the total number of pits with propagation rates between $v$ and $v+d v$ that are nucleated during the observation time, $t,\left(\right.$ per $\left.\mathrm{cm}^{2}\right)$. Finally, it is evident that

$$
\Lambda(t, v)=\int_{0}^{t} \lambda(\tau, v) d \tau
$$

Equation (7), for the flux density of active pits in the absence of the distribution in pit propagation rate, together with $V(x) \approx$ constant, can be rewritten in the following form [see Equation (12)]

$$
j_{a}(x, t)=f_{a}(x, t) V(x)=\exp (-\gamma \theta) n(t-\theta)=\exp (-\gamma x / V) n(t-x / V)
$$

and hence, when a distribution in the pit propagation rate is present, this expression can be generalized as

$$
j_{a}(x, t)=\int_{0}^{\infty} \exp (-\gamma x / v) \lambda(t-x / v, v) d v
$$

Under these conditions, the solutions of transport Equation (3) have the form

$$
\begin{aligned}
& f_{a}=\int_{0}^{\infty} \frac{\exp (-\gamma x / v) \lambda(t-x / v, v)}{v} d v \\
& f_{p}=\int_{0}^{\infty} \frac{\gamma \exp (-\gamma x / v) \Lambda(t-x / v, v)}{v} d v
\end{aligned}
$$


and

$$
F=\int_{0}^{\infty} \exp (-\gamma x / v) \Lambda(t-x / v, v) d v
$$

Let us assume that the distribution in pit propagation rate does not depend on time, i.e.

$$
\lambda(t, v)=n(t) \psi(v)
$$

and, correspondingly,

$$
\Lambda(t, v)=N(t) \psi(v)
$$

where the function, $\psi(\mathrm{v})$, satisfies the evident normalization condition

$$
\int_{0}^{\infty} \psi(v) d v=1
$$

Accordingly, we have for the integral damage function and the cumulative damage function the following expressions

$$
F(x, t)=\int_{0}^{\infty} N(t-x / v) \exp (-\gamma x / v) \psi(v) d v
$$

and

$$
\Phi(x, t)=1-\int_{0}^{\infty} N(t-x / v) \exp (-\gamma x / v) \psi(v) d v / N(t)
$$

respectively. In particular, for the case of instantaneous nucleation, we have 


$$
\Phi(x, t)=1-\int_{x / V}^{\infty} \exp (-\gamma x / v) \psi(v) d v
$$

To move further, we must assume a distribution function, $\psi(\mathrm{v})$, for the pit growth rate. For our purposes, it is most convenient to approximate $\psi(v)$, by Laplace's distribution function [25]

$$
\psi(\mathrm{v})=\frac{\exp (-|\mathrm{v}-\mathrm{V}| / \beta)}{2 \beta}
$$

with the mean pit propagation rate, $\mathrm{V}$, and dispersion $\sigma^{2}=2 \beta^{2}$. Strictly speaking, Distribution (47) satisfies the normalization condition $\int_{-\infty}^{\infty} \psi(v) d v=1$, but not Condition (43). The proper form of Distribution (47) that satisfies Conditions (43) is

$$
\psi(\mathrm{v})=\frac{\exp (-|\mathrm{v}-\mathrm{V}| / \beta)}{2[\beta-\exp (-\mathrm{V} / \beta)]}
$$

However, for $V / \beta \geq 2$, Distributions (47) and (48) practically coincide.

Generally, values for the kinetic parameters $V$ and $\beta$ (along with parameters that described pit nucleation and repassivation) can be found from independent experiments or might even be estimated from first principles. In any event, all unknown parameters can also be determined by fitting Equations (44) or (27) to the experimentally measured CDF or EVD functions, as is customary, by applying a purely statistical approach. However, the application of DFA for estimating localized corrosion damage has real advantages over the purely statistical approaches. Thus, DFA yields the connection between the CDF and the physical parameters that determine the accumulation of damage. This connection allows us to reduce the number of fitting parameters for the CDF, to predict the time dependencies of these parameters, and to predict the dependencies of 
some fitting parameters on the external conditions (corrosion potential, temperature, electrolyte composition, etc.).

As an example, let us consider the extrapolation of corrosion damage into the future for the system that has been investigated in Ref. 7: corrosion of manganese steel in $\mathrm{CO}_{2-}$ acidified seawater. Figure III-5.1 shows experimental extreme value distributions for pit depth measured for six different times (from $t_{1}=336$ hours to $t_{6}=1176$ hours). We will assume that we know nothing about the kinetic parameters for this system. Nevertheless, we will try to predict the propagation of the damage into the future by using only data obtained at $t=t_{1}$ and by using only the simplest, qualitative assumption concerning the damage propagation process.

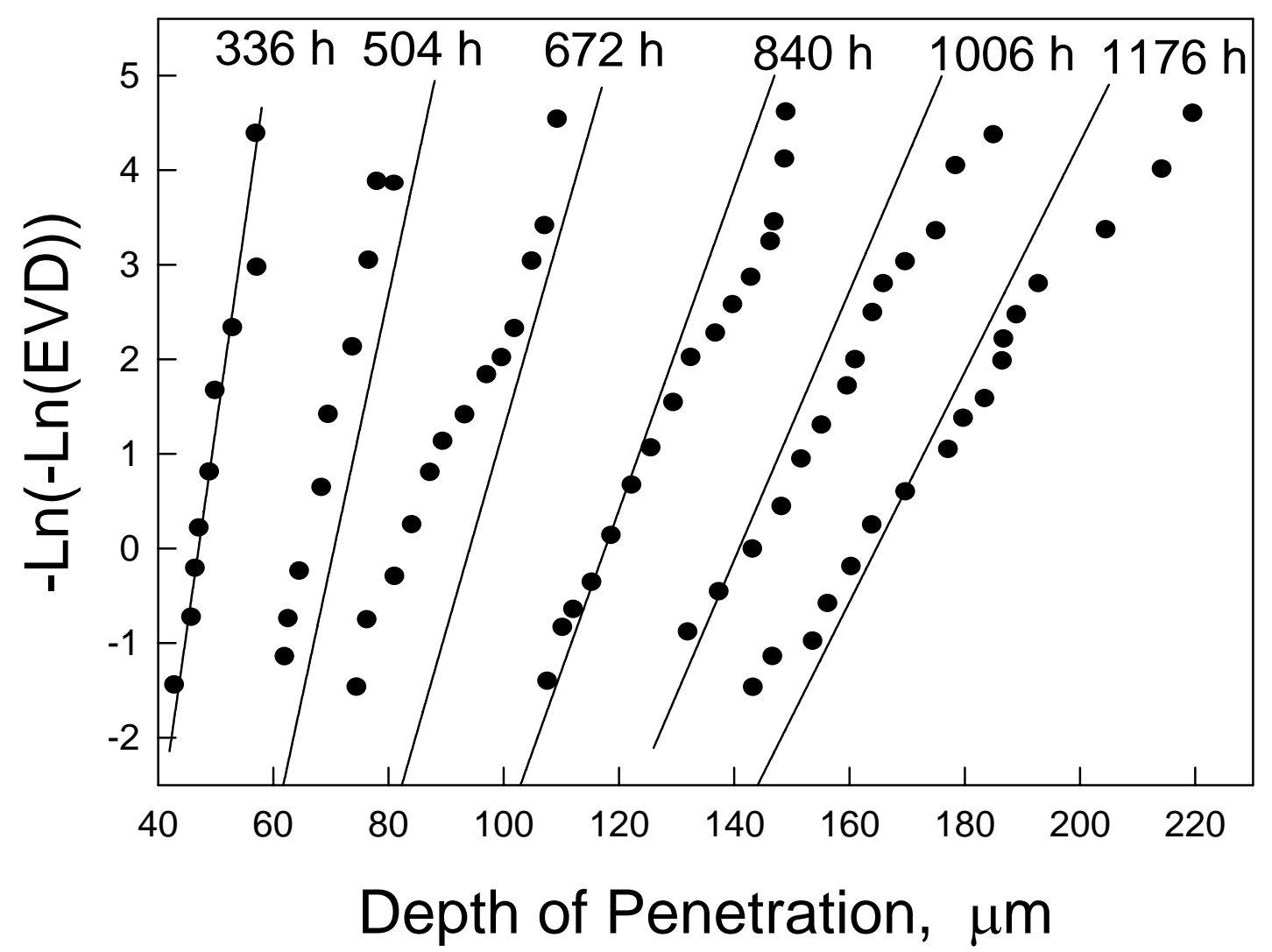

Figure III-5.1 Examples of predicted damage functions in pit depth for the pitting corrosion of aluminum in chloride solutions for $E_{\text {corr }}=-0.384 \mathrm{~V}_{\text {she }},\left[\mathrm{Cl}^{-}\right]=0.002 \mathrm{M}, \mathrm{pH}=7$, and $\gamma=3$ year [1]. 
First of all, we assume that the induction time for pit nucleation is much smaller than $t_{l}$ and, accordingly, that pit nucleation can be regarded as being instantaneous. This assumption is reasonable, if the environment is reasonably aggressive (high chloride concentration) and the exposure time is large, as is the case in this example. For example, many $\mathrm{Fe}-\mathrm{Cr}$ alloys display pit induction times of the order of $10^{2} \mathrm{~s}$ for commonly encountered environmental conditions [14]. We will also assume that $t_{l}$ is much smaller than the service life for normal structures made from this steel, and, accordingly, it is possible to neglect pit repassivation for $t \leq t_{1}$.

Substitution of Equation (47) into Equation (44) with $N(t)=N_{0} U_{+}(t)$ (instantaneous nucleation) and $\gamma=0$ (no pit repassivation) yields:

$$
F(x, t)=N_{0}\left[1-\frac{1}{2} \exp \left(\frac{x / t-V}{\beta}\right)\right] \quad \text { for } x \leq V t
$$

and

$$
F(x, t)=\frac{N_{0}}{2} \exp \left(\frac{V-x / t}{\beta}\right) \quad \text { for } x>V t
$$

Because we are interested in the behavior of the CDF for large values of $x$, we obtain the following expression for the EVD

$$
\Psi(x, t)=1-P_{f}=\exp \left[-\frac{S N_{0}}{2} \exp \left(\frac{V-x / t}{\beta}\right)\right]
$$

which absolutely coincides with the Type I extreme value distribution [Equations (29) and (30)] if we choose

$$
\alpha=\beta t \quad \text { and } \quad h=\left[V+\beta \ln \left(0.5 S N_{0}\right)\right] t
$$

The reader will note that Equation (51) does not contain parameters related to the nucleation of damage or to delayed repassivation, because we have assumed instantaneous nucleation conditions and we postulated that (for this particular case) 
delayed repassivation was not important. However, in the general case, both assumptions are invalid, and hence it is necessary to use Equation (45) to derive the extreme value distribution function. These calculations can only be performed numerically, in the general case, and their use in deriving extreme value distribution functions will be illustrated in a subsequent paper.

Proceeding now with our analysis of the pitting of manganese steel in $\mathrm{CO}_{2}$ acidified seawater, we see that fitting the scale parameter, $\alpha$, and the central parameter, $h$, from Equations (29) and (30) to the experimental data for $t=336$ hours yields $\alpha=3.7 \mu \mathrm{m}$ and $h=50 \mu \mathrm{m}$. In accordance with Equation (52), we have $\beta=0.011 \mu \mathrm{m} / \mathrm{h}$ and $V+$ $\beta \operatorname{Ln}\left(0.5 S N_{0}\right)=0.15 \mu \mathrm{m} / \mathrm{h}$. Using these values for the parameters, we calculated EVDs for different times assuming that, even for $t_{6}=1176$ hours, pit repassivation is not important. Satisfactory agreement between the experimental and predicted data is observed (Figure III-5.1).

DFA allows us to explore the influence of repassivation on the time-dependencies of parameters $\alpha$ and $h$. If the distribution in propagation rate is sufficiently sharp $(V>>\beta)$, Laplace's distribution can be considered to be an approximation of the $\delta$ function. Under these conditions, for the case of instantaneous nucleation, we have from Equation (44):

$$
F(x, t)=N_{0} \int_{x / V}^{\infty} \exp (-\gamma x / v) \psi(v) d v \approx N_{0} \exp (-\gamma x / V) \int_{x / V}^{\infty} \psi(v) d v
$$

By analogy with Equation (52), it is easily shown that parameters $\alpha$ and $h$ can be expressed as

$$
\alpha=\frac{\beta t}{1+\gamma \beta t / V} \text {, and } h=\frac{\left[V+\beta \operatorname{Ln}\left(0.5 S N_{0}\right)\right] t}{1+\gamma \beta t / V}
$$

We see that, for small values of $t$, the scale and central parameters can be described by Equation (52), i.e. they are proportional to time. However, for large observation times, parameters $\alpha$ and $h$ go to limits that are independent of time. This fact can be regarded 
as being physically evident, because at sufficiently large times all pits become passive (the damage function becomes "frozen") and further propagation of damage cannot occur, provided that $\gamma>0$. This conclusion concerning the achievement of limiting pitting depth also flows from a formal statistical treatment of the experimental data for underground pipelines made from carbon steels [4]. The "freezing" of damage functions can also be seen in the propagation of corrosion damage on aluminum in tap water [9].

In the more general case, we suggest the following, simple expressions for representing the time dependencies of the scale and central parameters

$$
\alpha=\frac{\alpha_{l} t}{1+\left(\alpha_{1} / \alpha_{\infty}\right) t} \text {, and } h=\frac{h_{l} t}{1+\left(h_{l} / h_{\infty}\right) t}
$$

For small times, Equations (55) yields the linear dependencies, $\alpha=\alpha_{1} t$ and $h=h_{1} t$, and for large times it yields the limiting values of $\alpha=\alpha_{\infty}$ and $h=h_{\infty}$. It is evident that experimental data for at least two times are required for determining the unknown parameters $\alpha_{l}, h_{1,} \alpha_{\infty}$ and $h_{\infty}$. Figure III-5.2 shows that Equations (55) can be used for extrapolating experimental data from Ref. 9 for the pitting of aluminum in tap water to the future. It is interesting to note that Finley [26] suggested that the experimental data for the pitting corrosion of aluminum in tap water (from Ref. 9) could be described by assuming that $\alpha$ does not change with time and that $h$ could be described by the logarithmic relation

$$
h=b \operatorname{Ln}(t)+c
$$

However, it is evident that Equation (56) cannot describe the experimental data for small values of $t$, when the condition $h \rightarrow 0$ for $t \rightarrow 0$ must be fulfilled, and for large values of $t$ when $h$ must go to a constant value. It should be emphasized that the power law (see Ref. 3) cannot describe the cessation of localized corrosion damage due to pit passivation. That is why, in our opinion, it is much more appropriate to use a hyperbolic law than to employ a logarithmic law (and power law) for representing the time dependencies of the EVS parameters. 




Figure III-5.2 Extreme value distribution functions (EVDs) versus exposure time for the pitting of manganese steel in CO2-acidified seawater. Only the data for $\mathrm{t}=336$ hours were used for fitting, in order to determine the central and scale parameters. Experimental data are taken from Figure 5 in Ref. 7.

It is important to note that the Gumbel asymptotic extreme (Type I) can be used for describing the statistics of localized corrosion damage when repassivation (death) is considered, even though nucleation is instantaneous (Figure III-5.3) or even for the case of progressive nucleation where two different nucleation functions have been considered (Figures III-5.4, III-5.5). Note that Figure III-5.4 shows the EVD for the nucleation rate function for the deterministic Point Defect Model. Numerical calculation shows that, in all of these cases, the dependencies of $\operatorname{Ln}(1-C D F)$ on pit depth, $x$, can be approximated by straight lines, at least for sufficiently large values of $x$. The work presented in this 
paper, therefore, provides a theoretical basis for applying Type I extreme value distributions for real corrosion systems.

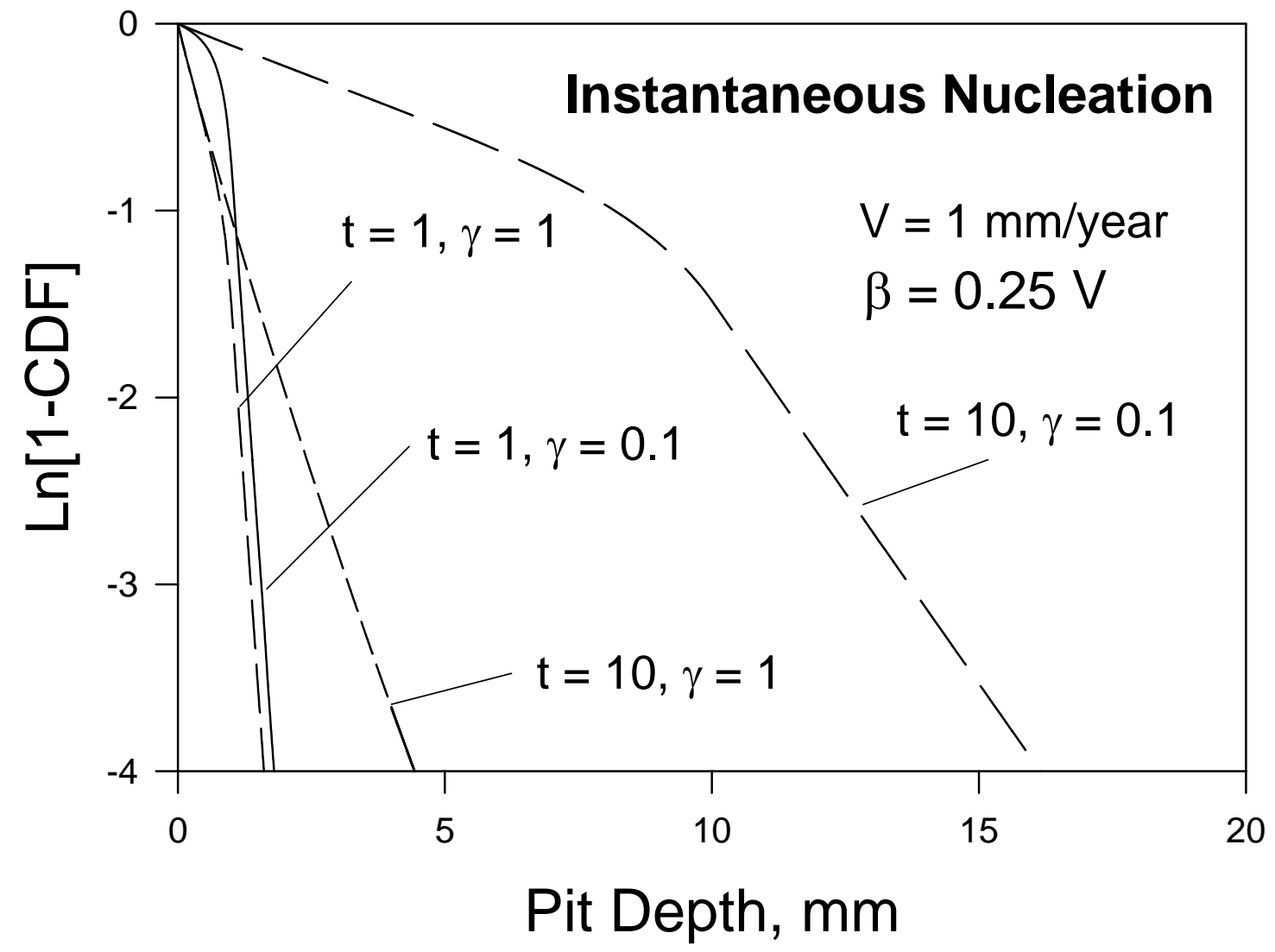

Figure III-5.3 Variation of the cumulative distribution function (CDF) with pit depth as a function of time for instantaneous nucleation. Observation times, $t$, are measured in years and the delayed repassivation constant, $\gamma$, is given in units of year [1]. 




Figure III-5.4 Variation of the cumulative distribution function (CDF) with pit depth as a function of time for the case when the nucleation of pits is described by the Pont Defect Model. Observation times, $t$, are measured in years and the delayed repassivation constant, $\gamma$, is given in units of year [1]. 


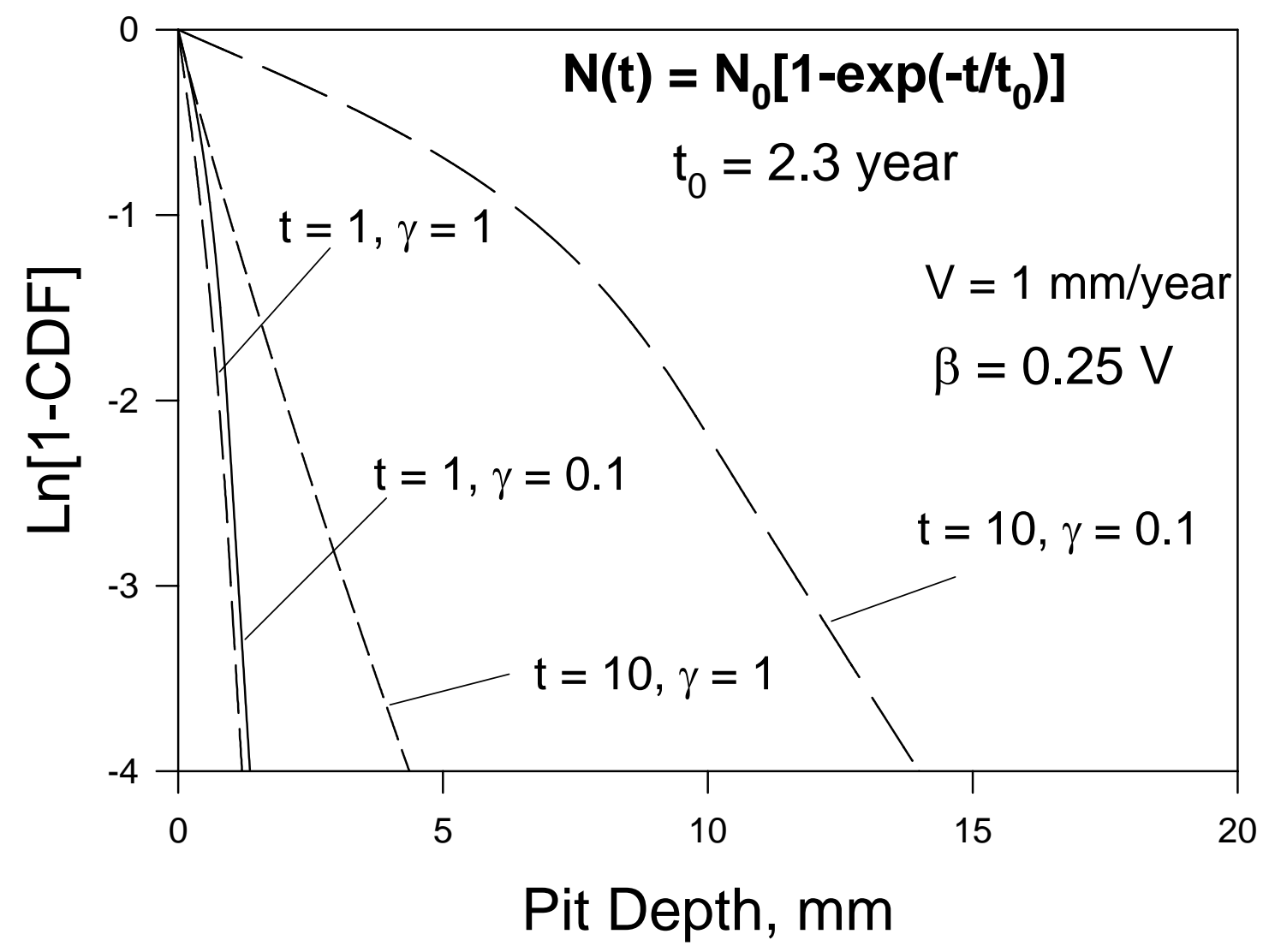

Figure III-5.5 Variation of the cumulative distribution function (CDF) with pit depth as a function of time for the case when the nucleation of pits is described by an exponential function. Observation times, $t$, are measured in years and the delayed repassivation constant, $\gamma$, is given in units of year [1].

\section{Issues and Concerns: $\quad$ None}

\section{III-5 References}

1. D. D. Macdonald and M. Urquidi-Macdonald, Corrosion, 48, 354 (1992).

2. D. D. Macdonald, C. Liu, M. Urquidi-Macdonald, G. H. Stickford, B. Hindin, A. K. Agrawal, and K. Krist, Corrosion, 50, 761 (1994).

3. P. J. Laycock, R. A. Cottis and P.A. Scarf, J. Electrochem. Soc., 137, 64 (1990).

4. L. I. Freiman. Progress in Science and Technology. Corrosion and Corrosion Protection [in Russian], 11, VINITI, p. 3, (1985).

5. G. Engelhardt, and D. D. Macdonald, Corrosion, 54, 469 (1998). 
6. G. Engelhardt and D. D. Macdonald. "The Deterministic Prediction of Localized Corrosion Damage in Aircraft Aluminum Alloys," in 1998 USAF Aircraft Structural Integrity Program Conference, 1- 3 December 1998, San Antonio, Texas.

7. J. E. Strutt, J. R. Nicholls and B. Barbier, Corros. Sci., 25, 305 (1985).

8. G. P. Marsh, I.D. Bland and K. J. Tailor, Br. Corr. J., 23, 157 (1988).

9. P. M. Aziz, Corrosion, 12, 495 (1956).

10. P.S. Pistorius and G.T. Burstein, Phil. Trans. R. Soc. Lond. A, 341, 531 (1992).

11. J. Stewart and D. Williams. "Studies of the initiation of pitting corrosion on stainless steels", in Advances in Localized Corrosion (ed. H. S. Isaacs et al.) NACE-International, Houston, Texas, pp.131-136 (1990).

12. D. Williams, J Stewart and P. H. Balkwill, Corr. Sci. 36, 1213 (1994).

13. L. F. Lin, C. Y. Chao, and D. D. Macdonald, J. Electrochem. Soc., 128, 1194 (1981).

14. D. D. Macdonald and M. Urquidi-Macdonald, Electrochim. Acta, 31, 1079 (1986).

15. D. D. Macdonald and M. Urquidi-Macdonald, J. Electrochem. Soc., 134, 41 (1987).

16. D. D. Macdonald and M. Urquidi-Macdonald, J. Electrochem. Soc., 136, 961 (1989).

17. D. D. Macdonald and M. Urquidi-Macdonald, J. Electrochem. Soc., 139, 3434 (1992).

13. L. F. Lin, C. Y. Chao, and D. D. Macdonald, J. Electrochem. Soc., 128, 1194 (1981).

14. D. D. Macdonald and M. Urquidi-Macdonald, Electrochim. Acta, 31, 1079 (1986).

15. D. D. Macdonald and M. Urquidi-Macdonald, J. Electrochem. Soc., 134, 41 (1987).

16. D. D. Macdonald and M. Urquidi-Macdonald, J. Electrochem. Soc., 136, 961 (1989).

17. D. D. Macdonald and M. Urquidi-Macdonald, J. Electrochem. Soc., 139, 3434 (1992).

18. Z. Szklarska-Smialowska, Pitting Corrosion of Metals, NACE-International, Houston, 1986.

19. G. R. Engelhardt, D. D. Macdonald, and M. Urquidi-Macdonald, Corros. Sci., 41, 2267 (1999).

20. G. Engelhardt and D. D. Macdonald, Corr. Sci., (2004). In press.

21. G. E. Eldredge, Corrosion, 13, 51 (1957)

22. E. J. Gumbel, Statistics of Extremes, Columbia University Press, NY, (1957).

23. A. Turnbull, Br. Corros. J., 28, 297 (1993).

24. T Shibata et al., Corros. Eng., 37, 699 (1988).

25. A. Korn and M. Korn, "Mathematical Handbook", McGraw-Hill, NY, (1968). 
26. H. F. Finley, Corrosion, 23, 83 (1967).

27. T. Haruna and D. D. Macdonald, J. Electrochem. Soc., 144, 1574 (1997).

28. I. Fonseca, N. Lima, J. A. Rodrigues, M. I. S. Pereira, J. C. S. Salvador, M. G. S. Ferreira, Electrochemistry Communications, 4, 353 (2002).

29. Handbook of Physics and Chemistry, (Edit. R. C. Weast), CRC Press, Boca Raton, Fl, $59^{\text {th }}$ Edit., 1978/79.

30. D. Ellerbrock and D. D. Macdonald, Mater. Sci. Forum, 185-188, 927 (1995).

31. S. Ahn, H-S. Kwon, and D. D. Macdonald, "The Role of Chloride Ion in Passivity Breakdown on Nickel", Proc. Symp. Surface Oxide Films, Electrochemical Society, Orlando, FL, Oct. 12-17, 2004, in press.

32. T. Shibata, Trans ISIJ, 23, 785 (1983).

33. R. Fratesi, Corrosion, 41, 114 (1985).

\section{IV.Publications in Peer Reviewed Journals and Conference Proceedings}

1. J. Liu and D. D. Macdonald, "The Passivity of Iron in the Presence of Ethylenediaminetetraacetic Acid II. The Defect and Electronic Structures of the Barrier Layer", J. Electrochem. Soc., 148 (11), B425-B430 (2001)

2. Vankeerberghen, M., and D. D. Macdonald, "Predicting Crack Growth Rate vs. Temperature-Behaviour of Type 304 Stainless Steel in Dilute Sulfuric Acid Solutions", Corros. Sci., 44, 1425-1441 (2002).

3. Macdonald, D. D., et.al., "High temperature on-line monitoring of water chemistry and corrosion control in water cooled power reactors; Report of a coordinated research project 1995-1999", Proc. IAEA-TECDOC-1303, International Atomic Energy Agency (IAEA), Vienna, Austria, July 2002.

4. Liu, S. and D. D. Macdonald, "Fracture of AISI 4340 Steel in Concentrated Sodium Hydroxide Solution", Corrosion, 58, 835-845 (2002).

5. Sikora, E. and D. D. Macdonald, "Nature of the Passive Film on Nickel", Electrochimica Acta, 48, 69-77 (2002).

6. Macdonald, D. D., "Comments on 'Electrochemical Transients During the Initial Moments of Anodic Oxidation of Aluminum' by Huiguan Wu and Kurt Hebert, Electrochimica Acta, 47, 1373-1383 (2002)", Electrochimica Acta, 48, 127-130 (2002)

7. Macdonald, D. D., "Controlling IGSCC in BWR Coolant Circuits", Proc. 2nd ${ }^{\text {st }}$ DAWAC Conf., Int. Atomic Energy Agency, Prague, Czech Republic, Nov. 26-29, (2002) 
8. Macdonald, D. D. and G. R. Engelhardt, "Deterministic Prediction of Localized Corrosion Damage - A Reflective Review of Critical Issues", J. Corros. Sci. Engin., 6, Paper C066 (2003).

9. Macdonald, D. D., "Internal/External Environment Coupling in Stress Corrosion Cracking”, J. Corros. Sci. Engin., 6, Paper C065 (2003).

10. Vankeerberghen, M. and D. D. Macdonald, "Calculating the Temperature Maximum and the Lower Potential Limit for the Crack Growth Rate in Type 304 SS Using the CEFM", Paper 03250, CORROSION2003, San Diego, CA, March 2003.

11. Macdonald, D. D. and Gomez-Duran, M., "Stress Corrosion Cracking of Sensitized Type 304 Stainless Steel in Thiosulfate Solution: I. Fate of the Coupling Current", Corrosion Science, 45, 1455-1471 (2003)

12. Englehardt, G. and D. D. Macdonald, "Estimation of Corrosion Cavity Growth Rate for Predicting System Service Life”, Corrosion Science, 46(5), 1159-1187 (2004).

13. Englehardt, G. and D. D. Macdonald, "Unification of the Deterministic and Statistical Approaches for Predicting Localized Corrosion Damage: I. Theoretical Foundation", Corrosion Science, in press (2003).

14. Macdonald, D. D., "Passive Films: Nature's Exquisitely Nano-Engineered Protection System", Current Applied Physics, 4(2-4), 129-132 (2004).

15. Macdonald, D. D. and G. Englehardt, "Theory For The Deterministic Prediction Of Pitting Corrosion Damage On Aluminum", Proc. Electrochemical Society, Orlando, FL, Oct. 12-17, 2003.

16. Ahn, S., HyukSang Kwon, and D. D. Macdonald, "The Role of Chloride Ion in Passivity Breakdown on Nickel", Proc. Electrochemical Society, Orlando, FL, Oct. 12-17, 2003.

17. Gomez-Duran, M. and Macdonald, D. D., "Stress Corrosion Cracking of Sensitized Type 304 Stainless Steel in Thiosulfate Solution: II. Dynamics of Fracture", Corrosion Science, submitted 2004.

18. Macdonald, D. D., "Critical Issues in Understanding Corrosion and Electrochemical Phenomena in Super Critical Aqueous Media”, Paper \#04484, CORROSION '04, New Orleans, LA, March 2004.

19. Macdonald, D. D., "After Two Decades of Predicting Crack Growth Rate - What Have We Learned?", Paper \#04673, CORROSION '04, New Orleans, LA, March 2004.

20. Soundararajan, B. and Macdonald, D. D., "Caustic Cracking of AISI 4340 Steel", Paper \#04572, CORROSION '04, New Orleans, LA, March 2004.

21. Macdonald, D. D., "Fate of the Coupling Current in Stress Corrosion Cracking Mechanistic and Corrosion Control Implications", Paper \#04570, CORROSION '04, New Orleans, LA, March 2004. 


\section{Presentations}

1. D. D. Macdonald, "The Holy Grail: Deterministic Prediction of Corrosion Damage Thousands of Years into the Future", International Workshop on the Prediction of Long Term Corrosion Behavior in Nuclear Waste Systems", Chateau de Cadarache, France, Nov. 26-29, 2001.

2. D. D. Macdonald, "A Brief History of Electrochemical Impedance Spectroscopy", Centenary Meeting of the Electrochemical Society, Philadelphia, PA, May 12-17, 2002. Invited.

3. D. D. Macdonald, "Recent Advances in the Theory of Stress Corrosion Cracking", Institute of Nuclear Safety Systems, Osaka, Japan, May 26-30, 2002. Invited.

4. D. D. Macdonald, "Passivity, the Enabler of Our Metals-Based Civilization", University of Florida, July 12, 2002. Invited.

5. D. D. Macdonald, "Passivity and Passivity Breakdown", Georgia Institute of Technology, July 12, 2002. Invited.

6. D. D. Macdonald, "Can We Predict the Future in Corrosion Science?", 2002 DOE Corrosion Contractors Meeting, Brookhaven National Laboratory, September 1314, 2002.

7. D. D. Macdonald, "Can Corrosion Science Predict the Future?", presented in receipt of the U. R. Evans Award, British Corrosion Institute, Cardiff, Wales, October 23, 2002. Invited.

8. D. D. Macdonald, "Passive Films, Nature's Exquisitely Nano-Engineered Protection System", AMN-1, The Macdiarmid Institute for Advanced Materials and Nano Technology, Tepapa, Wellington, New Zealand, February 13, 2003. Invited.

9. M. Vankeerberghen and D. D. Macdonald, "Calculating the TemperatureMaximum and the Lower Potential Limit for the Crack Growth Rate in Type 304 SS Using the CEFM", CORROSION 2003, (NACE International, Houston, TX), San Diego, CA March 17-27, 2003.

10. D. D. Macdonald, "Mixed Potential-Point Defect Models for Nuclear Waste Containers", CORROSION 2003, (NACE International, Houston, TX), San Diego, CA March 17-27, 2003. Invited.

11. D. D. Macdonald, J. Liu, B. Marx, B. Soundararajan, M. Smith, and S. Ahn, "Development of Advanced Electrochemical Emission Spectroscopy for Monitoring Corrosion in Simulated DOE Liquid Waste”, EMSP Worksop, Pacific Northwest National Laboratory, Richland, WA, May 6-8, 2003. Invited. 
12. G. Engelhardt and D. D. Macdonald, "Prediction of the Accumulation of Localized Corrosion Damage to Low Pressure Steam turbines in Fossil Service", Seventh Intl. Conf. Cycle Chem. Fossil Plants, Houston, TX, June 3-5, 2003. Invited.

13. D. D. Macdonald, "Deterministic Prediction of Localized Corrosion", Intl. Symp. Corrosion Science in the $21^{\text {st }}$ Century, UMIST, Manchester, UK, July 6-11, 2003.

14. D. D. Macdonald, "Internal/External Environment Coupling in Stress Corrosion Cracking", Intl. Symp. Corrosion Science in the $21^{\text {st }}$ Century, UMIST, Manchester, UK, July 6-11, 2003. (Keynote Lecture).

15. D. D. Macdonald and G. R. Engelhardt, "Prediction of Long-Term Corrosion Damage in High Level Nuclear Waste Disposal Systems", Corrosion Workshop on Yucca Mountain Waste Repository", Department of Energy, Bethesda, MD, July 28-30, 2003. Invited.

16. D. D. Macdonald, G. R. Engelhardt, and Y. Zhang, "Prediction of Localized Corrosion Damage in Low Pressure Steam Turbines", EPRI, Charlotte, NC, August 11, 2003. Invited.

VI. Current Student Status

\begin{tabular}{|c|c|c|c|c|}
\hline Student Name & $\begin{array}{c}\text { Date } \\
\text { Started }\end{array}$ & Degree & $\begin{array}{c}\text { Candidacy } \\
\text { Status }\end{array}$ & $\begin{array}{c}\text { Expected } \\
\text { Graduation }\end{array}$ \\
\hline Brian M. Marx & $8 / 2002$ & M.S./Ph.D. & Passed-9/2003 & $8 / 2006$ \\
\hline Balaji Soundararajin & $8 / 2002$ & M.S./Ph.D & Taking-9/2004 & $8 / 2006$ \\
\hline Morgan Smith & $1 / 2003$ & M.S. & N/A & $5 / 2005$ \\
\hline
\end{tabular}

